\title{
CfA3: 185 TYPE la SUPERNOVA LIGHT CURVES FROM THE CfA
}

\section{Citation}

Hicken, Malcolm, Peter Challis, Saurabh Jha, Robert P. Kirshner, Tom Matheson, Maryam Modjaz, Armin Rest, et al. 2009. “CfA3: 185 TYPE la SUPERNOVA LIGHT CURVES FROM THE CfA." The Astrophysical Journal 700 (1): 331-57. https://doi.org/10.1088/0004-637x/700/1/331.

\section{Permanent link}

http://nrs.harvard.edu/urn-3:HUL.InstRepos:41399738

\section{Terms of Use}

This article was downloaded from Harvard University's DASH repository, and is made available under the terms and conditions applicable to Other Posted Material, as set forth at http:// nrs.harvard.edu/urn-3:HUL.InstRepos:dash.current.terms-of-use\#LAA

\section{Share Your Story}

The Harvard community has made this article openly available.

Please share how this access benefits you. Submit a story.

Accessibility 


\title{
CfA3: 185 TYPE Ia SUPERNOVA LIGHT CURVES FROM THE CfA
}

\author{
Malcolm Hicken $^{1,2}$, Peter Challis ${ }^{1}$, Saurabh Jha $^{3}$, Robert P. Kirshner ${ }^{1}$, Tom Matheson ${ }^{4}$, Maryam Modjaz $^{5}$, \\ Armin Rest $^{2}$, W. Michael Wood-Vasey ${ }^{6}$, Gaspar Bakos ${ }^{1}$, Elizabeth J. Barton ${ }^{7}$, Perry Berlind ${ }^{1}$, Ann BragG ${ }^{8}$, \\ Cesar Briceño ${ }^{9}$, Warren R. Brown ${ }^{1}$, Nelson Caldwell ${ }^{1}$, Mike Calkins $^{1}$, Richard Cho ${ }^{1}$, Larry Ciupik ${ }^{10}$, \\ Maria Contreras $^{1}$, Kristi-Concannon Dendy ${ }^{11}$, Anil Dosaj ${ }^{1}$, Nick Durham ${ }^{1}$, Kris Eriksen ${ }^{12}$, Gil Esquerdo $^{13}$, \\ Mark Everett $^{13}$, Emilio Falco ${ }^{1}$, Jose Fernandez ${ }^{1}$, Alejandro Gaba ${ }^{11}$, Peter Garnavich ${ }^{14}$, Genevieve Graves ${ }^{15}$, \\ Paul Green $^{1}{ }$, Ted Groner ${ }^{1}$, Carl Hergenrother ${ }^{12}$, Matthew J. Holman ${ }^{1}$, Vit Hradecky ${ }^{1}$, John Huchra ${ }^{1}$, \\ Bob Hutchison ${ }^{1}$, Diab Jerius ${ }^{1}$, Andres Jordan ${ }^{1}$, Roy Kilgard ${ }^{1}$, Miriam Krauss ${ }^{16}$, Kevin Luhman ${ }^{17}$, Lucas Macri ${ }^{18}$, \\ Daniel Marrone ${ }^{1}$, Jonathan McDowell ${ }^{1}$, Daniel McIntosh ${ }^{19}$, Brian McNamara $^{20}$, Tom Megeath ${ }^{1}$, \\ Barbara Mochejska ${ }^{21}$, Diego Munoz ${ }^{1}$, James Muzerolle ${ }^{12}$, Orlando Naranjo ${ }^{1}$, Gautham Narayan ${ }^{1}$, Michael Pahre ${ }^{1}$, \\ Wayne Peters ${ }^{1}$, Dawn Peterson $^{1}$, Ken Rines $^{1}$, Ben Ripman ${ }^{1}$, Anna Roussanova ${ }^{16}$, Rudolph Schild ${ }^{1}$, \\ Aurora Sicilia-Aguilar ${ }^{1}$, Jennifer Sokoloski ${ }^{1}$, Kyle Smalley ${ }^{1}$, Andy Smith $^{1}$, Tim Spahr ${ }^{1}$, K. Z. StaneK ${ }^{22}$, \\ Pauline Barmby ${ }^{23}$, Stéphane Blondin ${ }^{24}$, Christopher W. Stubbs ${ }^{1,2}$, Andrew Szentgyorgit ${ }^{1}$, Manuel A. P. Torres ${ }^{1}$, \\ Amili VaZ ${ }^{16}$, Alexey Vikhlinin ${ }^{1}$, Zhong Wang $^{1}$, Mike Westover ${ }^{1}$, Deborah Woods ${ }^{1}$, and Ping Zhao ${ }^{1}$ \\ ${ }^{1}$ Harvard-Smithsonian Center for Astrophysics, Cambridge, MA 02138, USA; mhicken@cfa.harvard.edu, kirshner@cfa.harvard.edu \\ ${ }^{2}$ Department of Physics, Harvard University, Cambridge, MA 02138, USA \\ ${ }^{3}$ Department of Physics and Astronomy, Rutgers, the State University of New Jersey, Piscataway, NJ 08854, USA \\ ${ }^{4}$ National Optical Astronomy Observatory, NOAO Gemini Science Center, 950 North, Cherry Avenue, Tucson, AZ 85719, USA \\ 5 Astronomy Department, UC Berkeley, Campbell Hall, Berkeley, CA 94720, USA \\ ${ }^{6}$ Department of Physics and Astronomy, University of Pittsburgh, Pittsburgh, PA 15260, USA \\ ${ }^{7}$ Department of Physics and Astronomy, University of California, 2158 Frederick Reines Hall, Irvine, CA 92697, USA \\ ${ }^{8}$ Physics Department, Marietta College, 215 Firth Street, Marietta, OH 45750, USA \\ ${ }^{9}$ Centro de Investigaciones de Astronomia (CIDA), Apdo Postal 264 VE, Merida 5101-A, Bolivarian Republic of Venezuela \\ ${ }^{10}$ Adler Planetarium \& Astronomy Museum, 1300 S. Lake Shore Drive, Chicago, IL 60605, USA \\ ${ }^{11}$ Department of Physics and Astronomy, University of North Carolina at Chapel Hill, NC 27599-3255, USA \\ 12 The University of Arizona, Tucson, AZ 85721, USA \\ ${ }^{13}$ Planetary Science Institute, 1700 E. Fort Lowell Road, Tucson, AZ 85719, USA \\ ${ }^{14}$ University of Notre Dame, 25 Nieuwland Science Hall, Notre Dame, IN 46556-5670, USA \\ ${ }^{15}$ University of California, Astronomy \& Astrophysics, 201 Interdisciplinary Sciences Building (ISB), Santa Cruz, CA 95064, USA \\ ${ }^{16}$ Massachusetts Institute of Technology, 77 Massachusetts Avenue, Cambridge, MA 02139-4307, USA \\ ${ }^{17}$ Department of Astronomy and Astrophysics, 525 Davey Lab, The Pennsylvania State University, University Park, PA 16802, USA \\ ${ }^{18}$ George P. and Cynthia W. Mitchell Institute for Fundamental Physics and Astronomy, Department of Physics, Texas A\&M University, 4242 TAMU, \\ College Station, TX 77843-4242, USA \\ ${ }^{19}$ Department of Astronomy, University of Massachusetts, Amherst, MA 01003, USA \\ ${ }^{20}$ Department of Physics \& Astronomy, University of Waterloo, 200 University Avenue, West Waterloo, Ontario N2L 3G1, Canada \\ ${ }^{21}$ Copernicus Astronomical Center in Warsaw, Poland Bartycka 18, 00-716 Warszawa, Poland \\ ${ }^{22}$ OSU Department of Astronomy, McPherson Laboratory $140 \mathrm{~W} 18$ th Avenue, Columbus, OH 43210, USA \\ ${ }^{23}$ Department of Physics \& Astronomy, University of Western Ontario, London, ON N6A 3K7, Canada \\ ${ }^{24}$ European Southern Observatory, D-85748 Garching, Germany \\ Received 2009 February 5; accepted 2009 May 20; published 2009 July 1
}

\begin{abstract}
We present multiband photometry of 185 type-Ia supernovae (SNe Ia), with over 11,500 observations. These were acquired between 2001 and 2008 at the F. L. Whipple Observatory of the Harvard-Smithsonian Center for Astrophysics (CfA). This sample contains the largest number of homogeneously observed and reduced nearby $\mathrm{SNe}$ Ia $(z \lesssim 0.08)$ published to date. It more than doubles the nearby sample, bringing SN Ia cosmology to the point where systematic uncertainties dominate. Our natural system photometry has a precision of $\lesssim 0.02 \mathrm{mag}$ in $B V R I r^{\prime} i^{\prime}$ and $\lesssim 0.04 \mathrm{mag}$ in $U$ for points brighter than $17.5 \mathrm{mag}$. We also estimate a systematic uncertainty of $0.03 \mathrm{mag}$ in our SN Ia standard system $B V R I r^{\prime} i^{\prime}$ photometry and 0.07 mag for $U$. Comparisons of our standard system photometry with published SN Ia light curves and comparison stars, where available for the same SN, reveal agreement at the level of a few hundredths mag in most cases. We find that 1991bg-like SNe Ia are sufficiently distinct from other SNe Ia in their color and light-curve-shape/ luminosity relation that they should be treated separately in light-curve/distance fitter training samples. The CfA3 sample will contribute to the development of better light-curve/distance fitters, particularly in the few dozen cases where near-infrared photometry has been obtained and, together, can help disentangle host-galaxy reddening from intrinsic supernova color, reducing the systematic uncertainty in SN Ia distances due to dust.
\end{abstract}

Key words: supernovae: general

Online-only material: color figures, machine-readable tables 


\section{INTRODUCTION}

SNe Ia are standardizable candles $(\sigma \lesssim 0.2 \mathrm{mag}$ after correction for light-curve shape) and have been used to measure the expansion history of the universe (e.g., Phillips 1993; Riess et al. 1996; Hamuy et al. 1996a; Goldhaber et al. 2001; Jha et al. 2007), giving rise to the startling conclusion that the universe is accelerating (e.g., Riess et al. 1998; Perlmutter et al. 1999).

Some 30 years prior to the discovery of the accelerating universe, Kowal (1968) found a dispersion of $\sim 0.6 \mathrm{mag}$ in the SN Ia redshift-magnitude relation. Reasons for the high dispersion are that many of the objects in his sample were not in the Hubble flow, corrections for light-curve shape and absorption were not made, and not all objects were SNe Ia. $\mathrm{He}$ predicted that $\mathrm{SNe}$ Ia might become distance estimators with better than $10 \%$ precision and enable measurement of the curvature of the Hubble diagram at greater redshifts. Nearly 20 years later, Norgaard-Nielsen et al. (1989) made a valiant attempt to measure $\mathrm{SNe}$ Ia at $z \sim 0.3$, using methods similar to modern SN searches. They had the right idea but their telescope and detector were too small and they only reported one SN Ia in two years of searching. The advent of significantly larger detectors, mounted on larger telescopes, provided the higher discovery rates needed and was one of the main factors in the discovery of the accelerating universe.

SN Ia studies can be divided into two broad groups: low redshift and high redshift. For our purposes, the dividing line between the two groups is at $z \approx 0.15$. Low-redshift SNe Ia are easier to study to higher precision and can generally be observed over a greater range in phase. They map out the recent expansion of the universe and can be used to study the local bulk flows and peculiar velocities of galaxies in the nearby universe (e.g., Tammann \& Leibundgut 1990; Riess et al. 1995; Jha et al. 2007; Neill et al. 2007; Haugbolle et al. 2007). They also serve as the template against which the high-redshift $\mathrm{SNe}$ Ia are compared. Having, and understanding, a nearby sample that fills out the phase space of SN Ia properties is vital to the use of SNe Ia as precise distance indicators at greater redshifts. High-redshift $\mathrm{SNe}$ Ia allow measurements of the change in the expansion rate of the universe over time as well as in any presumed underlying models, such as dark energy. Up to a point, more data at both high and low redshift decrease the statistical uncertainty in the derived cosmological parameters. It can also help refine our understanding of possible systematic uncertainties such as hostgalaxy reddening and intrinsic color variation of SNe Ia.

On the nearby front, the Calan-Tololo survey produced the first large, multiband, CCD sample of SN Ia photometry, publishing 29 light curves (Hamuy et al. 1996b). This was followed by 22 BVRI SN Ia light curves from the CfA in 1999 (Riess et al. 1999) and a further 44 UBVRI in 2006 (Jha et al. 2006, hereafter, J06) (these two samples will be referred to as CfA1 and CfA2, respectively). Additionally, Krisciunas and his collaborators have published a significant number (Krisciunas et al. 2000, 2001, 2003, 2004a, 2004b, 2006), the European Supernova Collaboration has published photometry of several nearby SNe Ia (see Stanishev et al. 2007, and references therein), and Kowalski et al. (2008) recently published eight nearby SNe Ia.

Other groups that are working on significant nearby samples are KAIT, ${ }^{25}$ in conjunction with the LOTOSS/LOSS SN searches, CSP (Hamuy et al. 2006) and the Nearby Supernova

\footnotetext{
25 http://astro.berkeley.edu/ bait/kait.html
}

Factory (Aldering et al. 2002). The 2004 Sloan Digital Sky Survey (SDSS) SN Survey (Sako et al. 2005) found 16 spectroscopically confirmed SNe Ia as a preparation run for the SDSS-II SN Survey (Frieman et al. 2008). In its first two years, SDSS-II observed over 300 spectroscopically confirmed and $\sim 100$ photometrically identified SNe Ia in ugriz and in the redshift range $0.05<z<0.35$. This survey has good control of systematics in their photometry and will be very useful in calculating SN rates in the nearby to intermediate redshift range. Dilday et al. (2008) present a calculation of nearby SN Ia rates based on 17 SNe Ia at $z \leqslant 0.12$ from the 2005 season of SDSS-II.

Systematic differences often exist between different groups' photometry of the same SN Ia at low redshift, typically at the level of a few hundredths mag and sometimes larger. These differences are mainly due to difficulties in transforming to the standard system and, to a lesser extent, the use of different photometry pipelines. A large, homogeneously observed and reduced nearby sample does not internally suffer from these two problems and can help reduce systematic uncertainties in dark energy measurements. However, there is still the issue of ensuring that the nearby and faraway samples are photometrically consistent.

The goal of our research was to produce a large sample of homogeneously observed and reduced SN Ia light curves that fills out the sampling of the whole range of SN Ia properties and can be used to reduce the statistical and systematic uncertainties in SN Ia cosmology. Here, we publish 185 multiband optical SN Ia light curves, with data taken between the years 2001 and 2008. This is the third sample of nearby CfA SN Ia photometry (CfA3 sample). This is the largest set of nearby SN Ia photometry to date, more than doubling the literature sample. It consists of over 11,500 observations. For comparison, the CfA1 and CfA2 samples consist of 1210 and 2190 observations.

A better understanding of the nature and range of SN Ia properties improves their use as standardizable candles and may reveal that certain subsamples are more useful than others. We intentionally built up the sample of slow (more luminous) and fast (less luminous) decliners. The slow decliners are particularly helpful for improving SN Ia cosmology since they are found more often at high redshift. The study of peculiar SNe Ia also deepens our understanding of what physical mechanisms might be at work and large samples are more likely to include rare types. One such object that the CfA Supernova Group ${ }^{26}$ observed was SN 2006gz (Hicken et al. 2007; Maeda et al. 2009), a very slow-declining and bright SN Ia that may have come from a double-degenerate merger and/or a Super-Chandrasekhar progenitor. With the larger sample, light-curve fitters can be trained better and a proper prediction error can be calculated by excluding individual objects (or groups of objects) from the training sample one at a time. Mandel et al. (2009) has developed the machinery for this in the near infrared and will be including the optical bands shortly. Combining the optical and near-infrared photometry should help disentangle host-galaxy reddening from intrinsic SN Ia color.

The impact of adding additional nearby SNe Ia can be seen in Kowalski et al. (2008), who take 49 nearby and 250 faraway $\mathrm{SNe}$ Ia from the literature and add eight of their own, using the light-curve fitter SALT (Guy et al. 2005). These additional eight reduce the statistical uncertainty on the dark energy parameter, $w$, by a factor of 1.04 , when the intrinsic or additional uncertainty of $\sim 0.1 \mathrm{mag}$ that they discuss is added. If the

\footnotetext{
26 http://www.cfa.harvard.edu/supernova/
} 
intrinsic uncertainty is not added then their eight reduce the statistical uncertainty in $w$ by a factor of 1.07. The application of the CfA3 data set to studying dark energy is presented in Hicken et al. (2009, hereafter H09), where 90 of the 185 objects presented here pass the quality cuts (on such things as redshift and phase of first observation) of Kowalski et al. (2008). These 90 are added to their "Union" set to form the "Constitution" set (a more perfect union) with a total of 157 nearby and 250 faraway objects. The Constitution set produces a value of $1+w=0.013_{-0.068}^{+0.066}(0.11$ syst $)$, consistent with the cosmological constant. The uncertainty on $w$ for the Constitution set is found to be 1.2-1.3 times smaller than the comparable Union value (1.3 when the $\sim 0.1 \mathrm{mag}$ intrinsic uncertainty is included and 1.2 when it is not), in line with approximate statistical expectations. The systematic uncertainty is estimated to be $\sim 65 \%$ larger than the statistical uncertainty. The other fitters, SALT2 (Guy et al. 2007), MLCS2k2 (Jha et al. 2007) with $R_{V}=3.1$ and MLCS2k2 with $R_{V}=1.7$ were found to reduce the statistical uncertainty by a factor of $\sim 1.2-1.3$, slightly less than the rough statistical expectation. The addition of the CfA 3 sample achieves its goal of reducing the statistical uncertainty of $w$. Both the good and the bad news is that systematic errors are now the main limit for making further progress in better understanding dark energy with SNe Ia! Improvements in systematic uncertainties are needed to maximize the contributions of future SN Ia surveys, such as the Joint Dark Energy Mission, that aim to place tight constraints on the time dependence of dark energy.

In this paper, we first show that the CfA3 sample is consistent with previous nearby samples in its color and host-galaxy reddening distributions. By design, the CfA3 sample has a wider distribution of light-curve shapes than earlier work since we gave the fast and slow decliners higher priority in deciding which objects to follow most thoroughly. However, the range of decline rates covered is the same. We also show that the agreement of our photometry with that of other groups, for the same objects, is as good as the agreement between other groups, typically at the level of a few hundredths mag. These facts give us confidence that the CfA3 sample can be used by current light-curve fitters developed primarily from the Calan-Tololo, CfA1, and CfA2 surveys. We invite people to combine the CfA3 sample with previous samples to retrain existing fitters or invent new ones. The CfA3 sample itself was not part of the training sample for any of the light-curve fitters in H09 and so the good agreement found there of the $\mathrm{CfA} 3$ results with previous samples is encouraging.

The CfA3 sample shares many of its methods with ESSENCE (Miknaitis et al. 2007). The same data-reduction pipeline was used, minimizing the introduction of systematic effects due to different reduction methods. The CfA3 sample also helps reduce the systematic uncertainty in $w$ because it was reduced and mostly observed in a homogeneous fashion (the use of two different cameras and changing from $R I$ filters to $r^{\prime} i^{\prime}$ being the exceptions to completely homogeneous observation).

In conjunction with this optical photometry, the CfA Supernova Group has taken spectra (Matheson et al. 2008; S. Blondin et al. 2009, in preparation) of many of these SNe using the FAST spectrograph (Fabricant et al. 1998) and, starting in 2004, began the use of the PAIRITEL near-infrared telescope ${ }^{27}$ to acquire valuable $J H K$-band data for the brighter SN Ia in the sample. These near-infrared SN Ia light curves stand on their own as standard candles (Wood-Vasey et al. 2008) and, when

\footnotetext{
27 http://www.pairitel.org/
}

combined with the optical data, will help clarify the properties of host-galaxy dust and intrinsic color variation of SNe Ia (A. Friedman et al. 2009, in preparation). This should help decrease the systematic uncertainties due to these intertwined phenomena.

In Section 2, we describe our observing strategy, explain our data reduction choices, and present the CfA3 light curves. We also show that our photometry is internally consistent and agrees well externally (to a few hundredths mag, roughly) in cases where others have published light curves for the same objects. Typical uncertainties in our $V$-band SN photometry are $0.015 \mathrm{mag}$ around maximum light. We estimate a systematic uncertainty of $0.03 \mathrm{mag}$ in $B V R I r^{\prime} i^{\prime}$ and $0.07 \mathrm{mag}$ in $U$. In Section 3, we examine the decline rates, intrinsic colors, and intrinsic absolute magnitudes. We confirm many of the relations seen before. The one main new insight is that the fast decliners have a range in intrinsic magnitude of 1.0-1.5 mag, with the 1991 bg-like objects significantly fainter and not forming part of the otherwise tight locus of SN Ia points, suggesting that they should be treated separately in light-curve fitter training samples. We present our conclusions in Section 4.

The CfA3 light curves, comparison star magnitudes, and passbands can be found at our Web $\operatorname{site}^{28}$ and are archived with the journal. Luminosity distances from H09 can also be found at our Web site.

\section{DATA AND REDUCTION}

The instruments, data acquisition, and data reduction are described here. The data reduction consists of three stages: reduction, calibration, and host-galaxy subtraction (where necessary). The reduction and subtraction stages are carried out by a version of the ESSENCE and SuperMACHO pipeline (Miknaitis et al. 2007; Rest et al. 2005; Garg et al. 2007), modified for use on the CfA3 data. The calibration was carried out very similarly to the calibration in the CfA1 and CfA2 samples. We made use of differential photometry by calibrating the field or comparison stars surrounding the SN on photometric nights and then measuring the flux of the $\mathrm{SN}$ relative to the comparison stars in each image, on both photometric and nonphotometric nights. In most cases, the underlying host-galaxy light had to be subtracted, using reference images taken after the $\mathrm{SN}$ had faded.

\subsection{Instruments}

The $1.2 \mathrm{~m}$ telescope at the F. L. Whipple Observatory (FLWO) was used to obtain the photometry presented here. The 4Shooter $2 \times 2$ CCD mosaic ${ }^{29}$ was employed up until 2004 August for 64 objects of the CfA3 sample. From 2004 September to 2005 July, the $2 \times 1 \mathrm{CCD}$ mosaic Minicam ${ }^{30}$ was used to observe five SNe Ia. The single-chip CCD Keplercam ${ }^{31}$ was used for the remaining $116 \mathrm{SNe}$ Ia beginning in 2005 September.

The 4Shooter camera uses four thinned, backsideilluminated, antireflective coated Loral $2048 \times 2048$ CCD detectors. Our 4Shooter observations were always on chip three (read out by a single amplifier) in the bin-by- 2 mode such that the binned pixel scale is $0^{\prime \prime} 674$ pixel $^{-1}$ and the field of view was $11^{\prime} .5 \times 11.5$. The typical image quality was $1^{\prime \prime} .5$ to $3^{\prime \prime}$ FWHM. The Minicam chips are thinned, backside-illuminated Marconi

\footnotetext{
28 http://www.cfa.harvard.edu/supernova/CfA3

29 http://linmax.sao.arizona.edu/FLWO/48/OLD/4shccd.html

30 http://linmax.sao.arizona.edu/FLWO/48/OLD/miniccd.html

$31 \mathrm{http}: / /$ linmax.sao.arizona.edu/FLWO/48/kepccd.html
} 
(ex-EEV) $2248 \times 4640$ CCD detectors with two long-rectangular shaped amplifiers per chip. In the bin-by-2 mode, the pixel scale is $0^{\prime \prime} 600 \mathrm{pixel}^{-1}$. Our observations were always on amplifier three with an approximate field of view of $5^{\prime} \cdot 1 \times 23^{\prime} \cdot 1$. The Keplercam uses a Fairchild "CCD 486." It is read out by four amplifiers, each covering a region of $2048 \times 2048$ pixels. Our observations were always on amplifier 2 . In the bin-by-2 mode, the pixel scale is 0.672 pixel $^{-1}$, resulting in an amplifier-2 field of view of approximately $11.5 \times 11^{\prime} .5$.

All three instruments have good response in the red while the 4Shooter was superior in the near ultraviolet. The 4Shooter had a significant number of bad pixels that required masking, the Minicam had few and the Keplercam had virtually none. A bad-pixel mask was not required or used for the Minicam and Keplercam. The Johnson $U B V$ passbands were used with all three detectors. The Krons-Cousins $R I$ passbands were used on the 4Shooter. In order to cooperate better with other FLWO observing programs, SDSS $r^{\prime} i^{\prime}$ filters were used on the Minicam and Keplercam. The "Harris" set of BVRI filters and a $U$ filter with a $\mathrm{CuSO}_{4}$ cell for red blocking were used for all CfA3 4Shooter observations. The same $U B V$ filters, and SDSS $r^{\prime} i^{\prime}$ filters were used on the Minicam and Keplercam. The $U$ filter broke in 2007 January and was replaced in 2007 June. A liquid leak was discovered in the $\mathrm{CuSO}_{4}$ cell of the $U$ filter in 2007 November and after repair and testing it was installed in 2008 February. These problems with the $U$ filter account for missing $U$-band photometry in 2007-2008.

The 64 4Shooter objects are all observed with the same camera and filters and reduced with the same pipeline, constituting one homogeneously observed and reduced sample. The 116 Keplercam objects also represent a homogeneously observed and reduced sample. The use of three different cameras and changing from $R I$ filters to $r^{\prime} i^{\prime}$ limits us from calling the entire CfA3 sample homogeneously observed and reduced. However, its acquisition and reduction can be called quasi homogeneous, since the $U B V$ filters were used on all three cameras, the detector responses are similar, and the same reduction pipeline was used.

\subsection{Observations}

Nearby $\mathrm{SNe}$ are discovered by both amateur and professional astronomers. Many of the discoverers promptly report their findings to the SN community via email. The IAU's Central Bureau for Astronomical Telegrams, the IAU Circulars and The Astronomer's Telegram are commonly used to disseminate information. Usually the initial discovery does not include spectroscopic confirmation and typing. The CfA Supernova Group depends on these discoveries, north of declination $-20^{\circ}$, for the SN it studies. The CfA3 discovery data are displayed in Table 1. Roughly two thirds of the CfA3 sample were discovered by professional observers. Roughly one third was discovered by amateurs, demonstrating their valuable contribution to nearby SN science. In first place, KAIT/LOTOSS/ LOSS discovered $46 \%$ of the CfA3 sample. In second place, the Puckett Observatory Supernova Search ${ }^{32}$ discovered $18 \%$. Most of these search surveys had typical limiting magnitudes of 19.5 mag. SDSS-II is the most obvious exception.

The CfA Supernova Group rapidly responds to new objects, acquiring spectra and optical and $J H K$ light curves. This allows for a deeper investigation into individual SN. For the CfA3 sample, we would sometimes initiate photometric observations

\footnotetext{
32 http://www.cometwatch.com/search.html
}

of untyped SN candidates, depending on their brightness and any additional properties provided in the email circulars, such as color or when the last nondetection of the $\mathrm{SN}$ candidate was made. If the SN candidate was brighter than 18-18.5 mag and north of $-20^{\circ}$ then we would take spectra with the FAST spectrograph. Our efforts have contributed roughly $40 \%$ of the reported identifications of SN type over the last six years. We did not follow any $\mathrm{SN}$ that had peak magnitudes fainter than $\sim 18.5 \mathrm{mag}$, making this the effective limiting magnitude for the CfA3 sample. However, this does not mean we observed every SN brighter than $\sim 18.5 \mathrm{mag}$.

With the information on type, age and any peculiar features in hand, either from our own spectra or from others' reports, a decision on whether to begin or continue observing the SN candidate was made. As one of our goals was to fill out the sampling across the whole range of SNe Ia (to provide a more complete training set for light-curve fitters), highest priority was given to SNe Ia that were young, slow-declining, fastdeclining, or otherwise peculiar. Another reason to prioritize slow decliners is that these are preferentially found at high redshift. Our program also observes core collapse SNe and high priority was given to stripped-envelope $\mathrm{SNe} \mathrm{IIb} / \mathrm{Ib} / \mathrm{c}$. Lower priority was given to SNe II and older SNe Ia. If a SN Ia was found to be older than $\sim 14$ days after $B$-band maximum at the time of our first observation then it was usually removed from our list.

We emphasize that the CfA3 sample distribution is not representative of the abundances of SN Ia type or host galaxies. Objects announced during the bright phase of the moon were also less likely to be included since spectroscopic identification was less likely to be obtained. The Keplercam and Minicam were usually mounted on the telescope at all phases of the moon while the 4Shooter was often taken off for several days around full moon. Our preference for young and more extreme events makes the CfA3 sample distribution less representative of the underlying population but does ensure that the wide range is being amply sampled. Finally, the limiting magnitude of both the searches and our follow up mean that highly reddened or intrinsically less-luminous SNe Ia are only observed in a small volume: they are severely under-represented in this sample compared to the cosmic rate.

In Figure 1, we plot redshift histograms of the CfA3 and OLD samples. The OLD sample is the nearby SN Ia sample as compiled in Jha et al. (2007). The CfA3 sample is primarily in the $0.02<z_{\mathrm{CMB}}<0.04$ region, where $z_{\mathrm{CMB}}$ is the redshift in the cosmic microwave background $(\mathrm{CMB})$ reference frame. The OLD sample is primarily below $z_{\mathrm{CMB}} \approx 0.03$. Above $z_{\mathrm{CMB}}=$ 0.01 , the median CfA3 and OLD redshifts are, respectively, 0.027 and 0.025 . Figure 2 shows the time of first observation, relative to $B$-band maximum light, with median values of -0.8 and -1.5 for CfA3 and OLD, respectively. The OLD sample has a higher percentage with very early observations. Respectively, the CfA3 and OLD samples have 48 and 47 objects with the time of first observation beginning sooner than 5 days before maximum, and 90 and 76 objects beginning before maximum.

The MLCS2k2 (Jha et al. 2007) light-curve parameter, $\Delta$, is roughly a measure of the relative $V$-band brightness compared to the $\Delta=0$ model light curve. Negative $\Delta$ means greater intrinsic luminosity and broader light curves and positive $\Delta$ means fainter luminosity and narrower light curves. Figure 3 shows the CfA3 distribution of $\Delta$ versus redshift above $z_{\mathrm{CMB}}=0.01$. The whole range of $\Delta$ is present out to $z_{\mathrm{CMB}} \approx 0.03$ and then the magnitude limits of discovery, spectroscopic identification, and 
Table 1

SN Ia Discovery Data

\begin{tabular}{|c|c|c|c|c|c|}
\hline SN Ia & Galaxy & Discovery Date (yyyy mm dd) & Position & CBET/IAUC & Discoverer \\
\hline $2001 \mathrm{C}$ & Anon Gal & 20010104 & $06: 59: 36.138+59: 31: 01.21$ & IAUC 7555 & Puckett, Sehgal \\
\hline $2001 \mathrm{G}$ & MCG +08-17-43 & 20010108 & 09:09:33.215 +50:16:50.83 & IAUC 7560 & Armstrong \\
\hline $2001 N$ & NGC 3327 & 20010121 & $10: 39: 58.060+24: 05: 25.68$ & IAUC 7568 & Chornock \\
\hline $2001 \mathrm{~V}$ & NGC 3987 & 20010219 & $11: 57: 24.910+25: 12: 09.49$ & IAUC 7585 & Berlind \\
\hline $2001 \mathrm{ah}$ & UGC 6211 & 20010327 & $11: 10: 29.838+55: 09: 39.03$ & IAUC 7603 & Puckett, Peoples \\
\hline 2001ay & IC 4423 & 20010418 & $14: 26: 16.943+26: 14: 55.24$ & IAUC 7611 & LOTOSS \\
\hline $2001 \mathrm{az}$ & UGC 10483 & 20010427 & $16: 34: 27.476+76: 01: 46.34$ & IAUC 7614 & Puckett, Peoples \\
\hline $2001 \mathrm{bf}$ & $\mathrm{MCG}+04-42-22$ & 20010503 & $18: 01: 34.059+26: 15: 01.82$ & IAUC 7620 & Armstrong \\
\hline $2001 \mathrm{cp}$ & UGC 10738 & 20010619 & $17: 11: 02.600+05: 50: 27.04$ & IAUC 7645 & LOTOSS \\
\hline 2001da & NGC 7780 & 20010709 & $23: 53: 32.741+08: 07: 02.20$ & IAUC 7658 & LOTOSS \\
\hline 2001eh & UGC 1162 & 20010909 & $01: 38: 12.056+41: 39: 18.95$ & IAUC 7712 & Armstrong \\
\hline 2001en & NGC 523 & 20010926 & $01: 25: 22.856+34: 01: 30.06$ & IAUC 7724 & LOTOSS; BAO \\
\hline 2001ep & NGC 1699 & 20011003 & 04:57:00.349-04:45:40.04 & IAUC 7727 & LOTOSS \\
\hline $2001 \mathrm{fe}$ & UGC 5129 & 20011102 & $09: 37: 57.021+25: 29: 40.84$ & IAUC 7742 & Armstrong \\
\hline $2001 \mathrm{fh}$ & Anon Gal & 20011103 & $21: 20: 42.538+44: 23: 53.14$ & IAUC 7744 & LOTOSS \\
\hline $2001 \mathrm{gb}$ & IC 582 & 20011120 & 09:59:00.960 +17:49:12.32 & IAUC 7758 & LOTOSS \\
\hline $2001 \mathrm{gc}$ & UGC 3375 & 20011121 & $05: 55: 26.111+51: 54: 34.22$ & IAUC 7759 & LOTOSS \\
\hline $2001 \mathrm{ic}$ & NGC 7503 & 20011207 & $23: 10: 43.298+07: 34: 10.25$ & IAUC 7770 & LOTOSS \\
\hline $2001 \mathrm{ie}$ & UGC 5542 & 20011209 & $10: 16: 50.954+60: 16: 53.32$ & IAUC 7771 & Bincoletto \\
\hline $2002 \mathrm{G}$ & Anon Gal & 20020118 & $13: 07: 55.285+34: 05: 07.09$ & IAUC 7797 & LOTOSS \\
\hline $2002 \mathrm{ar}$ & NGC 3746 & 20020203 & $11: 37: 43.863+22: 00: 34.47$ & IAUC 7819 & LOTOSS \\
\hline $2002 b f$ & Anon Gal & 20020222 & $10: 15: 42.314+55: 40: 07.35$ & IAUC 7836 & LOTOSS \\
\hline 2002 bo & NGC 3190 & 20020309 & $10: 18: 06.515+21: 49: 41.63$ & IAUC 7847 & Cacella; Hirose \\
\hline $2002 b z$ & $\mathrm{MCG}+05-34-33$ & 20020403 & $14: 24: 40.524+26: 37: 35.29$ & IAUC 7866 & Puckett, Gauthier \\
\hline $2002 \mathrm{~cd}$ & NGC 6916 & 20020408 & $20: 23: 34.402+58: 20: 47.30$ & IAUC 7871 & Armstrong \\
\hline $2002 \mathrm{ck}$ & UGC 10030 & 20020423 & $15: 47: 00.762-00: 59: 24.92$ & IAUC 7884 & LOTOSS \\
\hline $2002 \mathrm{cr}$ & NGC 5468 & 20020501 & $14: 06: 37.652-05: 26: 21.34$ & IAUC 7890 & Kushida \\
\hline 2002de & NGC 6104 & 20020601 & $16: 16: 30.334+35: 42: 30.09$ & IAUC 7914 & LOTOSS \\
\hline $2002 \mathrm{dj}$ & NGC 5018 & 20020612 & $13: 13: 00.414-19: 31: 08.56$ & IAUC 7918 & LOTOSS \\
\hline 2002do & MCG +07-41-1 & 20020617 & $19: 56: 12.853+40: 26: 10.47$ & IAUC 7923 & LOTOSS \\
\hline $2002 \mathrm{dp}$ & NGC 7678 & 20020618 & $23: 28: 30.103+22: 25: 38.05$ & IAUC 7924 & Klotz \\
\hline 2002es & UGC 2708 & 20020823 & $03: 23: 47.196+40: 33: 53.56$ & IAUC 7959 & LOTOSS \\
\hline $2002 \mathrm{eu}$ & Anon Gal & 20020830 & $01: 49: 43.549+32: 37: 42.31$ & IAUC 7963 & LOTOSS \\
\hline $2002 \mathrm{fb}$ & NGC 759 & 20020906 & $01: 57: 48.869+36: 20: 26.00$ & IAUC 7967 & LOTOSS \\
\hline $2002 \mathrm{fk}$ & NGC 1309 & 20020917 & $03: 22: 05.706-15: 24: 02.99$ & IAUC 7973 & Kushida; BAO \\
\hline 2002ha & NGC 6962 & 20021021 & $20: 47: 18.592+00: 18: 45.36$ & IAUC 7997 & LOTOSS \\
\hline 2002hd & $\mathrm{MCG}-01-23-8$ & 20021024 & 08:54:03.366 -07:11:21.48 & IAUC 7999 & LOTOSS \\
\hline 2002he & UGC 4322 & 20021028 & $08: 19: 58.804+62: 49: 13.71$ & IAUC 8002 & LOTOSS \\
\hline 2002hu & MCG +06-6-12 & 20021107 & $02: 18: 20.027+37: 27: 58.58$ & IAUC 8012 & Boles \\
\hline $2002 \mathrm{hw}$ & UGC 52 & 20021109 & $00: 06: 49.025+08: 37: 48.64$ & IAUC 8014 & LOTOSS \\
\hline 2002jy & NGC 477 & 20021217 & $01: 21: 16.231+40: 29: 55.27$ & IAUC 8035 & Arbour; Vanmunster \\
\hline $2002 \mathrm{kf}$ & Anon Gal & 20021227 & $06: 37: 15.283+49: 51: 10.87$ & IAUC 8040 & Brady \\
\hline $2003 \mathrm{D}$ & MCG -01-25-9 & 20030106 & 09:38:53.551-04:51:05.61 & IAUC 8043 & Puckett, Langoussis \\
\hline $2003 \mathrm{~K}$ & IC 1129 & 20030111 & $15: 32: 01.832+68: 14: 36.12$ & IAUC 8048 & Puckett, Langoussis \\
\hline $2003 \mathrm{U}$ & NGC 6365 & 20030127 & $17: 22: 45.626+62: 09: 50.67$ & IAUC 8059 & Boles \\
\hline $2003 \mathrm{~W}$ & UGC 5234 & 20030128 & $09: 46: 49.496+16: 02: 37.77$ & IAUC 8061 & LOTOSS \\
\hline $2003 \mathrm{ae}$ & Anon Gal & 20030123 & $09: 28: 22.570+27: 26: 41.29$ & IAUC 8066 & NEAT/Wood-Vasey et al. \\
\hline 2003ai & IC 4062 & 20030208 & $13: 00: 58.699+39: 51: 24.66$ & IAUC 8068 & LOTOSS \\
\hline $2003 \mathrm{cg}$ & NGC 3169 & 20030321 & $10: 14: 16.016+03: 28: 02.01$ & IAUC 8097 & Itagaki; Arbour \\
\hline $2003 \mathrm{ch}$ & UGC 3787 & 20030321 & $07: 17: 57.890+09: 41: 34.84$ & IAUC 8097 & LOTOSS \\
\hline $2003 \mathrm{cq}$ & NGC 3978 & 20030330 & $11: 56: 14.156+60: 31: 19.67$ & IAUC 8103 & Arbour \\
\hline 2003du & UGC 9391 & 20030422 & $14: 34: 35.728+59: 20: 03.93$ & IAUC 8121 & LOTOSS \\
\hline $2003 \mathrm{fa}$ & Anon Gal & 20030601 & $17: 44: 07.733+40: 52: 51.08$ & IAUC 8140 & LOTOSS \\
\hline 2003hu & Anon Gal & 20030906 & $19: 11: 31.121+77: 53: 34.91$ & IAUC 8196 & Armstrong \\
\hline $2003 i c$ & MCG -02-2-86 & 20030916 & $00: 41: 50.334-09: 18: 19.11$ & IAUC 8201 & LOTOSS \\
\hline 2003 it & UGC 40 & 20031013 & $00: 05: 48.523+27: 27: 08.62$ & IAUC 8225 & Puckett, Cox \\
\hline 2003iv & $\mathrm{MCG}+02-8-14$ & 20031017 & $02: 50: 07.244+12: 50: 45.29$ & IAUC 8226 & LOTOSS \\
\hline $2003 \mathrm{kc}$ & MCG +05-23-37 & 20031121 & $09: 46: 34.293+30: 39: 19.27$ & IAUC 8242 & LOSS \\
\hline $2003 \mathrm{kf}$ & MCG -02-16-2 & 20031127 & $06: 04: 35.484-12: 37: 42.87$ & IAUC 8245 & LOSS \\
\hline $2004 \mathrm{~K}$ & ESO 579-G22 & 20040119 & $14: 23: 39.802-19: 26: 50.13$ & IAUC 8273 & LOSS \\
\hline $2004 \mathrm{~L}$ & $\mathrm{MCG}+03-27-38$ & 20040121 & $10: 27: 04.125+16: 01: 07.80$ & IAUC 8274 & LOSS \\
\hline 2004ap & Anon Gal & 20040308 & $10: 05: 43.813+10: 16: 16.68$ & IAUC 8300 & LOSS \\
\hline 2004as & Anon Gal & 20040311 & $11: 25: 39.185+22: 49: 49.05$ & IAUC 8302 & LOSS \\
\hline $2004 b g$ & UGC 6363 & 20040407 & $11: 21: 01.542+21: 20: 22.95$ & IAUC 8317 & Armstrong \\
\hline $2004 \mathrm{ef}$ & UGC 12158 & 20040904 & $22: 42: 10.021+19: 59: 39.89$ & IAUC 8399 & Boles; Armstrong \\
\hline
\end{tabular}


Table 1

(Continued)

\begin{tabular}{|c|c|c|c|c|c|}
\hline SN Ia & Galaxy & Discovery Date (yyyy mm dd) & Position & CBET/IAUC & Discoverer \\
\hline $2004 f u$ & NGC 6949 & 20041104 & $20: 35: 11.608+64: 48: 26.41$ & IAUC 8428 & Arbour \\
\hline $2005 \mathrm{M}$ & NGC 2930 & 20050119 & $09: 37: 32.356+23: 12: 02.20$ & IAUC 8470 & Puckett, George \\
\hline $2005 \mathrm{am}$ & NGC 2811 & 20050222 & 09:16:13.087 -16:18:15.97 & IAUC 8490 & Martin \\
\hline $2005 \mathrm{cf}$ & MCG -01-39-3 & 20050528 & $15: 21: 32.225-07: 24: 47.66$ & CBET 158 & LOSS \\
\hline $2005 \mathrm{dv}$ & NGC 5283 & 20050904 & $13: 41: 04.478+67: 40: 19.53$ & CBET 217 & Dainese, Dimai \\
\hline $2005 \mathrm{el}$ & NGC 1819 & 20050925 & 05:11:48.744 +05:11:39.19 & CBET 233 & LOSS \\
\hline 2005 eq & MCG -01-9-6 & 20050930 & 03:08:49.357 -07:02:00.24 & IAUC 8608 & LOSS \\
\hline $2005 \mathrm{eu}$ & Anon Gal & 20051004 & $02: 27: 43.239+28: 10: 36.71$ & CBET 242 & LOSS \\
\hline 2005 ew & Anon Gal & 20051004 & $03: 39: 23.747+35: 02: 49.38$ & CBET 244 & Nearby SN Factory \\
\hline $2005 \mathrm{hc}$ & MCG +00-6-3 & 20051012 & $01: 56: 47.950-00: 12: 49.42$ & CBET 259 & SDSS-II \\
\hline $2005 \mathrm{hf}$ & Anon Gal & 20051025 & $01: 27: 05.991+19: 07: 00.83$ & IAUC 8622 & Quimby et al. \\
\hline 2005hj & Anon Gal & 20051026 & 01:26:48.397-01:14:17.30 & CBET 266 & Quimby et al. \\
\hline $2005 \mathrm{hk}$ & UGC 272 & 20051030 & $00: 27: 50.879-01: 11: 53.32$ & IAUC 8625 & SDSS-II; LOSS \\
\hline 2005 iq & $\mathrm{MCG}-03-1-8$ & 20051105 & $23: 58: 32.422-18: 42: 32.97$ & IAUC 8628 & LOSS \\
\hline $2005 \mathrm{ir}$ & Anon Gal & 20051028 & $01: 16: 43.796+00: 47: 40.89$ & CBET 277 & SDSS II; Quimby et al. \\
\hline $2005 \mathrm{kc}$ & NGC 7311 & 20051109 & $22: 34: 07.308+05: 34: 06.04$ & IAUC 8629 & Puckett, Sostero \\
\hline $2005 \mathrm{ke}$ & NGC 1371 & 20051113 & 03:35:04.356-24:56:38.93 & IAUC 8630 & LOSS \\
\hline $2005 \mathrm{ki}$ & NGC 3332 & 20051118 & $10: 40: 28.219+09: 12: 08.21$ & IAUC 8632 & LOSS \\
\hline $20051 \mathrm{~s}$ & MCG +07-7-1 & 20051209 & $02: 54: 15.914+42: 43: 29.15$ & IAUC 8643 & Armstrong \\
\hline 2005lu & MCG -03-07-40 & 20051211 & 02:36:03.753-17:15:49.50 & IAUC 8645 & LOSS \\
\hline $20051 \mathrm{z}$ & UGC 1666 & 20051224 & $02: 10: 49.727+34: 58: 57.84$ & CBET 329 & Puckett, Gagliano \\
\hline $2005 \mathrm{mc}$ & UGC 4414 & 20051223 & $08: 27: 06.277+21: 38: 46.61$ & CBET 331 & THCA Supernova Survey \\
\hline $2005 \mathrm{~ms}$ & UGC 4614 & 20051227 & $08: 49: 14.320+36: 07: 46.72$ & CBET 343 & Puckett, Kroes \\
\hline $2005 \mathrm{mz}$ & NGC 1275 & 20051231 & $03: 19: 49.910+41: 30: 18.86$ & CBET 347 & Newton, Peoples, Puckett \\
\hline $2005 \mathrm{na}$ & UGC 3634 & 20051231 & $07: 01: 36.659+14: 07: 58.75$ & CBET 350 & Newton, Ceravolo, Pucket \\
\hline 2006B & UGC 12538 & 20060108 & $23: 21: 09.803+33: 24: 00.74$ & CBET 356 & Puckett, Sostero \\
\hline $2006 \mathrm{D}$ & MCG -01-33-34 & 20060111 & 12:52:33.871-09:46:30.56 & CBET 362 & Colesanti, et al. \\
\hline $2006 \mathrm{H}$ & Anon Gal & 20060115 & $03: 26: 01.533+40: 41: 41.69$ & CBET 367 & Puckett, Sostero \\
\hline $2006 N$ & $\mathrm{MCG}+11-8-12$ & 20060121 & $06: 08: 31.268+64: 43: 24.82$ & CBET 375 & Armstrong \\
\hline $2006 \mathrm{~S}$ & UGC 7934 & 20060126 & $12: 45: 39.033+35: 05: 12.16$ & CBET 379 & Puckett, Gagliano \\
\hline $2006 X$ & NGC 4321 & 20060204 & $12: 22: 53.911+15: 48: 31.65$ & IAUC 8667 & Suzuki; Migliardi \\
\hline $2006 \mathrm{ac}$ & NGC 4619 & 20060209 & $12: 41: 44.894+35: 04: 07.93$ & IAUC 8669 & LOSS \\
\hline 2006ah & Anon Gal & 20060209 & $13: 46: 13.540-09: 07: 51.92$ & CBET 402 & Nearby SN Factory \\
\hline 2006ak & Anon Gal & 20060217 & $11: 09: 32.640+28: 37: 51.63$ & CBET 408 & Tyurina, Lipunov et al. \\
\hline 2006al & Anon Gal & 20060219 & $10: 39: 28.238+05: 11: 00.39$ & IAUC 8677 & Holmes, Devore \\
\hline 2006an & Anon Gal & 20060221 & $12: 14: 38.749+12: 13: 47.75$ & CBET 413 & Quimby, Castro \\
\hline 2006ar & $\mathrm{MCG}+11-13-36$ & 20060305 & $10: 37: 30.616+65: 00: 57.78$ & CBET 420 & Boles \\
\hline 2006ax & NGC 3663 & 20060320 & $11: 24: 03.432-12: 17: 29.52$ & CBET 435 & LOSS \\
\hline 2006az & NGC 4172 & 20060323 & $12: 12: 14.650+56: 10: 47.11$ & IAUC 8691 & Newton, Cox, Puckett \\
\hline $2006 \mathrm{bb}$ & UGC 4468 & 20060325 & $08: 33: 31.096+41: 31: 04.20$ & CBET 444 & Puckett, Gagliano \\
\hline 2006bd & UGC 6609 & 20060326 & $11: 38: 28.420+20: 31: 34.45$ & CBET 448 & Puckett, Cox \\
\hline 2006bk & $\mathrm{MCG}+06-23-20$ & 20060403 & $15: 04: 33.606+35: 57: 50.53$ & CBET 462 & Boles \\
\hline $2006 \mathrm{bq}$ & NGC 6685 & 20060423 & $18: 39: 58.941+39: 58: 56.34$ & CBET 479 & Puckett, Pelloni \\
\hline $2006 \mathrm{br}$ & NGC 5185 & 20060425 & $13: 30: 01.716+13: 24: 56.61$ & CBET 482 & Puckett, Sostero \\
\hline $2006 \mathrm{bt}$ & Anon Gal & 20060426 & $15: 56: 30.526+20: 02: 45.34$ & CBET 485 & LOSS \\
\hline 2006bu & Anon Gal & 20060427 & $13: 52: 47.736+05: 18: 48.41$ & CBET 490 & Holmes, Devore, Graves \\
\hline 2006bw & Anon Gal & 20060427 & $14: 33: 56.806+03: 47: 55.82$ & CBET 497 & LOSS \\
\hline $2006 \mathrm{bz}$ & Anon Gal & 20060504 & $13: 00: 43.362+27: 57: 41.28$ & IAUC 8707 & LOSS \\
\hline $2006 \mathrm{cc}$ & UGC 10244 & 20060506 & $16: 09: 56.460+43: 07: 35.89$ & CBET 505 & LOSS \\
\hline $2006 \mathrm{cf}$ & UGC 6015 & 20060511 & $10: 54: 02.585+46: 01: 36.44$ & IAUC 8710 & LOSS; Puckett, Toth \\
\hline $2006 \mathrm{cg}$ & Anon Gal & 20060506 & $13: 05: 02.382+28: 44: 25.11$ & CBET 509 & Quimby, Mondol \\
\hline $2006 \mathrm{cj}$ & Anon Gal & 20060517 & $12: 59: 24.519+28: 20: 51.36$ & CBET 515 & Quimby, Mondol, Castro \\
\hline $2006 \mathrm{~cm}$ & UGC 11723 & 20060524 & 21:20:17.423-01:41:02.08 & CBET 521 & Puckett, Langoussis \\
\hline $2006 \mathrm{cp}$ & UGC 7357 & 20060528 & $12: 19: 14.890+22: 25: 37.89$ & CBET 524 & LOSS \\
\hline $2006 \mathrm{cq}$ & IC 4239 & 20060529 & $13: 24: 25.040+30: 57: 22.32$ & CBET 527 & Newton, Briggs, Puckett \\
\hline $2006 \mathrm{cs}$ & $\mathrm{MCG}+06-30-79$ & 20060603 & $13: 45: 33.879+35: 36: 36.58$ & CBET 536 & LOSS \\
\hline $2006 \mathrm{cz}$ & $\mathrm{MCG}-01-38-2$ & 20060614 & $14: 48: 36.643-04: 44: 30.91$ & IAUC 8721 & LOSS \\
\hline $2006 \mathrm{ef}$ & NGC 809 & 20060818 & $02: 04: 19.529-08: 43: 42.50$ & CBET 597 & LOSS \\
\hline $2006 \mathrm{ej}$ & NGC 191 & 20060823 & 00:38:59.812-09:00:57.43 & CBET 603 & LOSS \\
\hline $2006 \mathrm{em}$ & NGC 911 & 20060825 & $02: 25: 44.313+41: 56: 31.55$ & CBET 605 & LOSS \\
\hline 2006en & MCG +05-54-41 & 20060826 & $23: 10: 05.053+30: 13: 23.82$ & CBET 606 & Puckett, Peoples \\
\hline $2006 \mathrm{et}$ & NGC 232 & 20060903 & $00: 42: 45.779-23: 33: 29.80$ & CBET 616 & Itagaki \\
\hline 2006eu & $\mathrm{MCG}+08-36-16$ & 20060903 & $20: 02: 51.147+49: 19: 02.18$ & CBET 618 & LOSS \\
\hline $2006 \mathrm{ev}$ & UGC 11758 & 20060912 & $21: 30: 59.329+13: 59: 21.30$ & IAUC 8747 & Ory \\
\hline
\end{tabular}


Table 1

(Continued)

\begin{tabular}{|c|c|c|c|c|c|}
\hline SN Ia & Galaxy & Discovery Date (yyyy mm dd) & Position & CBET/IAUC & Discoverer \\
\hline $2006 \mathrm{gj}$ & UGC 2650 & 20060918 & $03: 17: 35.718-01: 41: 30.18$ & CBET 631 & Puckett, Toth \\
\hline $2006 \mathrm{gr}$ & UGC 12071 & 20060821 & $22: 32: 22.677+30: 49: 43.80$ & CBET 638 & LOSS \\
\hline $2006 \mathrm{gt}$ & Anon Gal & 20060918 & $00: 56: 17.318-01: 37: 46.66$ & CBET 641 & Quimby, Mondol \\
\hline 2006ha & IC 1461 & 20060927 & $22: 58: 34.280+15: 10: 25.53$ & CBET 649 & LOSS \\
\hline $2006 \mathrm{hb}$ & MCG -04-12-34 & 20060927 & 05:02:01.302-21:07:55.18 & CBET 649 & LOSS \\
\hline $2006 \mathrm{hn}$ & UGC 6154 & 20060928 & $11: 07: 18.439+76: 41: 50.52$ & CBET 653 & Sehgal, Gagliano, Puckett \\
\hline 2006is & Anon Gal & 20060918 & $05: 17: 34.372-23: 46: 54.67$ & CBET 659 & LOSS \\
\hline $2006 \mathrm{je}$ & IC 1735 & 20061015 & $01: 50: 53.264+33: 05: 53.27$ & CBET 675 & LOSS \\
\hline $2006 \mathrm{ke}$ & UGC 3365 & 20061019 & $05: 52: 37.391+66: 49: 00.78$ & CBET 682 & LOSS \\
\hline $2006 \mathrm{kf}$ & UGC 2829 & 20061021 & $03: 41: 50.472+08: 09: 24.93$ & CBET 686 & LOSS \\
\hline 2006le & UGC 3218 & 20061026 & $05: 00: 41.934+62: 15: 18.98$ & CBET 700 & LOSS \\
\hline $20061 f$ & UGC 3108 & 20061026 & $04: 38: 29.511+44: 02: 01.82$ & CBET 704 & LOSS \\
\hline $2006 \mathrm{mo}$ & MCG +06-02-17 & 20061101 & $00: 46: 38.479+36: 19: 57.70$ & CBET 719 & LOSS \\
\hline $2006 \mathrm{mp}$ & MCG +08-31-29 & 20061103 & $17: 12: 00.194+46: 33: 21.54$ & CBET 720 & Puckett, Gagliano \\
\hline $2006 \mathrm{mq}$ & ESO 494-G26 & 20061022 & 08:06:12.378 -27:33:45.38 & CBET 721 & LOSS \\
\hline $2006 \mathrm{nz}$ & Anon Gal & 20061108 & $00: 56: 29.206-01: 13: 35.90$ & CBET 743 & SDSS-II \\
\hline $20060 \mathrm{a}$ & Anon Gal & 20061111 & $21: 23: 42.939-00: 50: 36.50$ & CBET 743 & SDSS-II \\
\hline $2006 \mathrm{ob}$ & Anon Gal & 20061113 & $01: 51: 48.133+00: 15: 48.46$ & CBET 743 & SDSS-II \\
\hline 2006on & Anon Gal & 20061111 & $21: 55: 58.482-01: 04: 12.79$ & CBET 745 & SDSS-II \\
\hline 2006or & NGC 3891 & 20061118 & $11: 48: 03.469+30: 21: 23.02$ & CBET 749 & Puckett, Kroes \\
\hline $2006 \mathrm{os}$ & UGC 2384 & 20061121 & $02: 55: 00.998+16: 00: 35.26$ & CBET 751 & Quimby, Castro \\
\hline 2006 ot & ESO 544-G31 & 20061122 & $02: 15: 04.800-20: 45: 58.97$ & CBET 754 & LOSS (Joubert, Li) \\
\hline 2006qo & UGC 4133 & 20061129 & 08:00:08.422 +56:22:07.25 & CBET 763 & Joubert, Li (LOSS) \\
\hline $2006 s r$ & UGC 14 & 20061212 & $00: 03: 35.024+23: 11: 45.67$ & IAUC 8784 & Rich \\
\hline $2006 t d$ & Anon Gal & 20061224 & $01: 58: 15.761+36: 20: 57.76$ & CBET 787 & Kloehr \\
\hline 2006te & Anon Gal & 20061228 & $08: 11: 42.963+41: 33: 16.80$ & CBET 791 & Trondal, Luckas, Schwartz \\
\hline $2007 \mathrm{~F}$ & UGC 8162 & 20070111 & $13: 03: 15.059+50: 37: 07.53$ & CBET 803 & Puckett, Gagliano \\
\hline $2007 \mathrm{H}$ & Anon Gal & 20070110 & 08:35:02.009-08:20:16.00 & CBET 806 & Joubert, Li (LOSS) \\
\hline $2007 \mathrm{~N}$ & MCG -01-33-12 & 20070121 & $12: 49: 01.212-09: 27: 10.77$ & CBET 818 & Lee, Li (LOSS) \\
\hline 20070 & UGC 9612 & 20070121 & $14: 56: 05.161+45: 24: 17.37$ & CBET 818 & Lee, Li (LOSS) \\
\hline $2007 \mathrm{R}$ & UGC 4008 & 20070126 & $07: 46: 37.513+44: 47: 22.51$ & CBET 823 & Puckett, Gray \\
\hline $2007 \mathrm{~S}$ & UGC 5378 & 20070129 & $10: 00: 31.237+04: 24: 25.26$ & CBET 825 & Puckett, Gorelli \\
\hline $2007 \mathrm{ae}$ & UGC 10704 & 20070219 & $17: 01: 52.067+79: 01: 54.26$ & CBET 856 & Nissinen, Hentunen \\
\hline 2007af & NGC 5584 & 20070301 & $14: 22: 21.064-00: 23: 37.92$ & CBET 863 & Itagaki \\
\hline 2007ai & MCG -04-38-4 & 20070306 & $16: 12: 53.740-21: 37: 48.57$ & CBET 870 & $\mathrm{Li}(\mathrm{LOSS})$ \\
\hline 2007al & Anon Gal & 20070310 & 09:59:18.467 -19:28:25.39 & CBET 875 & Madison, Li (LOSS) \\
\hline 2007ap & MCG +03-41-3 & 20070313 & $15: 56: 23.035+16: 30: 57.92$ & CBET 883 & Puckett, Kroes \\
\hline 2007ar & MCG +10-19-62 & 20070312 & $13: 21: 01.797+58: 33: 02.80$ & CBET 886 & Duszanowicz \\
\hline $2007 \mathrm{au}$ & UGC 3725 & 20070318 & $07: 11: 46.095+49: 51: 13.08$ & CBET 895 & Lee, Li (LOSS) \\
\hline $2007 a x$ & NGC 2577 & 20070321 & $08: 22: 43.242+22: 33: 16.91$ & CBET 904 & Arbour \\
\hline $2007 \mathrm{ba}$ & UGC 9798 & 20070329 & $15: 16: 42.581+07: 23: 47.91$ & CBET 911 & Winslow, Li (LOSS) \\
\hline $2007 \mathrm{bc}$ & UGC 6332 & 20070404 & $11: 19: 14.566+20: 48: 32.26$ & CBET 913 & Prasad, Li (LOSS) \\
\hline 2007bd & UGC 4455 & 20070404 & $08: 31: 33.375-01: 11: 57.73$ & CBET 914 & Prasad, Li (LOSS) \\
\hline $2007 \mathrm{bm}$ & NGC 3672 & 20070420 & 11:25:02.309-09:47:53.96 & CBET 936 & Martin \\
\hline $2007 \mathrm{bz}$ & IC 3918 & 20070422 & $12: 56: 53.764+22: 22: 23.12$ & CBET 941 & Nearby SN Factory \\
\hline $2007 \mathrm{ca}$ & MCG -02-34-61 & 20070425 & $13: 31: 05.840-15: 06: 06.52$ & CBET 945 & Prasad, Li \\
\hline $2007 \mathrm{cg}$ & ESO 508-G75 & 20070511 & $13: 25: 33.588-24: 39: 08.29$ & CBET 960 & Thrasher, Li (LOSS) \\
\hline $2007 \mathrm{ci}$ & NGC 3873 & 20070515 & $11: 45: 45.851+19: 46: 13.74$ & CBET 966 & Puckett, Crowley \\
\hline $2007 \mathrm{co}$ & $\mathrm{MCG}+05-43-16$ & 20070604 & $18: 23: 03.599+29: 53: 49.39$ & CBET 977 & Nicolas \\
\hline $2007 \mathrm{cp}$ & IC 807 & 20070613 & $12: 42: 12.748-17: 24: 07.45$ & CBET 980 & Khandrika, Li (LOSS) \\
\hline $2007 \mathrm{cq}$ & Anon Gal & 20070621 & $22: 14: 40.423+05: 04: 48.57$ & CBET 983 & Orff, Newton \\
\hline $2007 \mathrm{qe}$ & Anon Gal & 20071113 & $23: 54: 12.958+27: 24: 33.02$ & CBET 1138 & Yuan et al. (ROTSE) \\
\hline $2007 \mathrm{sr}$ & NGC 4038 & 20071218 & $12: 01: 52.800-18: 58: 21.83$ & CBET 1172 & Drake et al. \\
\hline $2008 \mathrm{~L}$ & NGC 1259 & 20080114 & $03: 17: 16.596+41: 22: 56.23$ & CBET 1212 & Fujita \\
\hline 2008af & UGC 9640 & 20080209 & $14: 59: 28.493+16: 39: 11.77$ & CBET 1248 & Boles \\
\hline $2008 \mathrm{bf}$ & NGC 4055 & 20080318 & $12: 04: 02.877+20: 14: 42.29$ & CBET 1307 & Parisky (LOSS) \\
\hline
\end{tabular}

Notes. Positions are a weighted mean of our measured SN positions, usually in $R / r^{\prime}$ but occasionally from $V$ when insufficient $R / r^{\prime}$ data were available. These are generally an improvement over the positions reported by the discoverer.

photometric-follow-up decisions discriminate against fainter objects which are not present in our sample at higher redshifts. The roughly diagonal slope in the right edge of the $\Delta$-versusredshift distribution is consistent with a limiting peak magnitude of $\sim 18.5$ mag in the CfA3 objects.
The FLWO $1.2 \mathrm{~m}$ telescope has its time allocated to a specific observing program each night with the requirement that roughly $10 \%$ of the night be devoted to other programs' observations. From 2001 to summer, 2005, our typical time allocation was one night per month with a few months of 


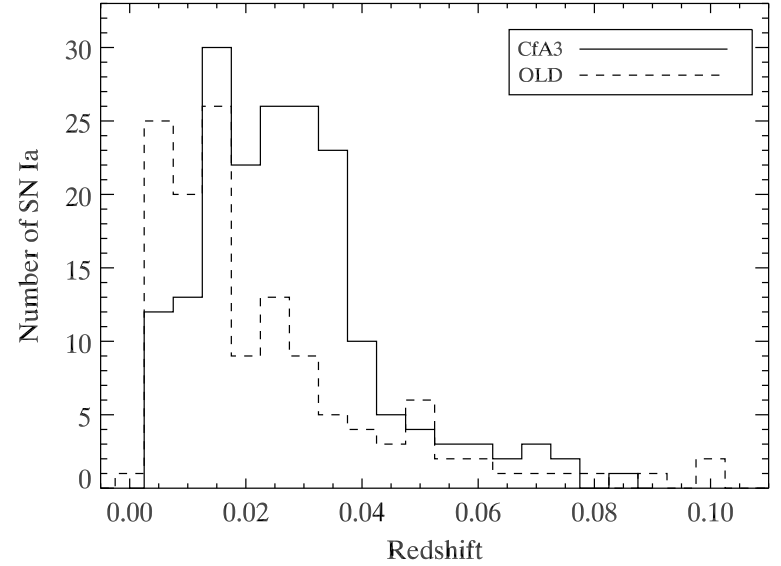

Figure 1. Histograms of redshift $\left(z_{\mathrm{CMB}}\right)$ for both CfA3 and OLD SNe Ia. The mean redshifts are, respectively, 0.027 and 0.024 . There is one OLD SN above $z=0.12$ not shown

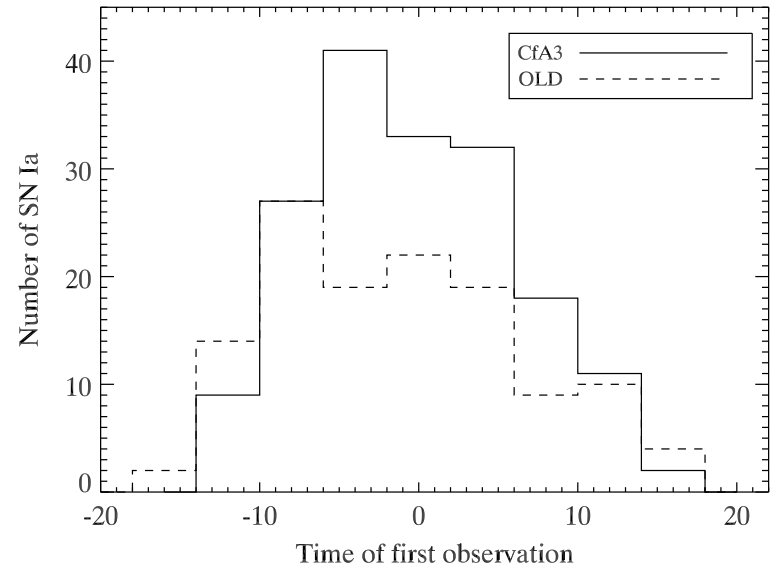

Figure 2. Histograms of time of first observation in the rest frame, relative to maximum light in $B$, as calculated by MLCS2k2. OLD has more objects with very early measurements which are useful for constraining the rise time and better understanding the explosion mechanism.

multiple nights to acquire calibration and host-galaxy reference images. Nightly requests of two $\mathrm{SNe}$ to other observers were typical during this period. Beginning in the fall of 2005, two changes significantly increased both the number of SNe we observed and the sampling per object. Instead of a single night per month, we received roughly seven nights per month. Additionally, several other observing programs made significant numbers of SN observations for us in time they could not use. The most notable group was the CfA component of the Kepler Mission. ${ }^{33}$ We tried to observe new, high-priority $\mathrm{SNe}$ every one or two nights until $\sim 10$ days past maximum light and less frequently thereafter. Weather and competing targets sometimes reduced the actual cadence. Secondary standards from Landolt (1992) and Smith et al. (2002) were observed on photometric nights and reference images for host-galaxy subtraction were obtained after the SN had faded sufficiently, usually a year after maximum light. Figure 4 shows a histogram of the number of nights observed for each $\mathrm{SN}$ in the CfA3 sample. The mean number is 15 and the median is 12 . The number of objects with 20-or-more nights of observation is 45 and the number with 10-or-more nights is 121 .

\footnotetext{
33 http://kepler.nasa.gov/
}

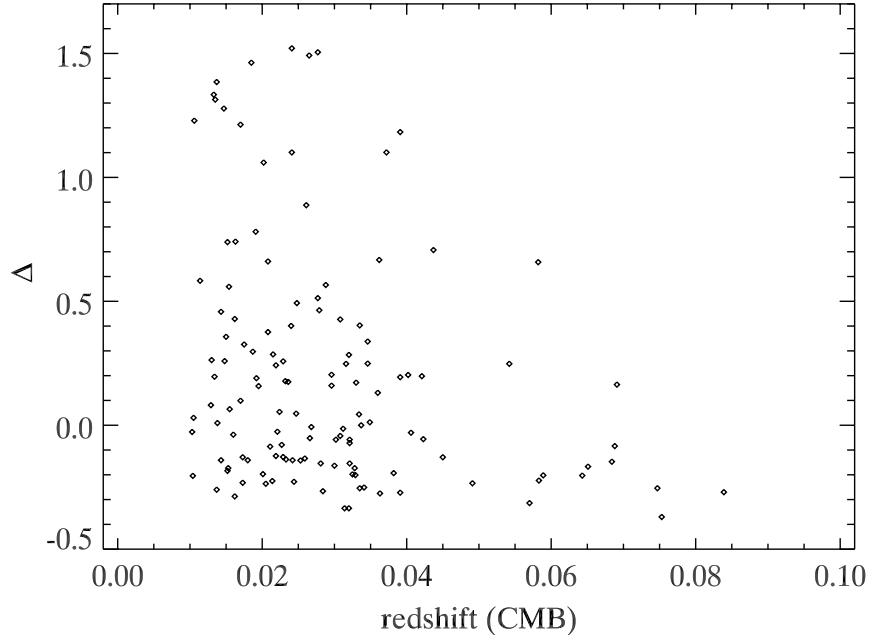

Figure 3. Plot of MLCS2k2 $\Delta$ vs. redshift (CMB) for the CfA3 sample. Objects closer than $z=0.01$ are not shown. The highest redshift for a given $\Delta$ is consistent with an approximate, effective peak limiting magnitude of $18.5 \mathrm{mag}$. At high redshift, in H09, no objects with $\Delta>0.75$ are found in the ESSENCE, SNLS, and Higher-Z samples used.

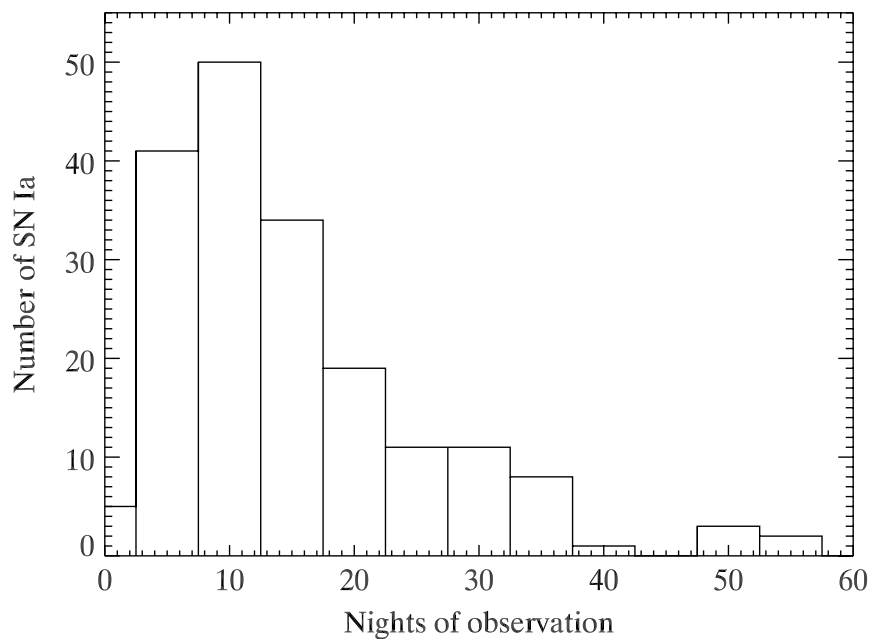

Figure 4. Histogram of the number of nights each CfA3 SN Ia was observed in $R / r^{\prime}$ band, representative of $V$ and $i^{\prime}$ also. $B$ is slightly less. $U$ is often much less as it fades first, or nonexistent for when the filter was broken. The mean is 15 nights and the median is 12 . There are 121 objects with 10 or more nights and 45 with 20 or more.

\subsection{Pipeline: Reduction Stage}

In this stage, raw images are processed to the point where all their star-like objects have had their flux measured, but not yet calibrated. Images first undergo bias subtraction and flat fielding. Dome-screen flats were used for $B V R I r^{\prime} i^{\prime}$ while twilight flats were used for $U$. The 4 Shooter images had their bad pixels masked out while the Minicam and Keplercam images did not require this. The small, but non-negligible, $I$-band fringes on the 4 Shooter were removed to the extent possible by subtracting fringe frames created from several nights of $I$-band images. The $i^{\prime}$-band fringes on the Minicam and Keplercam were much smaller in amplitude, making fringe correction unnecessary.

The cosmic-ray removal algorithm, la_cosmic (van Dokkum 2001), in the form of the IDL code, la_cosmic.pro, by Joshua Bloom, was then applied to the flat-fielded images to remove most of the cosmic rays. It uses a two-dimensional Laplacian algorithm to detect cosmic rays. Although removing the cosmic rays did not have a significant effect on the photometry and 
reference-image subtraction, this step was applied to each image.

A linear astrometric solution was calculated for each image. We used astrometric solutions based on an external astrometric catalog for a handful of good-seeing images of a single field. We then ran SWarp (Bertin et al. 2002) on these images to properly scale and align them, and center them on the $\mathrm{SN}$ position. DoPHOT (Schechter et al. 1993) was used to get the field star positions to make an "internal" astrometric catalog from our own images. We then reran these same images through these same stages with the internal astrometric catalog and recalculated the field star positions to make our final internal astrometric catalog. This was done for each SN field.

The UCAC2 catalog (Zacharias et al. 2004) was our preferred external catalog but it does not extend above declinations of roughly $+45^{\circ}$. Where the UCAC2 catalog was sparsely populated, we used either the USNO-B1.0 (Monet et al. 2003) or USNO-A2.0 catalogs (Monet et al. 1998). UCAC2 has an accuracy of around $0^{\prime \prime}$.03 while USNO-B1.0 and USNO-A2.0 have poorer accuracies of roughly $0^{\prime \prime} .20$ and $0^{\prime \prime} .25$, respectively. The resulting average standard deviation and relative accuracy of the star coordinates in our internal astrometric catalogs did not depend significantly on which external catalog was used. The absolute accuracy of our internal catalogs will be better in those that used UCAC2 for the initial solution but our positions will generally be better than those reported at discovery. Since we are primarily interested in relative accuracy, though, all our internal astrometric catalogs are adequate. The typical standard deviation of a star's position in our internal astrometric catalogs is 0.06 .

We then used our internal astrometric catalogs to create a linear astrometric solution for all of the flat-fielded images. A linear solution was adequate for the small field of view of the $1.2 \mathrm{~m}$ images. The astrometric solution was used in SWarp to align the images to a common pixel system so that host-galaxy reference images can be subtracted. DoPHOT was run on the SWarped images to calculate fluxes for all stellar-shaped objects.

DoPHOT uses a parameterized point-spread function (PSF) model. A range of functions can be effectively chosen by setting different values of the DoPHOT PSF-shape parameters $\alpha$ and $\beta$. With the PSF function set, DoPHOT first fits for a single PSF shape and size over the whole image. The high signal-to-noise ratio $(\mathrm{S} / \mathrm{N})$ stars most heavily influence the best-fit PSF in DoPHOT. Then it fits this PSF to each starlike detection, calculating a best-fit position, sky value and flux amplitude. It is important that the PSF model be capable of fitting the actual PSF shape of the data. We found that an order-2 Moffat fit our stars' PSF well while the default, truncated Gaussian underestimated the flux in the wings of the stars.

A mismatched PSF function will do a better job of fitting low-S/N stars than of fitting high-S/N stars (since low-S/N data is less constraining), possibly introducing relative inaccuracies between the faint and bright stars. We compared our DoPHOT truncated-Gaussian-PSF magnitudes with aperture-photometry magnitudes and found that the DoPHOT magnitudes differed from the aperture-photometry magnitudes by about $0.01-0.02$ mag per mag. The fainter stars were being interpreted as fainter relative to the aperture photometry magnitudes than were the bright stars. When we used the well matching, order-2 Moffat function for our PSF, this effect was drastically diminished.

In Section 2.5, we describe the calibration process to generate photometric catalogs for the comparison stars in the SN fields. To calculate a photometric zero point for each SN image, we took a weighted mean of the differences between our catalog magnitudes (in the natural system) and the DoPHOT measurements of the comparison stars. In the cases where the $\mathrm{SN}$ is sufficiently distant from any underlying structure (such as host-galaxy light or neighboring stars) the DoPHOT magnitudes of the SN can be combined with their respective image zero points to produce a calibrated light curve in the natural system.

\subsection{Pipeline: Host-Galaxy Subtraction}

Most of the $\mathrm{SNe}$ in our sample required host-galaxy subtraction. Reference images were acquired on clear nights with good seeing and little or no moon so as to maximize their $\mathrm{S} / \mathrm{N}$. We also took reference images of $\mathrm{SNe}$ that did not need host subtraction as a way to test the host-subtraction process.

Accurately subtracting the reference image from the SN image which was obtained under different seeing conditions requires a convolution kernel that can transform the PSF of one image to the PSF of the other. The convolution kernel is calculated using the algorithm of Alard \& Lupton (1998) and Alard (2000) with slight improvements as in Becker et al. (2004) and Miknaitis et al. (2007). The two images are each divided into stamps and substamps and the best-fit convolution kernel is determined. The image with a narrower PSF is convolved to the other image. Usually the reference image was convolved but sometimes the SN image was. The SN flux in the difference image is measured with the DoPHOT PSF from the stars of the (wider) unconvolved image.

All of the reference images for the Keplercam SN Ia were obtained with the Keplercam, resulting in "same-camera" subtractions. Some of the reference images for the 4Shooter and Minicam SN Ia were taken with the Keplercam, resulting in "cross-camera" subtractions. The responsivity of the different cameras are similar enough in a given passband so there is no problem in using the Keplercam reference images for 4Shooter and Minicam SN images. The flux normalization for the difference image can be chosen from either the $\mathrm{SN}$ image or the reference image. In the case of the same-camera subtractions, we chose to use the flux normalization from the reference image so that this would be used for every observation of that $\mathrm{SN}$ in a given band. In the cross-camera subtractions, the flux normalization from the SN image was used in order to stay in the natural system of the camera in which the SN data were observed. If the unconvolved image happens to be the one chosen for the flux normalization of the difference image then its zeropoint magnitude can be directly applied to the DoPHOT SN magnitude to achieve the calibrated natural-system SN magnitude. If the zero point of the image-that-got-convolved is used for the flux normalization then the flux of the SN in the difference image must be divided by the normalization (sum) of the convolution kernel to preserve the pre-convolution flux scale.

Noise maps are propagated for both images and are used to calculate a noise map for the difference image. Information from the noise image is combined with the DoPHOT uncertainty and calibration uncertainty to produce the uncertainty of the natural system SN measurement.

\subsection{Calibration}

On photometric nights, we observed one or two fields of secondary standards every hour, over a range in air mass 
that matched the $\mathrm{SN}$ observations. For the UBVRI bands used on the 4Shooter, we used secondary standards from Landolt (1992). Smith et al. (2002) establish the photometric system for the SDSS passbands, $u^{\prime} g^{\prime} r^{\prime} i^{\prime} z^{\prime}$. They use many of the fields from Landolt (1992) but much fewer stars. For the Minicam and Keplercam, where we are using $U B V r^{\prime} i^{\prime}$, we chose our secondary standards from Smith et al. (2002) to ensure that we have stars with $r^{\prime} i^{\prime}$ calibration and used the $U B V$ magnitudes from Landolt (1992).

We performed aperture photometry on the Landolt/Smith standard stars and on our SN-field comparison stars using the NOAO/DIGIPHOT/APPHOT package in IRAF (Tody 1993). The comparison stars were chosen so that they were reasonably well isolated and usually detected in all bands. A few sparse fields required also using stars that only had good detections in $B V R I / r^{\prime} i^{\prime}$ but not in $U$. An aperture with radius of 15 pixels was used on both the standard and comparison stars. An aperture correction was calculated from one or two bright, isolated, good curve-of-growth stars by subtracting the 6-pixel-radius-aperture magnitude from the 15-pixel-radius-aperture magnitude and applied to all of the stars in the field.

A photometric transformation solution for a given night was calculated from our Landolt/Smith stars using system of Equations (1). A linear dependence on air mass and color was sufficient for our intended level of final $V$-band comparison star precision $(\sim 0.015 \mathrm{mag})$. Higher-order terms were found to be consistent with zero and so we did not use them.

$$
\begin{aligned}
u-b & =z p_{u b}+\alpha_{u b} x+\beta_{u b}(U-B), \\
b-v & =z p_{b v}+\alpha_{b v} x+\beta_{b v}(B-V), \\
v-V & =z p_{v}+\alpha_{v} x+\beta_{v}(B-V) \\
v-r & =z p_{v r}+\alpha_{v r} x+\beta_{v r}(V-R), \\
v-i & =z p_{v i}+\alpha_{v i} x+\beta_{v i}(B-I) .
\end{aligned}
$$

The terms on the left side of the equations are the instrumental colors except for the $V$-band term. The first term on the right side of each equation is the zero point, followed by the air mass coefficients, $\alpha$, times the air mass, $x$. The $V$-band equation is unique in that it directly relates the instrumental magnitude $v$ to the standard system magnitude and color, $V$ and $B-V$. The other four equations only relate the instrumental and standard-system colors to each other. The final term on the right of the four color equations multiplies the standard-system color of the standard stars by a coefficient, $\beta$, to convert the standard-system color into the "calibrated" natural-system color.

Having solved for the zero point, air mass, and color coefficients by using the Landolt/Smith standards, this photometric solution was then applied to the comparison star measurements, producing tertiary standards that were used to calibrate the SN measurements.

Our goal was to observe each SN field on multiple photometric nights to ensure more accurate calibration. Sometimes this was not possible, but even in those cases $\mathrm{SN}$ fields that produced consistent, multiple-night calibration were observed on the same night, making us sufficiently confident that the SN fields with a single night of calibration were accurate. The uncertainties of the comparison stars include the measurement uncertainties, the standard deviation of measurements from multiple nights (for single nights, an appropriate error floor was used instead), and the uncertainty of the transformation to the standard system. The typical uncertainty of our $V$-band comparison star measurements is $0.015 \mathrm{mag}$. The average color coefficients are presented in Table 2.

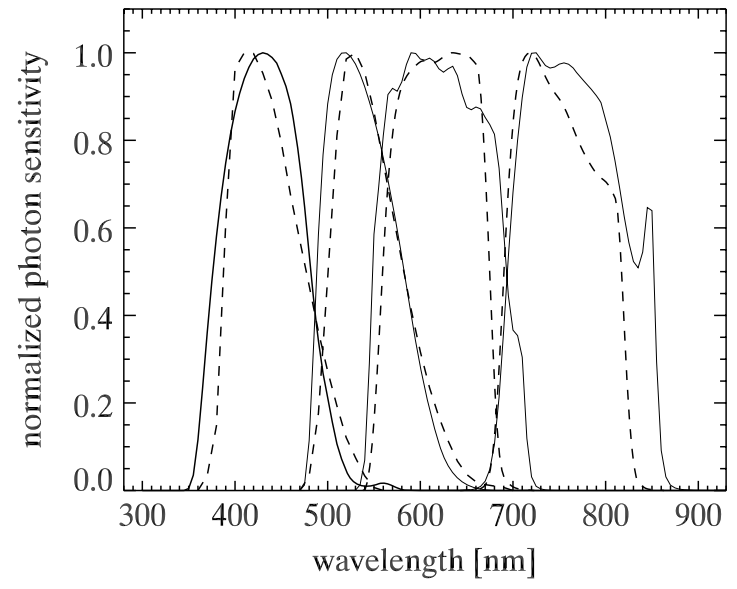

\begin{tabular}{|c|c|c|c|}
\hline Detector/Filters & Color Term & Value & Nights \\
\hline Keplercam/UBVr' $i^{\prime}$ & $(u-b) /(U-B)$ & $1.0279 \pm 0.0069$ & 20 \\
\hline Keplercam/UBV $V r^{\prime} i^{\prime}$ & $(b-v) /(B-V)$ & $0.9212 \pm 0.0029$ & 37 \\
\hline Keplercam $/ U B V r^{\prime} i^{\prime}$ & $(v-V) /(B-V)$ & $0.0185 \pm 0.0023$ & 37 \\
\hline Keplercam/UBVr$r^{\prime} i^{\prime}$ & $(v-r) /\left(V-r^{\prime}\right)$ & $1.0508 \pm 0.0029$ & 37 \\
\hline Keplercam $/ U B V r^{\prime} i^{\prime}$ & $(v-i) /\left(V-i^{\prime}\right)$ & $1.0185 \pm 0.0020$ & 37 \\
\hline Minicam/UBVr $r^{\prime} i^{\prime}$ & $(u-b) /(U-B)$ & $1.0060 \pm 0.0153$ & 4 \\
\hline Minicam $/ U B V r^{\prime} i^{\prime}$ & $(b-v) /(B-V)$ & $0.9000 \pm 0.0095$ & 4 \\
\hline Minicam $/ U B V r^{\prime} i^{\prime}$ & $(v-V) /(B-V)$ & $0.0380 \pm 0.0030$ & 4 \\
\hline Minicam/UBVr' $i^{\prime}$ & $(v-r) /\left(V-r^{\prime}\right)$ & $1.0903 \pm 0.0140$ & 4 \\
\hline Minicam $/ U B V r^{\prime} i^{\prime}$ & $(v-i) /\left(V-i^{\prime}\right)$ & $1.0375 \pm 0.0088$ & 4 \\
\hline 4Shooter/UBVRI & $(u-b) /(U-B)$ & $0.9912 \pm 0.0078$ & 16 \\
\hline 4Shooter/UBVRI & $(b-v) /(B-V)$ & $0.8928 \pm 0.0019$ & 16 \\
\hline 4Shooter/UBVRI & $(v-V) /(B-V)$ & $0.0336 \pm 0.0020$ & 16 \\
\hline 4Shooter/UBVRI & $(v-r) /(V-R)$ & $1.0855 \pm 0.0058$ & 16 \\
\hline 4Shooter/UBVRI & $(v-i) /(V-I)$ & $1.0166 \pm 0.0067$ & 16 \\
\hline
\end{tabular}

Figure 5. Synthesized natural system Keplercam $B V r^{\prime} i^{\prime}$ passbands (solid curves) with Bessell (1990) $B V$ and SDSS $r^{\prime} i^{\prime}$ overplotted (dashed curves).

Table 2

Photometric Color Terms

Notes. Lower-case $u b v r i$ refer to the instrumental magnitudes while $U B V R I r^{\prime} i^{\prime}$ refer to the standard magnitudes. All color terms implicitly contain an additive constant. For example, for the Keplercam: $(v-V)=0.0185(B-V)+$ const; $(u-b)=1.0279(U-B)+$ const.

We also synthesized natural system $B V r^{\prime} i^{\prime}$ passbands for the Keplercam by combining the primary and secondary mirror reflectivities (taken as the square of the measured reflectivity of the primary), the measured filter transmissions, and the measured Keplercam quantum efficiencies. No atmospheric component is included. We present these passbands as normalized photon sensitivities. A $U$-band filter transmission curve and the Minicam quantum efficiency were not available so passbands were not made for Keplercam $U$ or any of the Minicam bands. The 4Shooter BVRI passbands can be found in J06 as the "4Shooter/ Harris" combination and we point out that they are presented as normalized energy sensitivities. To convert to normalized photon sensitivities, the passbands should be divided by wavelength and renormalized. See Figure 5 for a visual representation of the Keplercam BVRI passbands. Our light curves were produced in the natural system and then converted to the standard system by using the color terms in Table 2 . The light curves and comparison stars, both natural and standard system versions, can be 
Table 3

Standard System Comparison Star Photometry

\begin{tabular}{lrlllllllllllllllll}
\hline \hline Star & R.A. (J2000) & Decl. (J2000) & $V$ & $d V$ & $N_{V}$ & $U-B$ & \pm & $N_{U}$ & $B-V$ & \pm & $N_{B}$ & $V-r^{\prime}$ & \pm & $N_{r^{\prime}}$ & $V-i^{\prime}$ & $p m$ & $N_{i^{\prime}}$ \\
\hline $2006 \mathrm{D}$ & & & & & & & & & & & & & & & & & & \\
01 & 125250.385 & -094237.78 & 17.684 & 0.037 & 2 & 1.325 & 0.219 & 2 & 1.058 & 0.061 & 2 & 0.572 & 0.061 & 2 & 1.119 & 0.067 & 2 \\
02 & 125246.717 & -095032.12 & 15.245 & 0.016 & 2 & 0.424 & 0.042 & 2 & 0.701 & 0.015 & 2 & 0.244 & 0.009 & 2 & 0.460 & 0.009 & 2 \\
03 & 125240.799 & -094843.84 & 16.548 & 0.017 & 2 & -0.029 & 0.051 & 2 & 0.469 & 0.016 & 2 & 0.164 & 0.016 & 2 & 0.316 & 0.013 & 2 \\
04 & 125238.144 & -094540.33 & 17.002 & 0.014 & 2 & 1.475 & 0.278 & 2 & 1.371 & 0.087 & 2 & 0.651 & 0.022 & 2 & 1.809 & 0.022 & 2 \\
05 & 125237.397 & -094212.02 & 16.492 & 0.022 & 2 & 0.047 & 0.057 & 2 & 0.521 & 0.018 & 2 & 0.179 & 0.014 & 2 & 0.355 & 0.010 & 2 \\
\hline
\end{tabular}

(This table is available in its entirety in a machine-readable form in the online journal. A portion is shown here for guidance regarding its form and content.)

Table 4

Natural (Instrumental) System Comparison Star Photometry

\begin{tabular}{|c|c|c|c|c|c|c|c|c|c|c|c|c|c|c|c|c|c|}
\hline Star & R.A. (J2000) & Decl. (J2000) & $V$ & $d V$ & $N_{V}$ & $U-B$ & \pm & $N_{U}$ & $B-V$ & \pm & $N_{B}$ & $V-r^{\prime}$ & \pm & $N_{r^{\prime}}$ & $V-i^{\prime}$ & $p m$ & $N_{i^{\prime}}$ \\
\hline \multicolumn{18}{|l|}{ 2006D } \\
\hline 01 & 125250.385 & -094237.78 & 17.7039 & 0.0375 & 2 & 1.3624 & 0.2186 & 2 & 1.0871 & 0.0610 & 2 & 0.5881 & 0.0611 & 2 & 1.1504 & 0.0668 & 2 \\
\hline 02 & 125246.717 & -095032.12 & 15.2575 & 0.0156 & 2 & 0.4356 & 0.0422 & 2 & 0.7210 & 0.0150 & 2 & 0.2511 & 0.0094 & 2 & 0.4724 & 0.0090 & 2 \\
\hline 03 & 125240.799 & -094843.84 & 16.5570 & 0.0166 & 2 & -0.0301 & 0.0510 & 2 & 0.4820 & 0.0156 & 2 & 0.1681 & 0.0163 & 2 & 0.3249 & 0.0125 & 2 \\
\hline 04 & 125238.144 & -094540.33 & 17.0275 & 0.0139 & 2 & 1.5162 & 0.2784 & 2 & 1.4091 & 0.0874 & 2 & 0.6696 & 0.0218 & 2 & 1.8589 & 0.0217 & 2 \\
\hline 05 & 125237.397 & -094212.02 & 16.5020 & 0.0220 & 2 & 0.0479 & 0.0573 & 2 & 0.5355 & 0.0182 & 2 & 0.1841 & 0.0139 & 2 & 0.3654 & 0.0098 & 2 \\
\hline
\end{tabular}

(This table is available in its entirety in a machine-readable form in the online journal. A portion is shown here for guidance regarding its form and content.)

Table 5

Standard System SN Light Curves

\begin{tabular}{lccc}
\hline \hline Filter & MJD & StdMag & dMag \\
\hline sn06D & & & \\
1 & 53753.51876 & 14.434 & 0.033 \\
1 & 53757.43733 & 14.224 & 0.025 \\
1 & 53764.45479 & 14.935 & 0.026 \\
1 & 53764.45843 & 14.962 & 0.026 \\
1 & 53765.43324 & 15.056 & 0.026 \\
1 & 53765.43685 & 15.048 & 0.025 \\
\hline
\end{tabular}

Notes. Filter codes: $U B V R I r^{\prime} i^{\prime}$ are 1, 2, 3, 4, 5, 13, and 14, respectively.

(This table is available in its entirety in a machine-readable form in the online journal. A portion is shown here for guidance regarding its form and content.)

found at our Web site ${ }^{34}$ and are archived with the journal. The Keplercam $B V r^{\prime} i^{\prime}$ passbands can also be found at these two locations. "Stub" examples of these tables are seen in Tables 3-7. The natural system passbands and photometry can be used together to avoid the uncertainty of using star-derived color terms but we do not pursue this here. Figure 6 shows nine of our better light curves.

\subsection{Internal Consistency Checks}

By choosing an appropriate shape for the PSF of the comparison stars we ensured accurate flux measurements for wellisolated stars. This also applies to cases where the SN is well isolated, allowing for two tests of the image-subtraction process: comparing the unsubtracted light curve with the light curve produced by subtracting a reference image taken with the same camera; doing the same procedure but with a reference image taken with a different camera. A third test involves comparing the light curve produced by subtracting a same-camera reference image with the light curve produced by subtracting a reference image

\footnotetext{
34 http://www.cfa.harvard.edu/supernova/CfA3
}

Table 6

Natural (Instrumental) System SN Light Curves

\begin{tabular}{lccc}
\hline \hline Filter & MJD & InstMag & dMag \\
\hline sn06D & & & \\
1 & 53753.51876 & 14.429 & 0.033 \\
1 & 53757.43733 & 14.216 & 0.025 \\
1 & 53764.45479 & 14.916 & 0.026 \\
1 & 53764.45843 & 14.944 & 0.026 \\
1 & 53765.43324 & 15.036 & 0.026 \\
1 & 53765.43685 & 15.027 & 0.025 \\
\hline
\end{tabular}

Notes. Filter codes: $U B V R I r^{\prime} i^{\prime}$ are 1, 2, 3, 4, 5, 13, and 14, respectively.

(This table is available in its entirety in a machine-readable form in the online journal. A portion is shown here for guidance regarding its form and content.)

Table 7

Keplercam $B V r^{\prime} i^{\prime}$ Passbands

\begin{tabular}{lc}
\hline \hline Wavelength $(\mathrm{nm})$ & Passband $(B$ band $)$ \\
\hline 345 & 0.000 \\
350 & 0.005 \\
355 & 0.035 \\
360 & 0.116 \\
365 & 0.234 \\
370 & 0.360 \\
\hline
\end{tabular}

Notes. Photon sensitivity passbands are the product of detector QE, filter transmission, primary mirror reflectivity, and secondary mirror reflectivity (approximated as the primary mirror reflectivity). No atmospheric term has been applied.

(This table is available in its entirety in a machine-readable form in the online journal. A portion is shown here for guidance regarding its form and content.)

from a different camera. As described below, we have done these tests and find internal consistency at about the $0.01 \mathrm{mag}$ level in most cases, when the $\mathrm{SN}$ is brighter than $17 \mathrm{mag}$. 

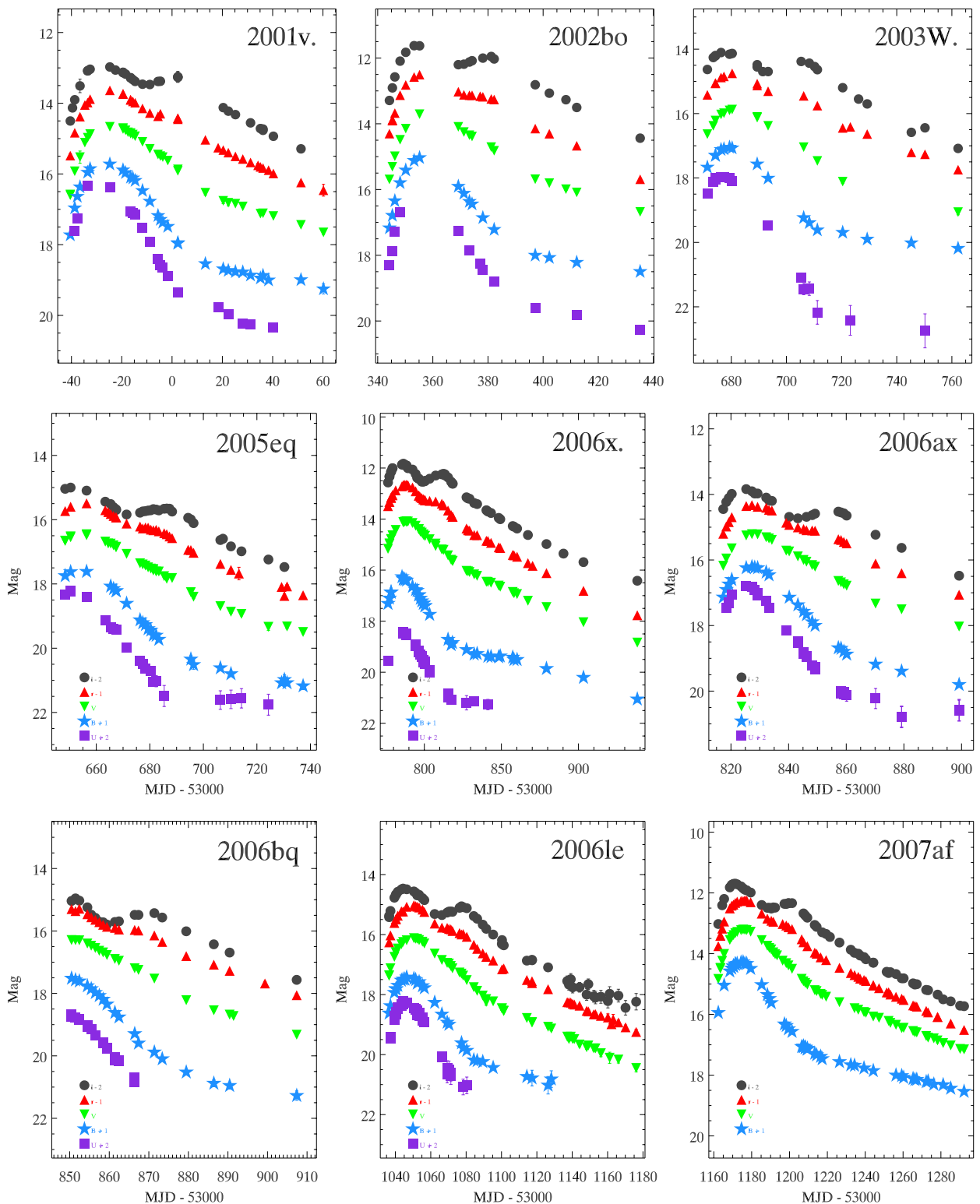

Figure 6. Nine of the better CfA3 SN Ia light curves. Error bars are smaller than the symbols in most cases. $U+2, B+1, V, R / r^{\prime}-1$, and $I / i^{\prime}-2$ have violet, blue, green, red, and black symbols, and are ordered from bottom to top in each plot.

(A color version of this figure is available in the online journal.)

\subsubsection{Same-Camera Subtracted Versus Unsubtracted Light Curves}

SN 2007af was very bright compared to its underlying galaxy background and the subtracted and unsubtracted light curves agree to better than 0.01 mag for most points, as seen in Figure 7, showing that the subtraction stage of the pipeline works well. The comparison plots also contain the weighted mean (WM) and $\chi^{2}\left(\mathrm{Chi}^{2}\right)$ of the differences.

SN 2006X allows two comparisons. Since it was fairly bright compared to its underlying galaxy light, the subtracted and unsubtracted light curves can be compared, especially in $i^{\prime}$, where dust extinction is the least and the PSF is the narrowest. Figure 8 shows that the unsubtracted light curve is slightly brighter at bright times, due to the small amount of underlying galaxy flux. Nonetheless, most points agree to better than 0.01 mag. At faint times, this galaxy flux becomes more significant. However, the agreement at bright times is a good indication that the subtraction stage is working well.
These two examples, plus several others that we do not present, show that the reference-image subtraction process itself does not introduce any significant offset into the final SN photometry.

\subsubsection{Cross-Camera Subtracted Versus Unsubtracted Light Curves}

Many of the 4Shooter and Minicam SN Ia reference images were acquired with the Keplercam and so it was important to see that the cross-camera subtraction works well. SN 2004et was a bright SN type II on a fairly smooth host-galaxy background. The SN data were taken on the Minicam while the reference image was acquired on the Keplercam. Figure 9 shows that the cross-camera subtracted and the unsubtracted $V$-band light curves agree within the uncertainties. At bright times, about twothirds of the points agree to better than $0.01 \mathrm{mag}$. The largest discrepancy is $0.03 \mathrm{mag}$. At faint times, the galaxy light begins to contribute more, and the unsubtracted light curve more is 


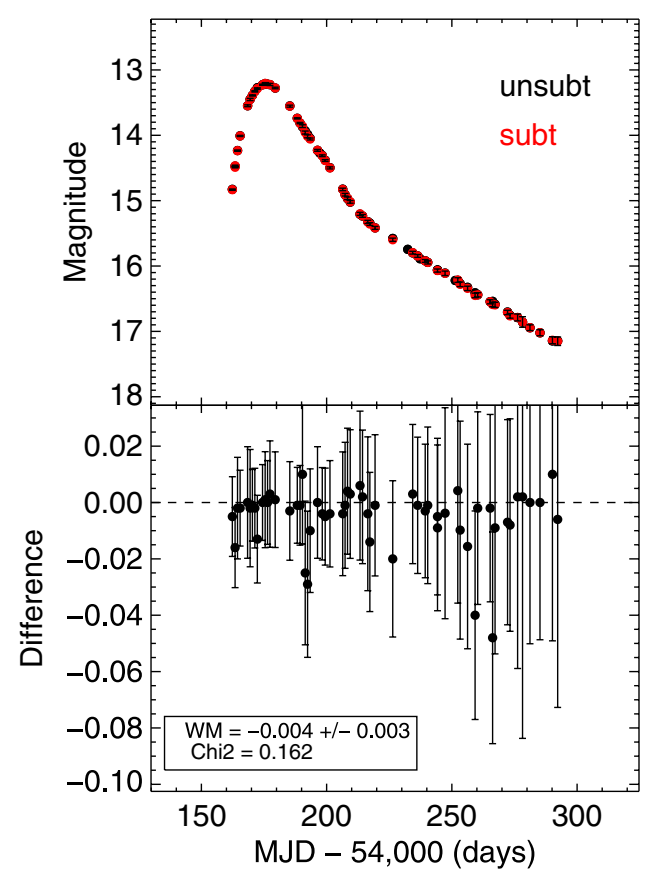

Figure 7. Comparison of the $V$-band unsubtracted and subtracted light curves of the bright SN 2007af. Most points agree to better than $0.01 \mathrm{mag}$. The weighted mean (WM) and $\chi^{2}$ of the differences are listed in the lower panel.

(A color version of this figure is available in the online journal.)

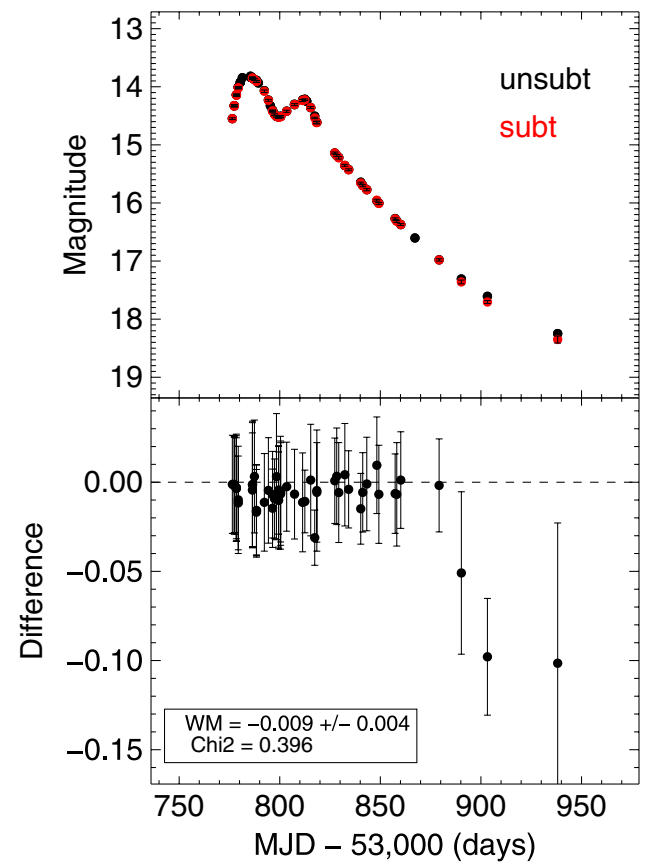

Figure 8. Comparison of the subtracted and unsubtracted $i^{\prime}$ light curves of the bright SN 2006X. Most points agree to better than $0.01 \mathrm{mag}$, suggesting that the reference-image subtraction is working well. The underlying galaxy flux only becomes evident at late times.

(A color version of this figure is available in the online journal.)

roughly 0.015 mag brighter with a scatter of $0.02 \mathrm{mag}$ but when the $\mathrm{SN}$ is bright, the cross-camera subtraction does not introduce any systematic error.

\subsubsection{Cross-Camera Subtraction Versus Same-Camera Subtraction}

The SN data for SN 2002jy were obtained with the 4Shooter while reference images were obtained with both the 4 Shooter

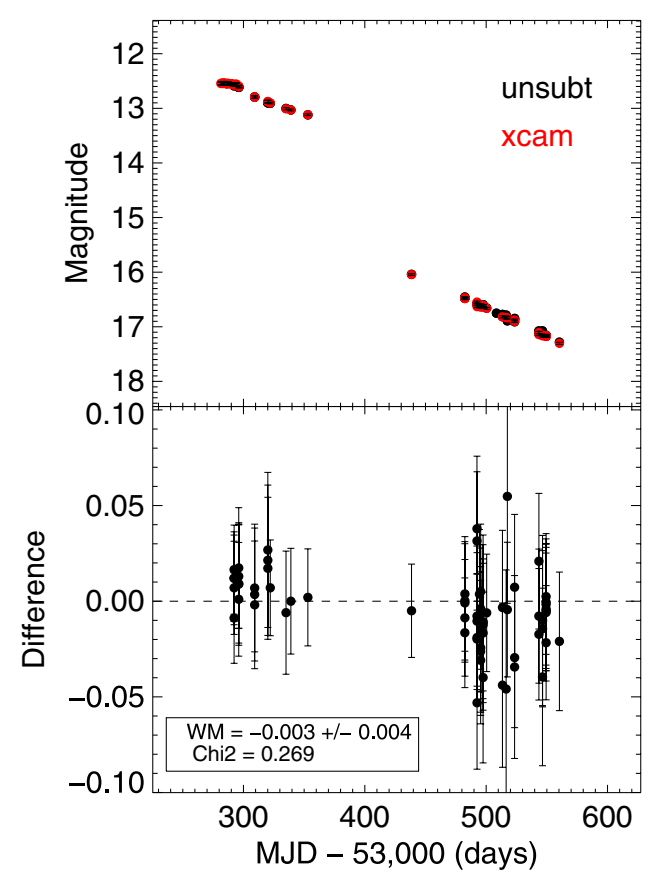

Figure 9. Comparison of the unsubtracted and cross-camera subtracted $V$-band light curves of SN 2004et.

(A color version of this figure is available in the online journal.)

and the Keplercam. There is excellent agreement between the BVRI same-camera and the cross-camera subtracted light curves, with typical agreement at the 0.01 mag level or better. The 4Shooter $U$-band reference image was of inferior quality and could not be used. The scatter is much smaller than the error bars because the only difference in the two light curves is the reference images, while the data images are the same. The $R$-band comparison is shown in Figure 10, with all the points agreeing to better than $0.01 \mathrm{mag}$. The slight differences in the light curves may be due to slight flux and seeing differences in the two reference images. Other factors include poorer 4 Shooter cosmetic properties and different responsivities between the cameras. We also found good agreement in other $\mathrm{SNe}$, bolstering our confidence that the cross-camera subtraction process was reliable.

\subsection{External Consistency Checks}

Comparisons with published photometry are made to check for consistency in comparison star calibration and SN Ia light curves. Differences in instruments, reduction techniques, and comparison star calibration are some of the factors leading to disagreements in the photometry from different telescopes of the same SN Ia. Typical disagreement of SN Ia photometry is roughly $0.02-0.05 \mathrm{mag}$ in $B V R$ around maximum light with larger discrepancies more common at later times and in $U$ and $I$ at all times. J06 present photometry comparisons from different groups for several SNe Ia and find typical agreement of several hundredths mag in most cases but worse in others.

SN 1999ee is an example where data was taken by two different telescopes on the same mountain and reduced in the exact same fashion with the same comparison star magnitudes (Stritzinger et al. 2002). The only difference was in the two telescopes/detectors. The differences in the two UBVRI light curves near maximum light were $-0.14,-0.01,-0.04,+0.04$, and -0.03 mag, respectively, and slightly larger a month later. $S$-corrections integrate the convolution of the natural system 


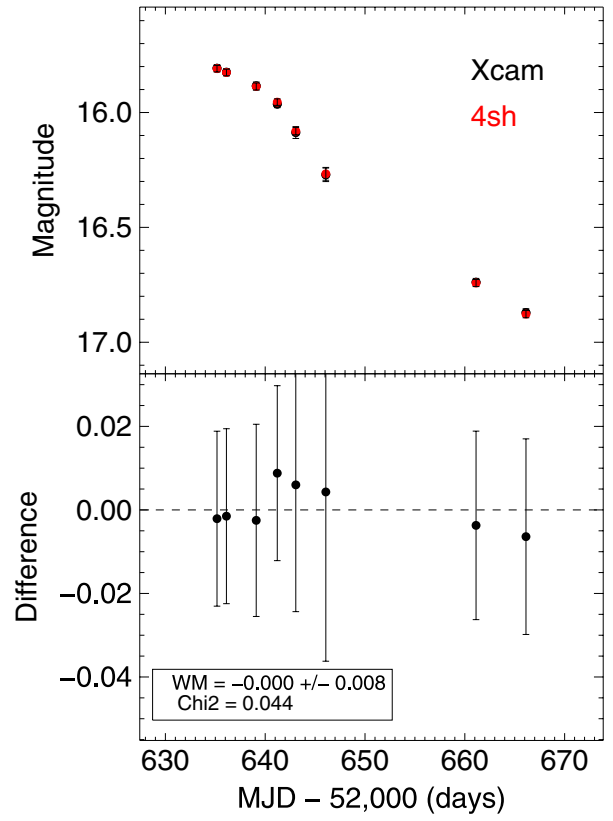

Figure 10. Comparison of the $R$-band same-camera subtracted and crosscamera subtracted light curves of SN 2002jy. The agreement is good, bolstering confidence that the cross-camera subtraction works reliably.

(A color version of this figure is available in the online journal.)

passband and SN spectrum and subtract the convolution of the standard system passband and SN spectrum. Because of the nonstellar spectra of SNe Ia, especially at later times, they can be used instead of star-derived color terms to more accurately place the SN photometry on the standard (or some other) system. $S$-corrections were applied, resulting in partial improvement for some bands and worsening in $R$, leading to the conclusion that accurate passbands must be determined if $S$-corrections are to be of use. Similarly to SN 1999ee, Suntzeff (2000) discusses the disagreement in the photometry of SN 1998bu from two telescopes that he reduced in the same manner with the same comparison stars. He finds a color difference between the two telescopes of $\delta(B-V)=0.12 \mathrm{mag}$ at late times, when the second telescope began observing. He finds that $S$-corrections would be able to correct this.

As another case, Krisciunas et al. (2003) applies $S$-corrections to SN 2001el. These are on the order of a few hundredths mag. Most $S$-corrections in the literature are roughly in the range 0.0 to $\pm 0.1 \mathrm{mag}$. In general, $S$-corrections can be large or small, depending on the mismatch between the natural system and standard system passbands and the spectral properties of the SN. SN 2005cf (Wang et al. 2009) is an example where the disagreement between different telescope's light curves is still 0.02-0.03 mag after $S$-corrections. This shows that differences of a few hundredths mag can occur even when many, but not all, of the systematic differences are not present and great care is taken in acquiring and processing the data.

As a check on our photometry pipeline, in Section 2.7.1, we first run the raw data of 17 SNe Ia from J06 through our photometry pipeline and compare the results. The J06 photometry pipeline mainly differs from the CfA3 pipeline in the reference image subtraction software. Then, for six objects from the literature, in Sections 2.7.2-2.7.7, we compare our CfA3 comparison stars and light curves with the published values. Of particular worth are the cases where values are presented from two or more telescopes. Overall, we find good consistency between our comparison star calibration and light curves in comparison to those from other groups for the same objects. This is of great importance when combining multiple data sets together to calculate dark energy properties. For purposes of comparing two sources of SN photometry, we define "excellent" agreement for all bands (except $U$ ) as better than 0.02 mag difference, "good" as 0.02-0.04 mag, "adequate" as 0.04-0.06, and "poor" as greater than 0.06 . For $U$, these values are doubled. These labels will be used in the following sections as a way of giving a measure of the agreement. Applying one of these labels to a light-curve comparison should be understood to apply to each point. For example, two light curves with excellent agreement would have no points disagreeing by more than 0.02 mag. Mostly good agreement would mean most of the points differ by no more than $0.04 \mathrm{mag}$. These labels are consistent with typical differences in published photometry of the same SN Ia from different groups, using different instruments (see above).

\subsubsection{Running J06 Data Through CfA3 Pipeline}

To test our photometry pipeline, we ran the 4Shooter $B V R I$ raw data for $17 \mathrm{SNe}$ Ia from J06 through our pipeline and compared the results with those obtained by J06, whose reduction methods differed from ours in some ways. The main difference was in the reference image subtraction software. J06 used the ISIS subtraction package (Alard \& Lupton 1998) as modified by B. Schmidt. We think that our more modern subtraction software is an improvement but this needs an empirical test. Another difference was our use of DoPHOT while J06 used aperture photometry. We note that we did not correct for fringing on the $I$-band images for these 17 objects (not to be confused with the fact that we did for the CfA3 I-band images). Running the $U$-band data through successfully requires more effort and does not add to determining the consistency of the J06 photometry pipeline with ours so we did not do it. The agreement was typically good or excellent around maximum light with typically good or adequate agreement at later times. A weighted mean of the difference between the two light curves was computed for each $\mathrm{SN}$ in each band. The average of these weighted means was $0.001 \pm 0.019 \mathrm{mag}$ for all bands while in each separate band they were $0.010 \pm 0.015 \mathrm{mag}$ in $B, 0.005 \pm 0.011 \mathrm{mag}$ in $V, 0.001 \pm 0.011 \mathrm{mag}$ in $R$, and $-0.012 \pm 0.029 \mathrm{mag}$ in $I$. The larger disagreement in $I$ is possibly due to our lack of fringe correction for these comparison objects. The two pipelines agree at $\lesssim 0.01 \mathrm{mag}$ in each band. These differences make clear the advantages of using one large sample that has been reduced by the same pipeline. It also illustrates the advantage that the CfA3 sample has since one of the significant high- $z$ samples, ESSENCE, uses the same pipeline. That each band's offset is consistent with zero is important since no significant, net offset is being introduced relative to the CfA 2 photometry, much of which was used to train the various light-curve fitters that are used in H09. We later will adopt 0.01 mag as the systematic uncertainty for our pipeline. As a representative example (neither the best nor the worst), we show the $B V$ light-curve comparisons of SN 1999gh in Figures 11 and 12.

\subsection{2. $S N 2003 d u$}

SN 2003du has four sets of photometry: CfA3 provides one and Stanishev et al. (2007), Leonard et al. (2005), and Anupama et al. (2005) provide the other three. We will refer to these as CfA3, STAN, LEO, and ANU. Our comparison stars agree to better than 0.02 mag with STAN in $B V I$, with LEO in $B V R I$, and with ANU in $I$. ANU is fainter in $B V$, by several 


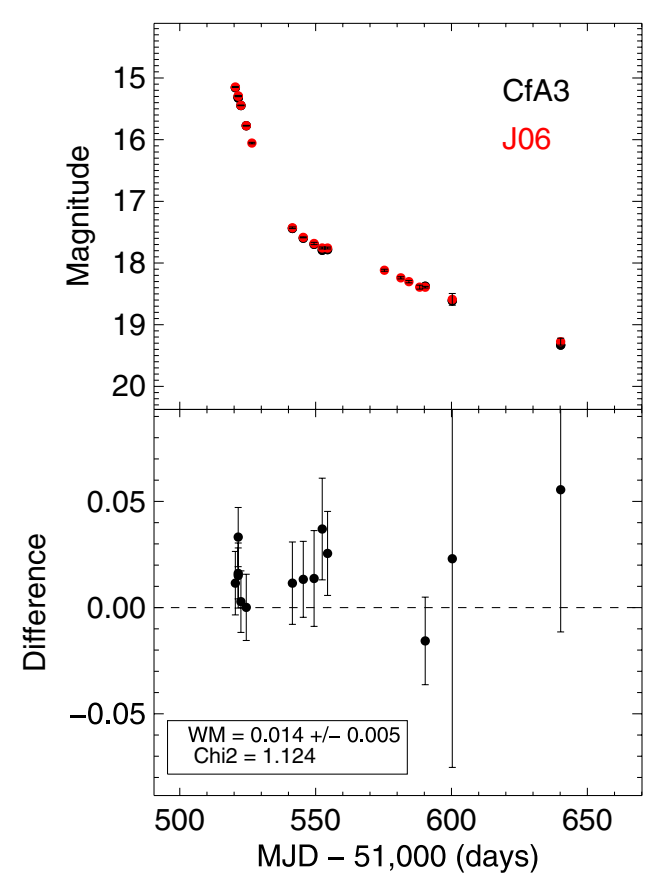

Figure 11. Comparison of the CfA3 and J06 versions of SN 1999gh in $B$. The slight offset and scatter is typical of the 17 SNe Ia from J06 that we ran through the CfA3 pipeline, showing that there is a slight difference between the two pipelines. However, there are both positive and negative offsets and sometimes both at different phases of the light curves of the 17 objects, suggesting that the pipelines are not introducing a definite positive or negative bias to all photometry. (A color version of this figure is available in the online journal.)

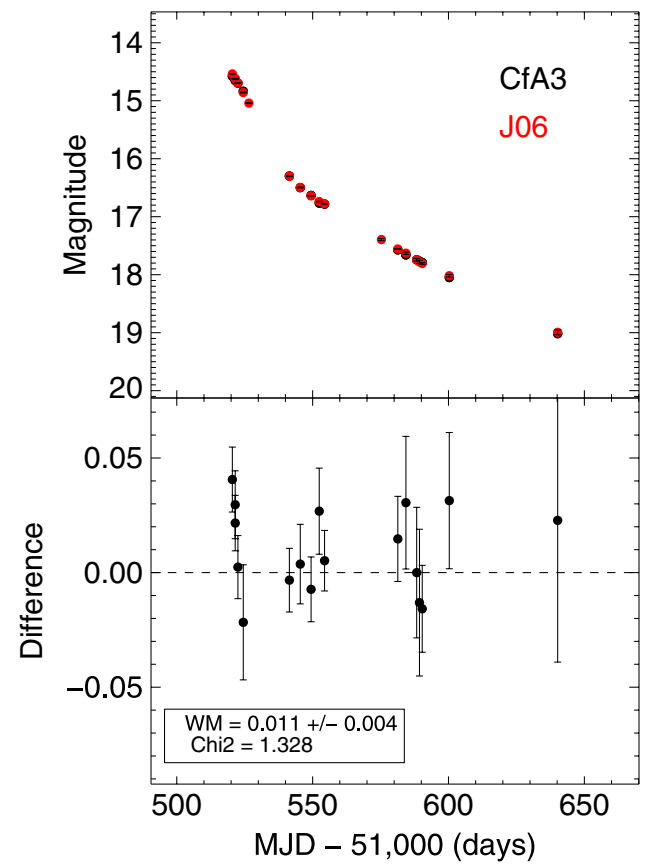

Figure 12. Comparison of the CfA3 and J06 versions of SN 1999gh in $V$, showing generally good agreement but with some scatter.

(A color version of this figure is available in the online journal.)

hundredths magnitude compared to the other three. STAN is the most discrepant in $R$. In $U$, CfA3 is fainter than STAN, but within the uncertainty. CfA3 pairings with the other groups are in as good or better agreement than most of the other non-CfA3 pairings.

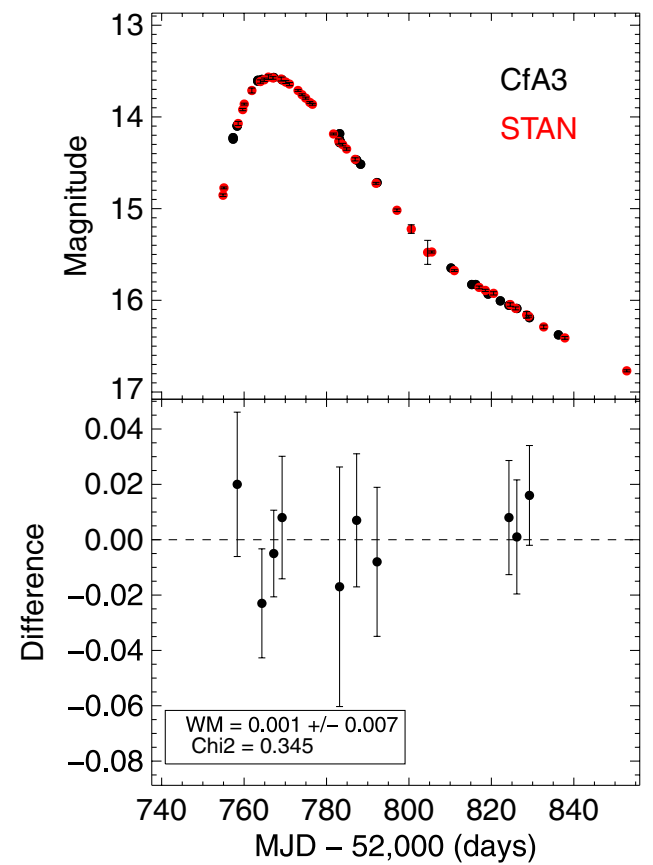

Figure 13. Comparison of the CfA3 and STAN $V$-band light curves of SN $2003 \mathrm{du}$, showing good agreement. This is a good sign since the STAN light curve comes from several telescopes and most of the points have been $S$-corrected while the CfA3 light curve is from one detector and has not been $S$-corrected.

(A color version of this figure is available in the online journal.)

The agreement of the CfA3 light curve is mostly excellent with STAN in $V$, as seen in Figure 13, and it is good in BRI, with a few points brighter by about $0.1 \mathrm{mag}$ at late times in $I$. STAN and LEO have good agreement in $R$ but STAN is systematically brighter in $B V$ by a few hundredths and in $I$ by 0.06 mag. CfA3 has good and excellent agreement with LEO in $R$ and $B$ but is brighter by about $0.03 \mathrm{mag}$ in $V$ and $0.07 \mathrm{mag}$ in $I$. ANU is fainter than CfA3 by around 0.04-0.05 mag in $B V$ around max, in rough agreement with the offset in the three comparison stars in common, but agrees better at late time. CfA3 and ANU have good agreement in RI. It is interesting to note that most of the STAN values we compare with were from various telescopes and $S$-corrected, suggesting that the CfA3 color-term transformations do a decent job of converting to the standard passbands.

\subsubsection{SN 2002bo}

Krisciunas et al. (2004b; KRIS) and Benetti et al. (2004; BEN) present optical light curves of SN 2002bo. In looking at the five comparison stars in common, those of KRIS are brighter than those of BEN by several hundredths mag. BEN only presents $B V R I$ data while CfA3 and KRIS also present $U$-band data. For the three comparison stars in common between KRIS and CfA3, excellent agreement is found in VRI, while KRIS is fainter by about $0.03 \mathrm{mag}$ in $B$ and $0.02 \mathrm{mag}$ in $U$, with a large scatter in $U$. CfA3 agrees excellently with BEN in $B$. CfA3 is brighter than BEN in VRI by 0.03 to 0.05 mag, but KRIS is even brighter, compared to BEN. Overall, in the bands presented by all three groups, CfA3 and KRIS agree best in VRI while CfA3 and BEN agree best in $B$.

KRIS presents both $S$-corrected light curves and color-termcorrected light curves. We find that our light curves agree slightly 
better with the $S$-corrected $B V$ light curves. The $B$ difference, CfA3-KRIS, is $0.026 \pm 0.021$ and in $V$ it is $0.004 \pm 0.031$. In $R$, there is little difference in which KRIS light curve we compare to. The agreement is good except for one poor point. In $I$, there is one KRIS point-their minimum point between the two I-band peaks - that seems excessively low compared to the shape of the neighboring points and so we consider this to be a bad point and not really worth comparing. It differs from ours by about 0.25 mag. Besides this point, our points around the first peak have good agreement with the $S$-corrected KRIS version and good-to-adequate agreement with the un- $S$ corrected version. There are two other KRIS $I$ points at later times where the un- $S$-corrected points are fainter than CfA3 by about $0.1 \mathrm{mag}$ but the (same) $S$-corrected points are only $\sim 0.02$ and 0.04 mag fainter. Finally, in $U$, both KRIS light curves are narrower and fainter than the CfA3 and there is very poor agreement-it is difficult to say which is right but the CfA3 light curve has a smoother shape and smaller error bars so it may be better.

KRIS and BEN generally have good-to-adequate agreement with a few poor points. The bad KRIS I-band point mentioned above is about 0.35 mag fainter than BEN, confirming that it is likely an aberrant point. Similarly, BEN and CfA3 have good-to-adequate agreement with a few poor points.

\subsubsection{SN $2002 b f$}

Leonard et al. (2005; LEO) present BVRI photometry of SN 2002 bf. We only have two comparison stars in common but they are consistent with zero difference in all bands except for one of the stars in I, where the LEO star is fainter by slightly more than the $1 \sigma$ level. As LEO note, the SN is only 4". 1 from its host galaxy's center, resulting in subtraction difficulties. As a result, both CfA3 and LEO light curves are somewhat choppy and several points have large error bars. The agreement in photometry is good to excellent in the two brightest $B$ points and in the brightest $V$ point, with adequate-to-poor agreement in most of the other $V$ points. The CfA3 light curves are mostly fainter in $R$, and in $B$ after the two brightest points. In $I$, there are some phases of good agreement and some of large disagreement ( $0.2 \mathrm{mag})$.

\subsubsection{SN $2005 h k$}

Phillips et al. (2007) present optical comparison star photometry and light curves for SN 2005hk from four different sources: CSP, CTIO, KAIT, and SDSS. We make no comparison with SDSS since their values are in $r i$ and not in $r^{\prime} i^{\prime}$. We compare the CfA3 comparison stars with the other telescopes' values where the stars and passbands are the same. In $B V$, CfA3, CSP, and CTIO all agree within $0.01 \mathrm{mag}$ in the mean while KAIT is most different from the other three but still in good-to-excellent agreement. In $U$, CfA3 and KAIT show the best agreement ( $0.01 \mathrm{mag})$ and CfA 3 and CTIO differ by $\sim 0.03$ mag. The CfA 3 comparison star photometry was calibrated on three nights, with excellent agreement, showing internal consistency. The excellent agreement with CSP and CTIO and the good-to-excellent agreement with KAIT suggest that our comparison star calibration is reliably on the standard system.

Phillips et al. (2007) compare the $B V$ KAIT and CSP light curves of SN 2005hk. The KAIT light curve is not referenceimage subtracted and this is probably the largest source of the discrepancies of several hundredths mag after $S$-correction. The
CSP light curve used a $g^{\prime}$ SDSS reference image for $B$ and a $g^{\prime}+r^{\prime}$ reference image for $V$ so this might be a small source of inaccuracy. They also compare the CSP and SDSS light curves in $r i$. $S$-corrections bring the two data sets into better agreement with some scatter at the level of a couple hundredths left over.

The CfA3 $V$ light curve shows good-to-excellent agreement, and excellent agreement in the mean, with CSP. The CfA3 $B$ light curve mostly shows good-to-excellent agreement with CSP, with a few poorly agreeing points, mostly at late times. The CfA $3 r^{\prime} i^{\prime}$ light curves are about 0.03 mag fainter, with a few poorly agreeing points at later times. Possible sources of disagreement are the reference images used, passband differences, and no $S$-corrections for the CfA3 light curves.

\subsubsection{SN 2005 am and SN $2005 c f$}

Li et al. (2006) present $U B V$ comparison star calibration of SN 2005am and 2005cf. Using the stars in common for SN 2005am, CfA3 is in excellent agreement with $\mathrm{Li}$ in the mean in $U(\sim 0 \mathrm{mag})$, but with a scatter of 0.08 mag. CfA3 is fainter by about 0.03 and $0.02 \mathrm{mag}$ in $B$ and $V$, respectively, but consistent with zero difference. No comparisons of the SN 2005am light curves are made since the Li light curve is not reference-image subtracted and the CfA3 light curve is. There are three $U B V$ comparison stars in common for SN 2005cf and there is excellent agreement in each band: less than $0.01 \mathrm{mag}$ mean difference for $B V$ and less than $0.025 \mathrm{mag}$ in $U$.

\subsubsection{SN $2006 X$}

We compare our $U B V$ comparison stars and light curves with those from Wang et al. (2008), who present data from KAIT, CTIO, and the Teramo-Normale Telescope (TNT). The comparison stars in common differ in $U B V$, respectively, as follows: $-0.043 \pm 0.116,0.008 \pm 0.041,-0.013 \pm 0.032$.

The CfA3 $B$-band light curve agrees excellently in the mean with the composite light curve from Wang et al. (2008) but several of the individual points are only in adequate or poor agreement. In $V$ band, CfA3 has excellent agreement with KAIT and CTIO in eight points, adequate in another, and poor $(0.07-$ $0.09 \mathrm{mag}$ ) in three more. TNT is systematically fainter than CfA 3 by about 0.06 mag. The TNT data are the most discrepant of any of the four groups.

\subsubsection{External Comparisons Summary}

Our external comparisons show that our light curves are consistent with those from other groups at about the same level that other groups' light curves are consistent with each other, typically at the "good" level of a few hundredths mag. This is important so that multiple nearby samples can be combined, both for training light-curve fitters and for calculating cosmological results. The reduction pipeline and the calibration to the standard system are usually the largest sources of systematic uncertainty between groups and so we emphasize the value of one large, homogeneously observed and reduced sample. The CfA3 sample fits the bill, with the slight exception of the three cameras used, and the change from RI filters on the 4Shooter to $r^{\prime} i^{\prime}$ on the later cameras. The CfA34Shooter subsample, with 64 objects, and the CfA3-Keplercam subsample, with 116 objects, each individually qualify as large, homogeneously observed and reduced samples since they were each observed with one telescope, one camera, and one set of filters. 


\subsection{Systematic Uncertainty}

The uncertainties of our comparison star photometry take into account both statistical uncertainty and the uncertainty in the photometric transformation and so no systematic error needs to be added. However, in calculating the differential photometry zero point to be added to the SN and its uncertainty (by calculating the weighted mean of the difference of the instrumental and calibrated magnitudes of the comparison stars) the individual star errors are treated as if they were purely statistical. The zero-point error is roughly equal to the typical individual comparison star uncertainty divided by the square root of the number of stars. This usually lowers the differential-photometry zero-point uncertainty below the amount of systematic uncertainty contained in the individual comparison star values and so our SN photometry uncertainties can be considered accurate in the natural system. A systematic uncertainty, on the order of the photometric transformation uncertainty (including photometric zero point and color term uncertainties), should be added when comparing CfA3 standard system SN photometry with that of other groups. We estimate this systematic uncertainty to be $0.02 \mathrm{mag}$ in $B V R I r^{\prime} i^{\prime}$ and 0.06 mag in $U$. This uncertainty was not added to the comparisons of literature and CfA3 SN Ia light curves above.

The other main source of systematic uncertainty for the CfA3 light curves comes from the photometry pipeline. The CfA3 photometry pipeline does propagate all the uncertainties of the various stages to the final $\mathrm{SN}$ measurement and are reliable in comparing with other CfA3 pipeline measurements. However, there may be differences compared to other photometry pipelines. Based on the differences in the CfA3 and J06 BVRI photometry of the 17 SNe Ia seen in Section 2.7.1 ( $\lesssim 0.01 \mathrm{mag}$ ) and an estimated reference-image flux zero-point uncertainty of $0.005 \mathrm{mag}$, we attribute a systematic uncertainty of $0.01 \mathrm{mag}$ to our pipeline photometry in $B V R I r^{\prime} i^{\prime}$. For $U$, we estimate this to be $0.02 \mathrm{mag}$.

Adding the photometric transformation and CfA3 pipeline systematics in quadrature gives a total systematic uncertainty of roughly $0.03 \mathrm{mag}$ in $B V R I r^{\prime} i^{\prime}$ and $0.07 \mathrm{mag}$ in $U$. The $U$ measurements should be used with caution. We emphasize that this level of systematic uncertainty is typical of the literature SN Ia photometry as well and has the potential to lead to systematic errors in derived cosmological quantities, such as the dark energy equation of state parameter, $w$. If there is a 0.03 mag offset from the true distance modulus in the nearby sample then this would roughly give rise to an error in $w$ of $\sim 0.06-$ 0.08 , roughly the same size as current statistical uncertainties in $w$ (H09). Using a homogeneously observed and reduced sample can reduce this systematic uncertainty.

As in H09, the "OLD" sample refers to the list of SNe Ia from Jha et al. (2007). For $0.01 \leqslant z_{\mathrm{CMB}} \leqslant 0.15$, the four different light-curve fitters, SALT, MLCS2k2 $\left(R_{V}=1.7\right)$, MLCS2k2 $\left(R_{V}=3.1\right)$, and SALT2, produce CfA3 Hubble residuals that are fainter on average than the OLD sample by $0.017 \pm$ $0.027,0.018 \pm 0.027,0.038 \pm 0.027$, and $0.056 \pm 0.032 \mathrm{mag}$, respectively. The average of these $(\sim 0.03 \pm 0.03 \mathrm{mag})$ is similar to our systematic uncertainty estimate, but consistent with no offset. Part of the difference in mean residuals is probably due to slightly different SN Ia populations being sampled, and how the light-curve/distance fitters interacts with them. Another part is possibly due to some sort of systematic difference in photometry. It should be noted that the standard deviation of the Hubble residuals is similar between the CfA3 and OLD samples,

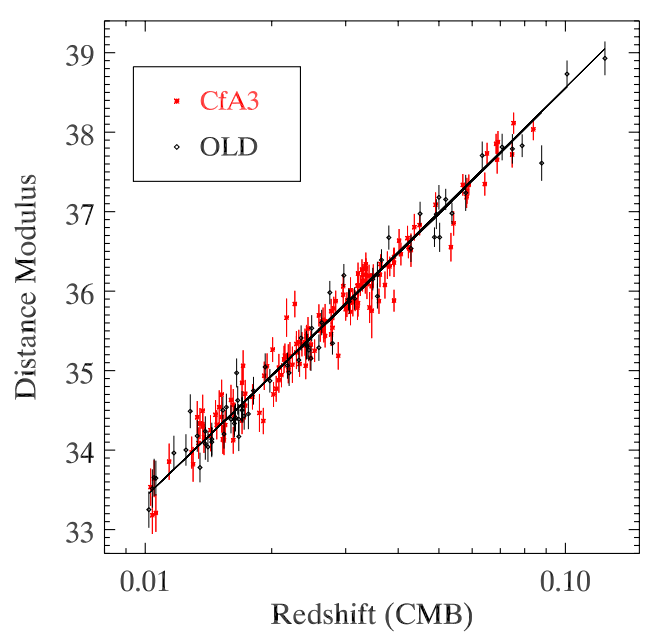

Figure 14. Hubble diagram of the CfA3 (red) and OLD (black) nearby SNe Ia. Distance moduli from H09 using MLCS2k2 $\left(R_{V}=1.7\right)$. The dispersion is 0.20 mag and the solid line is the distance modulus for a $\left(\Omega_{M}=0.27, \Omega_{\Lambda}=0.73\right)$ universe.

(A color version of this figure is available in the online journal.)

suggesting that the CfA3 photometry is about as intrinsically consistent as the OLD sample, although other effects contribute to this as well.

\section{SN I $a$ LIGHT-CURVE PROPERTIES}

We examine some of the SN Ia light-curve properties of the CfA3 and OLD samples. In H09, the CfA3 and OLD samples are fit with MLCS2k2, using the Galactic value of $R_{V}=3.1$ and $R_{V}=1.7$ (see Conley et al. 2007 for additional discussion). $R_{V}=3.1$ leads to an overestimate in the host extinction while $R_{V}=1.7$, chosen to remove the trend in Hubble residuals versus $A_{V}$ for the CfA3 sample, does not. We believe that $R_{V}=1.7$ MLCS2k2 extinction estimates are closer to reality so we choose here to use the light-curve shape parameter, $\Delta$, and the calculated host-galaxy extinction, $A_{V}$, from the $R_{V}=1.7$ MLCS2k 2 fits. A nearby Hubble diagram is presented in Figure 14 with the CfA3 points in red and the OLD points in black. This plot includes objects with a large range of extinction $\left(A_{V}<1.5\right)$ and lightcurve shape $(-0.4 \leqslant \Delta \leqslant 1.6)$, including the less-luminous, 1991 bg-like objects. The scatter for the CfA3+OLD sample is $0.20 \mathrm{mag}$, similar to the scatter of CfA3 and OLD separately.

We also take well sampled light curves from the CfA 3 and OLD samples that have data before maximum light in $B$ as measured by MLCS2k2 and measure properties directly from them, thus eliminating any dependency on a model or template light curve (see Tables 8 and 9). $K$-corrections are applied and Milky Way reddening (Schlegel et al. 1998) and time-dilation are removed, placing these in the rest frame but without any hostgalaxy reddening correction. A high-order polynomial (typically order 5 to provide enough flexibility to match the light-curve shape before and after maximum) is fit to the rest-frame $U B V$ light curves, smoothly passing through a large majority of the light-curve points, well inside the error bars, with only a few outliers in the more jagged light curves.

\subsection{UBV Decline Rates, $\Delta m_{15}$}

Where possible, the time of maximum and the magnitude at that time are measured in each of the $U B V$ bands from the polynomial fits just described. The $U$ and $V$ values are also measured at the time of $B$ maximum, where possible, to give 
Table 8

Direct-Fit and MLCS2k2 Light-Curve Properties

\begin{tabular}{|c|c|c|c|c|c|c|c|c|c|}
\hline SN & $z_{\mathrm{CMB}}$ & $m_{U}$ & $m_{B}$ & $m_{V}$ & $(B-V)_{\mathrm{B} \max }$ & $(U-B)_{\mathrm{B} \max }$ & $A_{V}$ & $E_{B V}$ & $E_{U B}$ \\
\hline $80 \mathrm{~N}$ & 0055 & $12.10(0.06)$ & $12.40(0.03)$ & $12.38(0.03)$ & $-0.02(0.05)$ & $-0.29(0.07)$ & $0.2110(0.0510)$ & $0.1241(0.0300)$ & $0.0881(0.0213$ \\
\hline 1B & & & & $1.89(0.05)$ & & & $.2300(0.0630)$ & & \\
\hline $81 \mathrm{D}$ & .0055 & $12.28(0.16)$ & $12.49(0.09)$ & $2.34(0.05)$ & & $-0.15(0.18)$ & $0.3390(0.1520)$ & $0.1994(0.0894)$ & $0.1416(0.0635)$ \\
\hline $86 \mathrm{G}$ & 0.0027 & $12.58(0.10)$ & $12.03(0.08)$ & & & $0.64(0.10)$ & $.2210(0.0860)$ & & \\
\hline 89B & 0.0035 & $12.20(0.07)$ & $12.23(0.09)$ & $11.88(0.05)$ & $0.32(0.10)$ & $0.04(0.11)$ & $0.8590(0.0810)$ & $0.5053(0.0476)$ & $0.3588(0.0338)$ \\
\hline $90 \mathrm{~N}$ & 0.0043 & $\ldots$ & $12.67(0.05)$ & $2.65(0.04)$ & $0.01(0.06)$ & $\ldots$ & $0.2210(0.0510)$ & $0.1300(0.0300)$ & $0.0923(0.0213)$ \\
\hline Oaf & 0.0502 & & $17.77(0.04)$ & $7.76(0.04)$ & $-0.01(0.06)$ & & $0.0730(0.0640)$ & $0.0429(0.0376)$ & $0.0305(0.0267)$ \\
\hline $91 \mathrm{~T}$ & 0.0069 & $11.16(0.03)$ & $11.60(0.02)$ & $11.45(0.02)$ & $0.12(0$ & $-0.45(0.04)$ & $0.3020(0.0390)$ & $0.1776(0.0229)$ & $0.1261(0.01$ \\
\hline $91 \mathrm{bg}$ & 0.0046 & & $14.60(0.05)$ & & & & & & \\
\hline $92 \mathrm{~A}$ & 0.0059 & $12.36(0.07)$ & $12.53(0 . \mathrm{C}$ & & & $-0.16(0.07)$ & & & \\
\hline $92 \mathrm{ag}$ & 0.0259 & & $16.20(0$. & & & & & & \\
\hline $92 \mathrm{al}$ & 0.0141 & & 14.45( & $14.55(($ & -0.10 & & & & \\
\hline $92 \mathrm{bc}$ & 0.0198 & & $15.08(C$ & & & & & & \\
\hline $92 \mathrm{bh}$ & 0.0451 & & & & & & & & \\
\hline $92 \mathrm{bo}$ & 0.0181 & & & & $-0.0^{\circ}$ & $\ldots$ & & & \\
\hline $92 \mathrm{bp}$ & & $\ldots$ & & & & $\ldots$ & & & \\
\hline $93 \mathrm{H}$ & 0.0248 & $\ldots$ & & & & $\ldots$ & & & \\
\hline 930 & 0.0519 & & & & & .. & & & \\
\hline $3 a g$ & 0.0500 & & & & & & & & \\
\hline $4 \mathrm{D}$ & 0.0031 & $11.14(0.09)$ & & & & $-0.62(0.10)$ & & & \\
\hline $94 \mathrm{~S}$ & 0.0160 & & & & & & & & \\
\hline $94 \mathrm{~T}$ & 0.03 & & & & & & & & \\
\hline $94 \mathrm{ae}$ & & & & & & & & & \\
\hline $95 \mathrm{D}$ & 0.00 & & & & -0.1 & & & & \\
\hline $95 \mathrm{E}$ & & & & & & & & & \\
\hline $5 \mathrm{ac}$ & & & & & & & & & \\
\hline 95ak & 0.0220 & & & & & $\ldots$ & & & \\
\hline $95 \mathrm{al}$ & & $12.72(0.10)$ & & & & $-0.57(0.11)$ & & & \\
\hline 95bd & 0.0144 & & & & & $\cdots$ & & & \\
\hline $96 \mathrm{X}$ & 0.0078 & $12.36(0.06)$ & & & & $-0.48(0.06)$ & & & \\
\hline 6 bo & 0.0163 & & & & & & & & \\
\hline $97 \mathrm{E}$ & 0.0133 & $14.77(0.10)$ & 15.1 & & & $-0.32(0.10)$ & & & \\
\hline $97 \mathrm{hn}$ & 0.0094 & $13.81(0.05)$ & & & & & & & \\
\hline & & & & & & & & & \\
\hline $97 \mathrm{dg}$ & & & & & & & & & \\
\hline 98aq & & 11.62( & & & & & & & \\
\hline $98 \mathrm{bp}$ & & $15.20(0$ & & & & -0.06 & & & \\
\hline $98 \mathrm{bu}$ & 0.00 & $11.78(0.04)$ & & & & $-0.29(0$ & & & \\
\hline 98de & 0.0156 & & & & & & & & \\
\hline 98es & 0.0096 & $13.26(0.06)$ & & & & $-0.54(0.06)$ & & & \\
\hline $9 \mathrm{aa}$ & & $14.17(0.06)$ & & & & -0.53 & & & \\
\hline $9 \mathrm{ac}$ & 0.0098 & $13.77(0.06)$ & & & & $-0.27(0.06)$ & & & \\
\hline $0_{0}$ & 0.0392 & & & & & & & & \\
\hline Ohy & 0.0028 & $13.73(0.02)$ & & & & $0.20(0.06)$ & & & \\
\hline $99 \mathrm{cc}$ & 0.0315 & $16.44(0.05)$ & & & & & & & \\
\hline $99 \mathrm{cl}$ & & $15.51(0.0$ & & & & $0.66(0$ & & & \\
\hline 99da & & & & & & 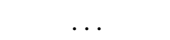 & & & \\
\hline $99 \mathrm{dk}$ & 0 & $14.54(0.09)$ & & & & 240 & & & \\
\hline $9 \mathrm{dq}$ & 0.0135 & $13.88(0.1$ & & & & -0.48( & & & \\
\hline $99 \mathrm{ee}$ & 0.0106 & $14.65(0.03)$ & & & & $-0.18(0$ & 0.6430 & 41) & 0.2685 \\
\hline 99ek & 0.0176 & & $15.61(0.37)$ & $15.49(0.28)$ & $0.10(0.37)$ & & $0.3120(0.1560)$ & $0.1835(0.0918)$ & $0.1303(0.0$ \\
\hline $99 \mathrm{gp}$ & 0.0260 & $15.40(0.06)$ & & $15.97(0.03)$ & $-0.00(0.05)$ & $-0.54(0.07)$ & & & \\
\hline $00 \mathrm{E}$ & 0.0042 & $\ldots$ & & & & & & & \\
\hline UCt & 0.0365 & & $17.08(0.0$ & & $-0.05(0$. & & & & \\
\hline $00 \mathrm{cn}$ & 0.0232 & $16.40(0.09)$ & & & & $-0.16(0.10)$ & $0.0710(0.0600)$ & $0.0418(0.0353)$ & \\
\hline 00dk & 0.0164 & $14.99(0.07)$ & & & $-0.02(0$. & $-0.29(0.07)$ & & $0.0100(0.0088)$ & \\
\hline $01 \mathrm{ba}$ & & & & & & & & & \\
\hline & & & & & & & & & \\
\hline $01 \mathrm{cz}$ & & & & & & $\ldots$ & & & \\
\hline 01el & & $12.56(0.04)$ & & & & $-016<0$ & & & \\
\hline 01ep & 0.0129 & $14.52(0.04)$ & & & & $-0.31(0.05)$ & & & \\
\hline 01fe & 0.0143 & $14.02(0.10)$ & $14.68(0.04)$ & $14.65(0.03)$ & $0.02(0.05)$ & $-0.60(0.09)$ & $0.0990(0.0490)$ & $0.0582(0.0288)$ & $0.0413(0.0204)$ \\
\hline 01fh & 0.0114 & $13.87(0.59)$ & $14.19(0.50)$ & $14.31(0.38)$ & $-0.15(0.50)$ & $-0.25(0.59)$ & $0.0770(0.0620)$ & $0.0453(0.0365)$ & $0.0322(0.0259)$ \\
\hline $01 \mathrm{~V}$ & 0.0162 & $14.01(0.09)$ & $14.64(0.08)$ & $14.61(0.05)$ & $-0.00(0.10)$ & $-0.60(0.12)$ & $0.1710(0.0410)$ & $0.1006(0.0241)$ & $0.0714(0.0171)$ \\
\hline $02 \mathrm{bo}$ & 0.0054 & $\ldots$ & $13.94(0.08)$ & $13.59(0.07)$ & $0.34(0.11)$ & $\ldots$ & $0.9080(0.0500)$ & $0.5341(0.0294)$ & $0.3792(0.0209)$ \\
\hline
\end{tabular}


Table 8

(Continued)

\begin{tabular}{|c|c|c|c|c|c|c|c|c|c|}
\hline SN & CMB & $m_{U}$ & $m_{B}$ & $m_{V}$ & $(B-V)_{\mathrm{Bmax}}$ & $(U-B)_{\mathrm{B} \max }$ & $A_{V}$ & $E_{B V}$ & $E_{U B}$ \\
\hline $02 \mathrm{~cd}$ & 0097 & $15.57(0.32)$ & $15.53(0.27)$ & $14.93(0.22)$ & $0.57(0.28)$ & $0.06(0.31)$ & $1.0260(0.1320)$ & $0.6035(0.0776)$ & $0.4285(0.0551)$ \\
\hline $02 \mathrm{cr}$ & & $\ldots$ & & & & & & & \\
\hline $02 \mathrm{cx}$ & 0250 & $\ldots$ & $7.54(0.10)$ & 7.34(0.08) & $0.17(0$. & & $.7030(0.0680)$ & $.4135(0.0400)$ & $0.2936(0.0284)$ \\
\hline 02de & 0281 & $16.32(0.06)$ & $6.66(0.03)$ & $6.52(0.02)$ & & $-0.31(0.06)$ & $.3820(0.0840)$ & $.2247(0.0494)$ & $0.1595(0.0351)$ \\
\hline $02 \mathrm{dj}$ & 0104 & $\ldots$ & $3.98(0.07)$ & $3.83(0.06)$ & & & $0.3420(0.0780)$ & $0.2012(0.0459)$ & $0.1429(0.0326)$ \\
\hline $02 \mathrm{dp}$ & 0105 & $14.16(0.06)$ & $4.60(0.05)$ & $4.47(0.05)$ & $0.10(0$. & $-0.39(0.07)$ & $0.2680(0.0900)$ & $0.1576(0.0529)$ & $0.1119(0.0376)$ \\
\hline $02 \mathrm{er}$ & 0.0085 & $13.91(0.13)$ & $14.24(0.11)$ & $14.10(0.09)$ & $0.12(0$. & $-0.33(0.13)$ & $0.2270(0.0740)$ & $0.1335(0.0435)$ & $0.0948(0.0309)$ \\
\hline 02fk & 0.0070 & & $3.11(0.05)$ & $13.23(0.04)$ & $-0.12(0$ & & & & \\
\hline $02 \mathrm{ha}$ & 0.0134 & & $4.69(0$ & & $-0.09(0$ & & $0.0420(0.0$ & & \\
\hline 02hu & 0.0382 & $16.08(0.05)$ & $16.58(C$ & 3) & -0.12( & $-0.48(0.06)$ & $0.0360(0$ & 76) & \\
\hline 03W & 0211 & $15.60(0.08)$ & & & & $-0.15(0.08)$ & & & \\
\hline $03 \mathrm{cg}$ & & $6.38(0$ & & & & 0.66 & & & \\
\hline 03du & & $13.07(0$. & & & -0.1 & -0.35 & 0.0320 & & \\
\hline 03iv & & $16.57(0$. & & & & -0.37( & & & \\
\hline $03 \mathrm{kf}$ & 0.0077 & $12.93(0.25)$ & & & & -0.27( & & & \\
\hline 04as & 0.0321 & $\ldots$ & & & & & & & \\
\hline $5 \mathrm{am}$ & 0095 & & & & & $-0.27(0.06)$ & & & \\
\hline $05 \mathrm{cf}$ & 0.0070 & $12.84(0.08)$ & 7) & & & -0.38 & & & \\
\hline $05 \mathrm{el}$ & 014 & $14.28(0$ & 8) & & -0.05 & -0.53( & & & \\
\hline $05 \mathrm{eq}$ & 0.028 & $15.77(0$. & & 16.25 & & $-0.47(0$ & & 76) & \\
\hline $05 \mathrm{eu}$ & 0.034 & & & & & & & & \\
\hline $05 \mathrm{hc}$ & 045 & $16.93(0.06)$ & $17.31(0.04)$ & & $-0.12(0$. & $-0.38(0$ & & & \\
\hline 05hk & & 5.410 & & & & 0.27 & & & \\
\hline $05 \mathrm{iq}$ & & & & & & -0.48 & & & \\
\hline $05 \mathrm{kc}$ & & & & & & -0.09 & & & \\
\hline & & & & & & & & & \\
\hline $5 \mathrm{ki}$ & & $14.96(0$ & & & & & & & \\
\hline $05 \mathrm{lz}$ & 040 & $17.33(0$ & & & & -0.22 & & & \\
\hline $5 \mathrm{mc}$ & 026 & $14(0)$ & & & & & & & \\
\hline $05 \mathrm{~ms}$ & 025 & $70 \%$ & 2) & 3) & -0.0 & -0.43 & & & \\
\hline $05 \mathrm{mz}$ & 0170 & & & & & & & & \\
\hline $06 a c$ & 0.0236 & $15.83(0.05)$ & & & & 0.25 & & & \\
\hline 06ar & 0.0229 & & & & & & & & \\
\hline 06ax & 0180 & $4.47(0.05$ & & & & $-0.50(0$. & & & \\
\hline 06az & 0 & $.87(0$. & & & & -0.52( & & & \\
\hline Sbr & & $\ldots$ & & & & & & & \\
\hline 06bt & & $\ldots$ & & & & & & & \\
\hline 06bz & & & & & & & & & \\
\hline $06 \mathrm{cc}$ & 0.0328 & $17.60(0.06)$ & & & & $-0.14(0.07)$ & & & \\
\hline $06 \mathrm{~cm}$ & 0.0152 & $\ldots$ & & & & & & & \\
\hline & 0 . & & & & & & & & \\
\hline & & $13.90(0.05)$ & & & & 02000 & & & \\
\hline & & & & & & & & & \\
\hline $06 \mathrm{gr}$ & 0.0335 & $16.59(0.09)$ & $16.91(0.07)$ & & $0.03(0.08)$ & $-0.28(0.10)$ & & & \\
\hline & 0.0208 & $\ldots$ & & & & & & & \\
\hline 06le & 017 & $14.26(0.32)$ & $178(027)$ & & & $-0.50(0.31)$ & & & \\
\hline 061f & 0 & $\ldots$ & & & & $\ldots$ & & & \\
\hline $06 \mathrm{mp}$ & 0 & $\ldots$ & & & & & & & \\
\hline $06 \mathrm{~N}^{1}$ & 0.014 & $\ldots$ & & & & $-0.43(0.08)$ & & & \\
\hline $06 \mathrm{nz}$ & & $\ldots$ & & & & & & & \\
\hline 06oa & & $17.46(0.08)$ & & & $-0.02(0.08)$ & $-0.30(0.10)$ & & & $0.0777(0.0280)$ \\
\hline $06 \mathrm{ob}$ & 0.0582 & $17.78(0.08)$ & $3.20(0.05)$ & $18.17(0.04)$ & $-0.02(0.06)$ & $-0.21(0.09)$ & & & $0.0088(0.0088)$ \\
\hline & 0.0300 & $\ldots$ & & & & & & & \\
\hline $06 \mathrm{~S}$ & 0.0329 & $16.34(0.05)$ & $16.79(0.0$ & $0.17(0$. & $-0.00(0.03)$ & $-0.43(0.05)$ & $0.2680(0.0460)$ & $0.1576(0.0271)$ & $0.1119(0.0192)$ \\
\hline $06 \mathrm{sr}$ & 0.0232 & & & & & & & & \\
\hline & & & & & & & & & \\
\hline & & $16.28(0.07)$ & & & & $1.00(0.09)$ & & & \\
\hline & & $\ldots$ & & & & & & & \\
\hline 07au & 0.0202 & $\ldots$ & & & & & & & \\
\hline $07 b c$ & & $\cdots$ & & & & & & & \\
\hline 07bd & 0.0320 & $\cdots$ & & & $-0.06(0.04)$ & & & & $0.0180(0.0138)$ \\
\hline $07 \mathrm{ca}$ & 0.0152 & $\ldots$ & $15.95(0.05)$ & $15.65(0.04)$ & $0.29(0.05)$ & & $0.5800(0.0690)$ & $0.3412(0.0406)$ & $0.2423(0.0288)$ \\
\hline $07 \mathrm{ci}$ & 0.0191 & $\ldots$ & & $15.86(0.02)$ & & & $0.0740(0.0630)$ & $0.0435(0.0371)$ & $0.0309(0.0263)$ \\
\hline $07 \mathrm{co}$ & 0.0266 & $16.39(0.10)$ & $16.43(0.08)$ & $16.38(0.06)$ & $0.03(0.08)$ & $-0.02(0.10)$ & $0.3920(0.0690)$ & $0.2306(0.0406)$ & $0.1637(0.0288)$ \\
\hline
\end{tabular}


Table 8

(Continued)

\begin{tabular}{lccrrrrrrr}
\hline \hline SN & $z_{\mathrm{CMB}}$ & $m_{U}$ & $m_{B}$ & $m_{V}$ & $(B-V)_{\mathrm{Bmax}}$ & $(U-B)_{\mathrm{Bmax}}$ & $A_{V}$ & $E_{B V}$ & $E_{U B}$ \\
\hline $07 \mathrm{cq}$ & 0.0247 & $\ldots$ & $15.82(0.07)$ & $15.79(0.06)$ & $0.01(0.08)$ & $\ldots$ & $0.1090(0.0590)$ & $0.0641(0.0347)$ & $0.0455(0.0246)$ \\
07F & 0.0242 & $\ldots$ & $15.87(0.03)$ & $15.91(0.02)$ & $-0.04(0.04)$ & $\ldots$ & $0.0470(0.0380)$ & $0.0276(0.0224)$ & $0.0196(0.0159)$ \\
07qe & 0.0244 & $\ldots$ & $16.01(0.04)$ & $15.99(0.03)$ & $-0.01(0.05)$ & $\ldots$ & $0.1480(0.0590)$ & $0.0871(0.0347)$ & $0.0618(0.0246)$ \\
07S & 0.0137 & $\ldots$ & $15.82(0.03)$ & $15.40(0.03)$ & $0.39(0.04)$ & $\ldots$ & $0.8330(0.0540)$ & $0.4900(0.0318)$ & $0.3479(0.0226)$ \\
08bf & 0.0253 & $15.29(0.08)$ & $15.72(0.04)$ & $15.68(0.04)$ & $0.04(0.05)$ & $-0.40(0.09)$ & $0.1020(0.0490)$ & $0.0600(0.0288)$ & $0.0426(0.0204)$ \\
\hline
\end{tabular}

Notes. The peak magnitudes, $m_{U}, m_{B}$, and $m_{V}$, are measured at the time of maximum light in each band while $(B-V)_{\mathrm{Bmax}}$ and $(U-B)_{\mathrm{Bmax}}$ are measured at the time of maximum light in $B$. For these measurements, the light curves were $K$-corrected, corrected for Milky Way extinction, and corrected for time dilation. The host-galaxy extinction, $A_{V}$, is from MLCS2k2 $\left(R_{V}=1.7\right)$ and has not been removed from the peak magnitudes listed. The host-galaxy color excesses, $E_{B V}$ and $E_{U B}$, are derived from $A_{V}$.

the color at maximum light. To measure the peak magnitude for a light curve in a given band, we require a point before and after maximum, with a separation of no more than $\Delta t=9.2$ days. Most of the light curves have much smaller separations but we want to measure the peak color for as many light curves as possible. We choose to use this wider separation limit because the peak magnitude calculations are fairly insensitive to the calculated time of maximum, as seen by removing points from light curves with many points around maximum and recalculating the peak magnitude. The uncertainty of each peak magnitude is taken to be the average of the uncertainties of the nearest points, one before and one after, plus $0.005 \Delta t$, added in quadrature.

We also measure the decline rate, $\Delta m_{15}$, in $U B V$ from the polynomial fits to the light curves. For this measurement, we also require that there be a point before and after $t=+15$ days, with a separation no greater than 12.2 days. The uncertainty in $\Delta m_{15}$ is approximated by summing (in quadrature) the uncertainty at peak and the uncertainty at $t=+15$ days. The magnitude at $t=+15$ days is sensitive to the time of maximum and we estimate its uncertainty to be the sum in quadrature of the average uncertainty of the surrounding points and the product of the slope of the light curve at $t=+15$ days times the uncertainty in $t_{\max }$. The slope of the light curve is roughly $0.1 \mathrm{mag}$ per day since a typical SN Ia declines $\sim 1$ mag in 15 days with most of that decline coming over the last 10 days. The uncertainty in $t_{\max }$ is estimated to be roughly 0.15 times the separation in time between the two surrounding points, determined by removing points from well sampled light curves and noting the effect.

Because we are mostly concerned with looking for trends in the plots, we choose to include the light curves with larger separations and lower precision measurements. For more precise purposes we advise the reader to have caution in using the peak magnitude and $\Delta m_{15}$ values derived from light curves with time separations between the two points near maximum of greater than 6 days. The time separations are listed in Table 9 for this purpose. We note that only $13.7 \%$ of the peak magnitudes and $12.5 \%$ of the $\Delta m_{15}$ values come from light curves with separations of more than 6 days.

\subsection{Decline Rates, Colors at Maximum Light, and $\Delta$}

In Figure 15, the 15 day decline rates in $U$ and $V$ are plotted against that of $B$. There is a fairly tight cluster of points, with a few outliers, in the bottom-left (slower decline) portion of the $V$-versus- $B$ panel with the fast decliners in $B$ showing a wide range of $V$ decline rates in the upper-right portion. The fastest $V$ decliners $\left(\Delta m_{15}(V)>1.2\right)$ are all 1991 bg-like SNe Ia (which can be identified as such by strong Ti II lines in their spectra). A linear relation between $B$ and $V$ decline rates cannot describe the data well. A quadratic relation does a better job. The upper panel shows a roughly linear relation between the $U$ and $B$ decline rates, with a larger amount of scatter than in the $V$-versus- $B$ comparison. This same qualitative effect was seen in the stretch relations of J06.

The $U-B$ and $B-V$ colors at $B$ maximum, directly measured from the light curves with Milky Way reddening correction but no host-galaxy reddening correction, are plotted in the top panel of Figure 16. In the bottom panel, they are corrected for the hostgalaxy reddening as measured by MLCS2k $2\left(R_{V}=1.7\right)$, and mostly form a tight cluster with $-0.2<(B-V)_{\max }<0.1$ and $-0.7<(U-B)_{\max }<0.0$. This behavior is similar to that seen in J06.

In Figure 17, the MLCS2k2 light-curve shape and luminosity parameter, $\Delta$, is plotted against the directly measured $\Delta m_{15}(B)$. There is a linear correlation for the slow and moderate decliners. The fast decliners have a wide range in $\Delta$.

In Figure 18, the host-galaxy corrected peak colors are plotted versus $\Delta$ and $\Delta m_{15}(B)$. Immediately standing out is the large range of red intrinsic $(B-V)_{\max }$ colors among the fast decliners, similar to what was found by Garnavich et al. (2004). The redder fast decliners are all 1991bg-like objects while many of the relatively bluer (though still red) fast decliners are the more intermediate $1986 \mathrm{G}$-like objects. The $(B-V)_{\max }$ versus $\Delta$ panel does suggest three interesting (though arbitrary) groupings of points. The slow and normal decliners form one group, with typical peak color of $(B-V)_{\max } \sim-0.1$. The moderately high- $\Delta$ objects, including $1986 \mathrm{G}$-like and some seemingly spectroscopically "normal" objects, form a second grouping with a typical color of 0.1 . Finally, the $1991 \mathrm{bg}$-like objects have a typical $(B-V)_{\max }$ color of 0.5 . Another way of grouping the objects in this plot is by their slope in color versus $\Delta$. Those with $\Delta<0.1$ have a flat slope while those with $0.1<\Delta<1.3$ have a slightly positive slope and those with $\Delta>1.4$ have a very steep slope. The $1991 \mathrm{bg}$-like objects are clearly distinct in $B-V$ color at maximum.

\subsection{Histograms of Color, Extinction, and $\Delta$}

Our intention is to use light-curve fitters that were trained on objects from the OLD sample, apply it to the CfA3 sample, and use the combined OLD+CfA3 sample to infer useful cosmological properties. To compare how similar the OLD and the CfA3 samples are, we take the nearby SN Ia with $z_{\mathrm{CMB}} \geqslant 0.01$. We plot a histogram of the directly measured, intrinsic peak color, $(B-V)_{\max }$, corrected for host reddening (see Figure 19). There are $44 \mathrm{CfA} 3$ and 48 Old objects and their distributions are fairly close-the two-sample KolmogorovSmirnov (KS) test gives a probability of $87 \%$ that they come from the same distribution. 
Table 9

$\Delta m_{15}, \Delta$, and Time Between Points at Maximum Light

\begin{tabular}{|c|c|c|c|c|c|c|c|}
\hline SN & $\Delta m_{15}(U)$ & $\Delta m_{15}(B)$ & $\Delta m_{15}(V)$ & $\Delta$ & $\Delta t(U)$ & $\Delta t(B)$ & $\Delta t(V)$ \\
\hline $80 \mathrm{~N}$ & & $1.28(0.05)$ & $0.73(0.05)$ & $-0.0400(0.0780)$ & 2.02 & 2.02 & 0.98 \\
\hline $81 B$ & $1.39(0.18)$ & $1.10(0.06)$ & $0.73(0.09)$ & $-0.1360(0.0700)$ & 0.66 & 0.36 & 3.50 \\
\hline 81D & $1.34(0.32)$ & $1.32(0.18)$ & $0.86(0.13)$ & $0.2900(0.2230)$ & 1.00 & 5.99 & 5.99 \\
\hline $86 \mathrm{G}$ & $2.05(0.24)$ & $1.65(0.09)$ & $1.01(0.09)$ & $1.2030(0.0640)$ & 5.09 & 0.99 & 3.94 \\
\hline $89 \mathrm{~B}$ & $1.24(0.10)$ & $1.02(0.13)$ & $0.64(0.19)$ & $0.0030(0.1150)$ & 0.99 & 3.99 & 3.99 \\
\hline $90 \mathrm{~N}$ & $\ldots$ & $1.04(0.13)$ & $0.62(0.11)$ & $-0.2990(0.0560)$ & $\ldots$ & 7.90 & 7.02 \\
\hline 90af & $\ldots$ & $1.63(0.06)$ & $0.89(0.07)$ & $0.5070(0.1190)$ & $\ldots$ & 0.99 & 1.90 \\
\hline $91 \mathrm{~T}$ & $1.37(0.04)$ & $0.80(0.03)$ & $0.62(0.05)$ & $-0.3510(0.0360)$ & 1.00 & 1.00 & 3.05 \\
\hline $91 \mathrm{bg}$ & $\ldots$ & $1.87(0.08)$ & $1.41(0.07)$ & $1.4350(0.0480)$ & $\ldots$ & 0.99 & 0.15 \\
\hline $92 \mathrm{~A}$ & $1.44(0.14)$ & $1.36(0.03)$ & $0.83(0.02)$ & $0.4130(0.0550)$ & 7.04 & 0.96 & 1.11 \\
\hline 92ag & $\ldots$ & $1.10(0.09)$ & $0.60(0.08)$ & $0.0500(0.0860)$ & $\ldots$ & 0.98 & 1.05 \\
\hline $92 \mathrm{al}$ & $\ldots$ & $1.10(0.08)$ & $0.61(0.08)$ & $-0.0640(0.0610)$ & $\ldots$ & 3.78 & 3.78 \\
\hline $92 b c$ & $\ldots$ & $0.82(0.08)$ & $0.61(0.08)$ & $-0.2530(0.0440)$ & $\ldots$ & 3.85 & 3.85 \\
\hline $92 \mathrm{bh}$ & $\ldots$ & $\ldots$ & $\ldots$ & $-0.1700(0.0860)$ & $\ldots$ & 2.81 & 2.81 \\
\hline $92 \mathrm{bo}$ & $\ldots$ & $\ldots$ & & $0.5800(0.0790)$ & $\ldots$ & 4.02 & 2.94 \\
\hline $92 \mathrm{bp}$ & $\ldots$ & $1.34(0.15)$ & $0.58(0.13)$ & $0.0090(0.0990)$ & $\ldots$ & 6.48 & 6.48 \\
\hline $93 \mathrm{H}$ & $\ldots$ & $1.76(0.09)$ & $1.02(0.07)$ & $0.8740(0.0960)$ & $\ldots$ & 1.11 & 0.87 \\
\hline 930 & $\cdots$ & $1.23(0.07)$ & $0.71(0.08)$ & $-0.0300(0.0720)$ & $\cdots$ & 2.13 & 3.61 \\
\hline 93ag & $\ldots$ & $\ldots$ & $\ldots$ & $-0.0190(0.0940)$ & $\ldots$ & 6.67 & 6.67 \\
\hline $94 \mathrm{D}$ & $1.71(0.12)$ & $1.35(0.07)$ & $0.81(0.05)$ & $0.3610(0.0490)$ & 0.81 & 0.65 & 0.49 \\
\hline $94 \mathrm{~S}$ & $\ldots$ & $\ldots$ & $0.68(0.09)$ & $-0.1730(0.0780)$ & $\ldots$ & 1.97 & 1.97 \\
\hline $94 \mathrm{~T}$ & $\ldots$ & $1.36(0.05)$ & $0.87(0.06)$ & $0.7460(0.1060)$ & $\ldots$ & 0.87 & 0.87 \\
\hline $94 \mathrm{ae}$ & $\ldots$ & $0.98(0.15)$ & $0.64(0.04)$ & $-0.2360(0.0440)$ & $\ldots$ & 7.96 & 0.99 \\
\hline 95D & $\ldots$ & $1.02(0.07)$ & $0.65(0.07)$ & $-0.2290(0.0480)$ & $\ldots$ & 1.98 & 2.02 \\
\hline $95 \mathrm{E}$ & $\ldots$ & $1.11(0.11)$ & $0.61(0.08)$ & $-0.0930(0.0660)$ & $\ldots$ & 2.87 & 2.87 \\
\hline $95 \mathrm{ac}$ & $\ldots$ & $0.77(0.07)$ & $0.58(0.08)$ & $-0.3160(0.0520)$ & $\ldots$ & 2.78 & 3.83 \\
\hline 95ak & $\ldots$ & & $0.86(0.07)$ & $0.1300(0.0800)$ & $\ldots$ & 1.01 & 0.86 \\
\hline $95 \mathrm{al}$ & $0.89(0.17)$ & $0.84(0.09)$ & $0.56(0.06)$ & $-0.2750(0.0490)$ & 3.97 & 3.98 & 1.00 \\
\hline $95 \mathrm{bd}$ & $\ldots$ & $0.94(0.34)$ & $0.74(0.25)$ & $-0.3270(0.0490)$ & $\ldots$ & 5.97 & 0.98 \\
\hline $96 \mathrm{X}$ & $1.37(0.10)$ & $1.29(0.06)$ & $0.81(0.04)$ & $0.0660(0.0560)$ & 0.99 & 1.09 & 1.00 \\
\hline $96 \mathrm{bo}$ & $\ldots$ & $1.23(0.06)$ & $0.70(0.07)$ & $-0.0350(0.0780)$ & $\ldots$ & 1.96 & 3.93 \\
\hline $97 \mathrm{E}$ & $1.64(0.11)$ & $1.41(0.09)$ & $0.79(0.09)$ & $0.3120(0.0860)$ & 1.07 & 1.86 & 3.94 \\
\hline $97 b p$ & $\ldots$ & $1.16(0.06)$ & $0.71(0.04)$ & $-0.2850(0.0560)$ & 1.00 & 0.98 & 2.05 \\
\hline $97 \mathrm{br}$ & $1.21(0.10)$ & $1.08(0.17)$ & $0.65(0.12)$ & $-0.3760(0.0390)$ & 1.90 & 6.79 & 5.96 \\
\hline $97 \mathrm{dg}$ & $\ldots$ & $\ldots$ & $\ldots$ & $-0.0180(0.0840)$ & 9.0 & 9.0 & 9.0 \\
\hline $98 \mathrm{aq}$ & $1.23(0.05)$ & $1.03(0.03)$ & $0.66(0.03)$ & $-0.1220(0.0380)$ & 2.01 & 1.03 & 0.94 \\
\hline $98 \mathrm{bp}$ & $2.36(0.10)$ & $1.96(0.08)$ & $1.12(0.04)$ & $1.2540(0.0470)$ & 2.98 & 2.98 & 1.04 \\
\hline $98 \mathrm{bu}$ & $1.16(0.05)$ & $1.04(0.03)$ & $0.75(0.03)$ & $-0.0660(0.0470)$ & 0.91 & 0.82 & 0.82 \\
\hline 98de & $\ldots$ & $1.99(0.12)$ & $1.27(0.06)$ & $1.5170(0.0420)$ & $\ldots$ & 0.98 & 1.02 \\
\hline 98es & $\ldots$ & $0.81(0.07)$ & $0.59(0.14)$ & $-0.3300(0.0370)$ & 1.03 & 0.89 & 7.99 \\
\hline 99 aа & $\ldots$ & $0.80(0.06)$ & $0.58(0.03)$ & $-0.3460(0.0320)$ & 4.82 & 2.02 & 0.86 \\
\hline $99 \mathrm{ac}$ & $\cdots$ & $1.33(0.06)$ & $\ldots$ & $-0.1440(0.0470)$ & 4.02 & 0.98 & 1.97 \\
\hline 99aw & $\ldots$ & $0.79(0.08)$ & $0.62(0.06)$ & $-0.4580(0.0420)$ & $\ldots$ & 4.72 & 1.18 \\
\hline 99by & $\ldots$ & $1.98(0.08)$ & $1.26(0.03)$ & $1.4650(0.0320)$ & 0.99 & 0.97 & 1.00 \\
\hline $99 \mathrm{cc}$ & $\ldots$ & $\ldots$ & $\ldots$ & $0.2880(0.0940)$ & 4.91 & 0.81 & 0.98 \\
\hline $99 \mathrm{cl}$ & $\ldots$ & $\ldots$ & $\ldots$ & $-0.0160(0.0860)$ & 1.98 & 1.95 & 2.99 \\
\hline 99da & $\ldots$ & $1.92(0.11)$ & $1.15(0.10)$ & $1.4870(0.0430)$ & $\ldots$ & 1.84 & 2.08 \\
\hline $99 \mathrm{dk}$ & $1.93(0.21)$ & $1.19(0.05)$ & $0.64(0.05)$ & $-0.3060(0.0530)$ & 7.89 & 1.24 & 1.24 \\
\hline $99 \mathrm{dq}$ & $\ldots$ & $0.86(0.11)$ & $0.55(0.08)$ & $-0.3590(0.0340)$ & 1.01 & 0.95 & 0.94 \\
\hline $99 \mathrm{ee}$ & $1.23(0.06)$ & $0.90(0.03)$ & $0.65(0.03)$ & $-0.2780(0.0370)$ & 2.94 & 0.95 & 0.87 \\
\hline $99 \mathrm{ek}$ & $\ldots$ & $1.21(0.37)$ & $0.72(0.28)$ & $0.0560(0.0780)$ & $\ldots$ & 2.03 & 0.85 \\
\hline $99 \mathrm{gp}$ & $1.05(0.08)$ & $0.80(0.07)$ & $0.55(0.04)$ & $-0.4150(0.0350)$ & 1.86 & 2.85 & 0.91 \\
\hline $00 \mathrm{E}$ & $\ldots$ & $0.94(0.25)$ & $0.66(0.19)$ & $-0.2270(0.0630)$ & $\ldots$ & 4.96 & 3.94 \\
\hline $00 \mathrm{cf}$ & $\cdots$ & $1.47(0.05)$ & $0.77(0.04)$ & $-0.0050(0.0790)$ & $\cdots$ & 1.13 & 1.13 \\
\hline $00 \mathrm{cn}$ & $\ldots$ & $\ldots$ & $\ldots$ & $0.7240(0.0840)$ & 5.95 & 5.95 & 0.92 \\
\hline $00 \mathrm{dk}$ & $1.86(0.08)$ & $\ldots$ & $\ldots$ & $0.5110(0.0660)$ & 0.91 & 1.84 & 4.91 \\
\hline $01 \mathrm{ba}$ & $\ldots$ & $0.95(0.05)$ & $0.59(0.07)$ & $-0.1640(0.0580)$ & $\ldots$ & 0.98 & 2.90 \\
\hline $01 \mathrm{bt}$ & $\ldots$ & $1.26(0.07)$ & $0.70(0.06)$ & $0.0410(0.0690)$ & $\ldots$ & 2.83 & 3.01 \\
\hline $01 \mathrm{cz}$ & $\ldots$ & $0.93(0.08)$ & $0.62(0.06)$ & $-0.1240(0.0610)$ & $\ldots$ & 2.89 & 1.97 \\
\hline 01el & $1.55(0.07)$ & $1.15(0.07)$ & $0.63(0.02)$ & $-0.1150(0.0530)$ & 4.03 & 4.02 & 1.02 \\
\hline 01ep & $\ldots$ & $\ldots$ & $\ldots$ & $0.0460(0.0810)$ & 0.84 & 3.97 & 3.98 \\
\hline $01 \mathrm{fe}$ & $\ldots$ & $\ldots$ & $\ldots$ & $-0.1690(0.0670)$ & 6.88 & 5.88 & 4.03 \\
\hline 01fh & $\ldots$ & $\ldots$ & $\ldots$ & $0.6320(0.1240)$ & 0.97 & 3.01 & 2.93 \\
\hline $01 \mathrm{~V}$ & $1.01(0.18)$ & $0.65(0.15)$ & $0.53(0.13)$ & $-0.3300(0.0430)$ & 8.70 & 7.74 & 7.74 \\
\hline
\end{tabular}


Table 9

(Continued)

\begin{tabular}{|c|c|c|c|c|c|c|c|}
\hline SN & $\Delta m_{15}(U)$ & $\Delta m_{15}(B)$ & $\Delta m_{15}(V)$ & $\Delta$ & $\Delta t(U)$ & $\Delta t(B)$ & $\Delta t(V)$ \\
\hline 02bo & & & & $-0.1060(0.0640)$ & & & - \\
\hline $02 \mathrm{~cd}$ & $1.06(0.33)$ & $0.98(0.27)$ & $\ldots$ & $-0.3210(0.0560)$ & 3.95 & 3.96 & $\ldots$ \\
\hline $02 \mathrm{cr}$ & $\ldots$ & $1.26(0.11)$ & $0.66(0.11)$ & $0.0090(0.0830)$ & $\ldots$ & 6.83 & 6.82 \\
\hline $02 \mathrm{cx}$ & $\ldots$ & $1.32(0.14)$ & $0.79(0.13)$ & $-0.5320(0.0580)$ & $\ldots$ & 5.86 & 6.87 \\
\hline $02 \mathrm{de}$ & $\ldots$ & $\ldots$ & $\ldots$ & $-0.2240(0.1430)$ & 2.00 & 2.94 & 2.94 \\
\hline $02 \mathrm{dj}$ & $\ldots$ & $\ldots$ & $\ldots$ & $-0.2000(0.1200)$ & $\ldots$ & 1.96 & 7.94 \\
\hline $02 \mathrm{dp}$ & $\ldots$ & $1.12(0.06)$ & $\ldots$ & $0.0230(0.1350)$ & 1.97 & 1.97 & 6.86 \\
\hline $02 \mathrm{er}$ & $1.87(0.13)$ & $1.28(0.12)$ & $0.73(0.10)$ & $0.2700(0.0780)$ & 0.99 & 1.03 & 1.94 \\
\hline $02 \mathrm{fk}$ & $\ldots$ & $\ldots$ & $0.65(0.10)$ & $-0.0620(0.0560)$ & $\ldots$ & 6.98 & 6.02 \\
\hline 02ha & $\ldots$ & $1.34(0.15)$ & $0.78(0.15)$ & $0.1270(0.0790)$ & $\ldots$ & 8.84 & 8.84 \\
\hline 02hu & $1.32(0.09)$ & $1.04(0.07)$ & $0.53(0.07)$ & $-0.2460(0.0550)$ & 3.84 & 3.85 & 3.84 \\
\hline 03W & $1.35(0.10)$ & $1.16(0.04)$ & $0.71(0.14)$ & $-0.0710(0.0590)$ & 1.04 & 0.94 & 8.90 \\
\hline $03 \mathrm{cg}$ & $\ldots$ & $\ldots$ & $0.68(0.05)$ & $0.0230(0.0740)$ & 3.08 & 5.91 & 2.84 \\
\hline 03du & $\ldots$ & $\ldots$ & $\ldots$ & $-0.1680(0.0450)$ & 6.94 & 2.86 & 2.86 \\
\hline 03iv & $\ldots$ & $\ldots$ & $\ldots$ & $0.2780(0.1060)$ & $\ldots$ & 1.97 & 4.80 \\
\hline $03 \mathrm{kf}$ & $0.94(0.28)$ & $0.97(0.23)$ & $0.70(0.19)$ & $-0.1710(0.0550)$ & 9.02 & 6.10 & 6.11 \\
\hline 04as & $\ldots$ & ... & $0.64(0.06)$ & $-0.1840(0.0700)$ & $\ldots$ & $\ldots$ & 2.81 \\
\hline 05am & $\ldots$ & $1.73(0.05)$ & $0.89(0.05)$ & $0.4000(0.0910)$ & $\ldots$ & 1.01 & 0.95 \\
\hline $05 \mathrm{cf}$ & $1.30(0.09)$ & $1.06(0.08)$ & $0.61(0.06)$ & $-0.1470(0.0810)$ & 3.05 & 3.03 & 1.00 \\
\hline $05 \mathrm{el}$ & $1.65(0.17)$ & $1.23(0.11)$ & $0.79(0.10)$ & $0.2100(0.0600)$ & 8.84 & 4.85 & 4.85 \\
\hline 05 eq & $1.18(0.13)$ & $0.86(0.11)$ & $0.49(0.10)$ & $-0.3090(0.0450)$ & 5.78 & 5.79 & 5.78 \\
\hline $05 \mathrm{eu}$ & $\ldots$ & $\ldots$ & $0.67(0.15)$ & $-0.3190(0.0560)$ & $\ldots$ & $\ldots$ & 7.89 \\
\hline $05 \mathrm{hc}$ & $1.49(0.15)$ & $0.97(0.05)$ & $0.53(0.10)$ & $-0.1250(0.0750)$ & 1.04 & 0.86 & 5.69 \\
\hline 05hk & $1.72(0.06)$ & $1.47(0.14)$ & $0.83(0.03)$ & $-0.3100(0.0460)$ & 0.88 & 8.87 & 0.91 \\
\hline $05 \mathrm{iq}$ & $1.88(0.31)$ & $1.05(0.10)$ & $0.68(0.04)$ & $0.1370(0.0730)$ & 5.84 & 4.72 & 0.89 \\
\hline $05 \mathrm{kc}$ & $\ldots$ & $1.24(0.19)$ & $0.66(0.14)$ & $0.0360(0.0820)$ & 2.88 & 1.93 & 1.93 \\
\hline $05 \mathrm{ke}$ & $1.77(0.06)$ & $1.66(0.14)$ & $1.15(0.13)$ & $1.5510(0.0330)$ & 0.01 & 8.86 & 7.86 \\
\hline 05ki & $\ldots$ & $\ldots$ & $\ldots$ & $0.2850(0.0660)$ & 8.80 & 8.80 & 1.02 \\
\hline $051 \mathrm{lz}$ & $\ldots$ & $1.35(0.19)$ & $0.59(0.09)$ & $0.2170(0.1030)$ & 3.02 & 3.02 & 3.02 \\
\hline $05 \mathrm{mc}$ & $1.96(0.36)$ & $1.87(0.11)$ & $1.04(0.07)$ & $0.9350(0.0710)$ & 1.02 & 3.92 & 3.91 \\
\hline $05 \mathrm{~ms}$ & $\ldots$ & $0.79(0.07)$ & $0.56(0.04)$ & $-0.1590(0.0520)$ & 3.90 & 3.91 & 1.83 \\
\hline $05 \mathrm{mz}$ & $\ldots$ & $1.96(0.14)$ & $1.33(0.11)$ & $1.3640(0.0670)$ & 1.98 & 1.94 & 3.97 \\
\hline $06 \mathrm{ac}$ & $1.46(0.08)$ & $1.08(0.07)$ & $0.66(0.08)$ & $0.1610(0.0790)$ & 1.91 & 3.86 & 4.97 \\
\hline 06ar & .. & $\ldots$ & .. & $0.4280(0.2430)$ & $\ldots$ & 3.93 & 2.85 \\
\hline $06 a x$ & $1.39(0.10)$ & $1.08(0.05)$ & $0.63(0.05)$ & $-0.1620(0.0480)$ & 4.93 & 1.95 & 1.94 \\
\hline 06az & $1.52(0.11)$ & $1.30(0.06)$ & $0.73(0.05)$ & $0.1540(0.0560)$ & 4.83 & 2.08 & 1.91 \\
\hline 06br & $\ldots$ & $1.47(0.20)$ & $0.89(0.08)$ & $0.0450(0.1500)$ & $\ldots$ & 0.89 & 0.90 \\
\hline 06bt & $\ldots$ & $1.09(0.06)$ & $0.54(0.04)$ & $-0.3250(0.0520)$ & $\ldots$ & 1.04 & 0.98 \\
\hline $06 \mathrm{bz}$ & $\ldots$ & $2.09(0.16)$ & $1.41(0.06)$ & $1.5020(0.0820)$ & $\ldots$ & 0.92 & 1.08 \\
\hline $06 \mathrm{cc}$ & $1.07(0.15)$ & $1.01(0.05)$ & $0.72(0.06)$ & $-0.2260(0.0580)$ & 3.75 & 0.96 & 2.81 \\
\hline $06 \mathrm{~cm}$ & $\ldots$ & $0.99(0.13)$ & $0.79(0.07)$ & $-0.0520(0.0870)$ & $\ldots$ & 0.94 & 1.97 \\
\hline $06 \mathrm{cp}$ & $\ldots$ & $\ldots$ & & $-0.1720(0.0870)$ & $\ldots$ & 4.84 & 4.84 \\
\hline $06 \mathrm{D}$ & $1.85(0.08)$ & $1.35(0.07)$ & $0.84(0.11)$ & $0.4230(0.0770)$ & 3.88 & 3.88 & 6.96 \\
\hline $06 \mathrm{gj}$ & $\ldots$ & $1.39(0.17)$ & $0.96(0.15)$ & $0.5820(0.1590)$ & $\ldots$ & 8.71 & 8.71 \\
\hline $06 \mathrm{gr}$ & $\ldots$ & $0.95(0.13)$ & $0.57(0.08)$ & $-0.3050(0.0460)$ & 1.85 & 6.91 & 3.93 \\
\hline $06 \mathrm{kf}$ & $\ldots$ & $\ldots$ & $0.77(0.12)$ & $0.6280(0.0970)$ & $\ldots$ & $\ldots$ & 0.87 \\
\hline 06le & $1.04(0.33)$ & $0.85(0.27)$ & $0.59(0.22)$ & $-0.2720(0.0440)$ & 2.01 & 4.02 & 4.03 \\
\hline 06lf & $\ldots$ & $1.35(0.62)$ & $0.71(0.49)$ & $0.2920(0.0790)$ & $\ldots$ & 6.06 & 6.06 \\
\hline $06 \mathrm{mp}$ & $\ldots$ & $\ldots$ & $\ldots$ & $-0.1210(0.0580)$ & $\ldots$ & 1.92 & 5.87 \\
\hline $06 \mathrm{~N}$ & $\ldots$ & $1.57(0.07)$ & $0.90(0.06)$ & $0.4230(0.0700)$ & $\ldots$ & 1.85 & 1.85 \\
\hline $06 n z$ & $\ldots$ & $\ldots$ & $1.18(0.14)$ & $1.1150(0.1180)$ & $\ldots$ & 7.58 & 7.57 \\
\hline $060 \mathrm{a}$ & $\ldots$ & $0.98(0.18)$ & $0.60(0.14)$ & $-0.2520(0.1000)$ & 0.93 & 5.67 & 3.77 \\
\hline $06 \mathrm{ob}$ & $\ldots$ & $1.70(0.12)$ & $1.15(0.11)$ & $0.5410(0.0780)$ & 1.03 & 1.80 & 1.94 \\
\hline $06 \mathrm{qo}$ & $\cdots$ & $1.02(0.10)$ & $0.61(0.10)$ & $-0.1820(0.0550)$ & $\ldots$ & 5.74 & 5.75 \\
\hline $06 \mathrm{~S}$ & $\ldots$ & $0.91(0.04)$ & $0.60(0.05)$ & $-0.1980(0.0530)$ & 2.01 & 0.93 & 2.82 \\
\hline $06 s r$ & $\ldots$ & $1.26(0.09)$ & $0.72(0.08)$ & $0.1870(0.0860)$ & $\ldots$ & 3.87 & 3.88 \\
\hline 06td & $\ldots$ & $1.48(0.12)$ & $0.76(0.10)$ & $0.3900(0.1380)$ & $\ldots$ & 0.99 & 0.98 \\
\hline $06 \mathrm{X}$ & $\ldots$ & $1.10(0.12)$ & $0.69(0.03)$ & $-0.1020(0.0570)$ & $\ldots$ & 7.04 & 1.01 \\
\hline 07af & $\ldots$ & $1.20(0.05)$ & $0.65(0.03)$ & $-0.0400(0.0520)$ & $\ldots$ & 2.04 & 0.94 \\
\hline 07au & $\ldots$ & $1.95(0.11)$ & $0.94(0.08)$ & $1.0840(0.0580)$ & $\ldots$ & 5.88 & 0.97 \\
\hline $07 b c$ & $\ldots$ & $1.35(0.07)$ & $0.67(0.04)$ & $0.2850(0.0900)$ & $\ldots$ & 2.92 & 0.91 \\
\hline 07bd & $\ldots$ & $\ldots$ & $\ldots$ & $0.2900(0.0970)$ & $\ldots$ & 3.93 & 3.93 \\
\hline $07 \mathrm{ca}$ & $\ldots$ & $\ldots$ & $\ldots$ & $-0.2140(0.0570)$ & $\ldots$ & 2.92 & 2.93 \\
\hline $07 \mathrm{ci}$ & $\ldots$ & $\ldots$ & $0.86(0.03)$ & $0.8830(0.0780)$ & $\ldots$ & $\ldots$ & 0.99 \\
\hline
\end{tabular}


Table 9

(Continued)

\begin{tabular}{lcccrcc}
\hline \hline SN & $\Delta m_{15}(U)$ & $\Delta m_{15}(B)$ & $\Delta m_{15}(V)$ & $\Delta$ & $\Delta t(U)$ & $\Delta t(B)$ \\
\hline $07 \mathrm{co}$ & $1.28(0.22)$ & $1.14(0.09)$ & $0.70(0.07)$ & $-0.0410(0.0780)$ & 2.69 & 1.16 \\
07cq & $\ldots$ & $1.17(0.18)$ & $0.61(0.07)$ & $0.0520(0.0710)$ & $\ldots$ & 0.85 \\
07F & $\ldots$ & $0.93(0.07)$ & $0.58(0.06)$ & $-0.1400(0.0510)$ & $\ldots$ & 3.84 \\
07qe & $\ldots$ & $0.98(0.05)$ & $0.59(0.06)$ & $-0.2570(0.0490)$ & $\ldots$ & 1.01 \\
07S & $\ldots$ & $0.88(0.08)$ & $0.62(0.08)$ & $-0.3230(0.0400)$ & $\ldots$ & 3.84 \\
08bf & $\ldots$ & $1.01(0.09)$ & $0.59(0.08)$ & $-0.1790(0.0640)$ & 2.06 & 4.83 \\
\end{tabular}

Notes. $\Delta t$ is the time between the closest point before and the closest point after maximum light. $\Delta$ is the light-curve shape parameter from MLCS2k2 with $R_{V}=1.7$.

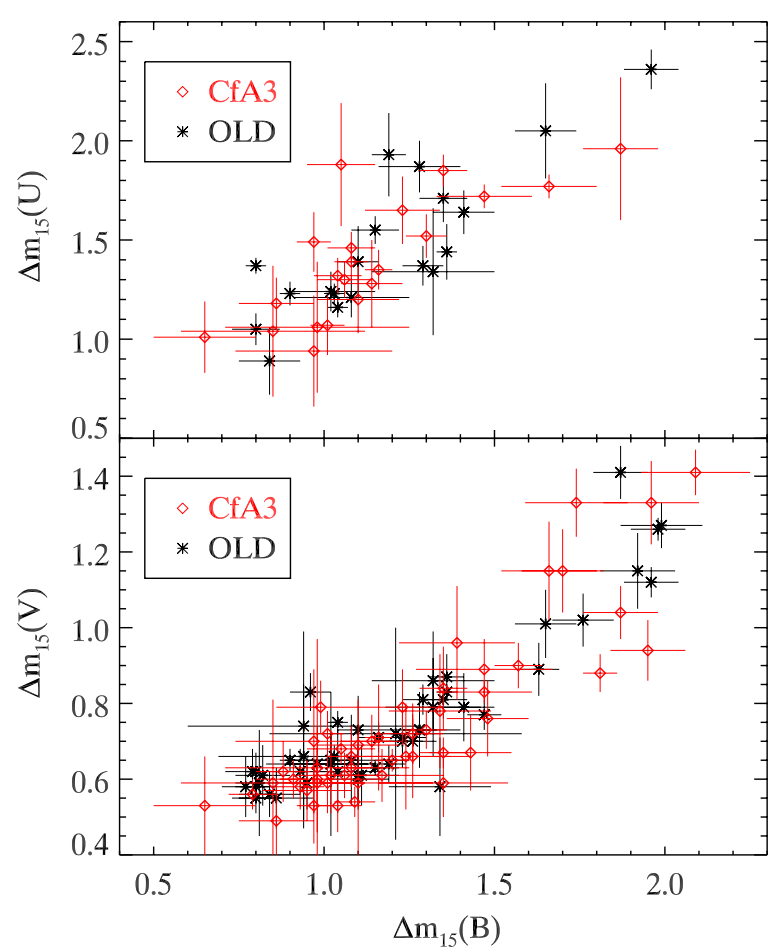

Figure 15. Plots of $\Delta m_{15}(U)$ and $\Delta m_{15}(V)$ vs. $\Delta m_{15}(B)$, measured directly from suitable SN Ia light curves. A linear correlation is seen in the $U$ and $B$ data. A tight correlation exists in $B$ and $V$ between the slow and normal decliners while the faster decliners (many of which are 1991bg-like objects) show larger scatter. (A color version of this figure is available in the online journal.)

A histogram of $A_{V}$, as measured by MLCS2k2, is shown in Figure 20. There are 133 CfA3 and 70 OLD objects, all with $z_{\mathrm{CMB}} \geqslant 0.01$ and with good MLCS2k2 fits. Their distributions are quite similar-the KS test gives a probability of $74 \%$ that they are from the same distribution.

Finally, in Figure 21, a histogram of $\Delta$ for the two samples is shown. In part, due to our prioritization of fast and slow decliners in our observing strategy, CfA3 has a wider distribution, most noticeably in the most-negative end and in the range around $\Delta=0.2$. The increased number of highly negative $\Delta \mathrm{SN}$ Ia is especially helpful for dark energy studies where these brighter objects are preferentially found due to magnitudelimited high- $z$ searches. In H09, the three high-redshift samples used (ESSENCE, SNLS, and Higher-Z) do not have any objects with $\Delta>0.75$. The KS test gives a probability of $9.4 \%$ that the two samples are from the same distribution. This should not be interpreted as an intrinsic difference in the underlying populations from which the CfA3 and OLD samples were drawn but, rather, as evidence of different selection effects, mostly

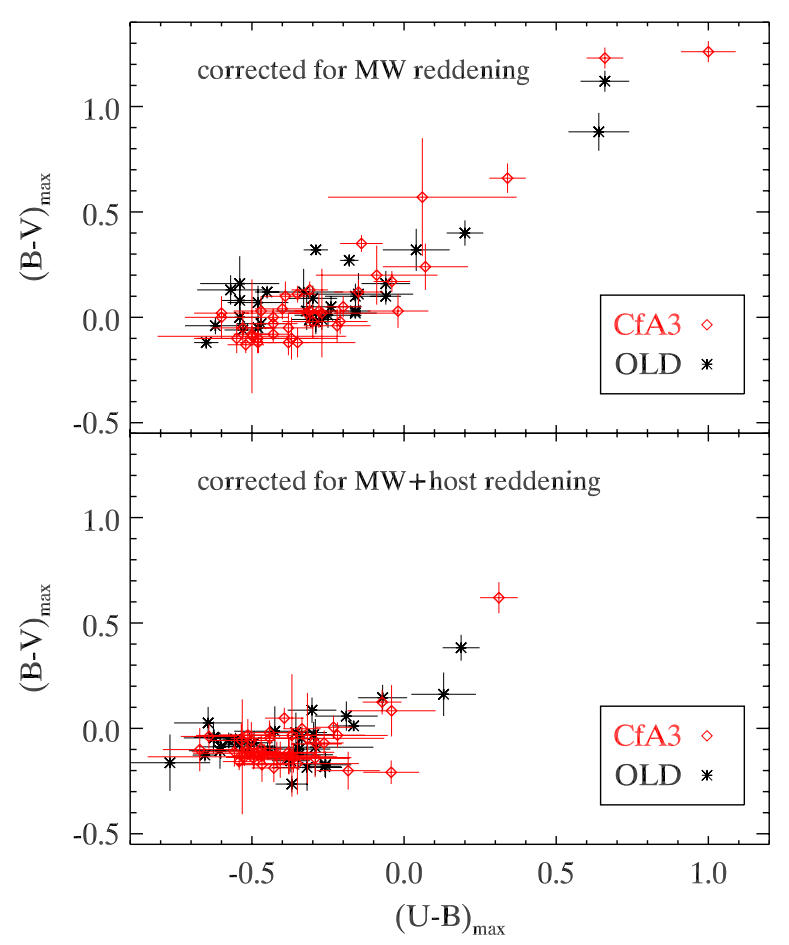

Figure 16. Peak SN Ia color, before and after MLCS2k2 $\left(R_{V}=1.7\right)$ correction for host reddening. Milky Way reddening is removed from both panels. The bottom panel gives a good idea of intrinsic $B-V$ and $U-B$ color at $B$ maximum The three reddest objects in the bottom panel are, in the order of increasing redness: SN 1986G, 1999by, and 2005ke.

(A color version of this figure is available in the online journal.)

related to which objects were chosen to be observed. Of the 133 CfA3 and 70 OLD SNe Ia at $z_{\mathrm{CMB}} \geqslant 0.01,42$ CfA3 and 28 OLD SNe Ia have $\Delta m_{15}(B)$ values and they are more similar in their light-curve shape distributions, producing KS-test probabilities of $70 \%$ for arising from the same $\Delta m_{15}(B)$ population and $53 \%$ for arising from the same $\Delta$ population. An exact match in $\Delta m_{15}(B)$ and $\Delta$ KS probabilities should not be expected since $\Delta m_{15}(B)$ and $\Delta$ are not perfectly correlated.

\subsection{Intrinsic Absolute Magnitude}

Using a lambda cold dark matter $(\Lambda \mathrm{CDM})$ universe with $\left(\Omega_{m}=0.3, \Omega_{\Lambda}=0.7, h=0.7\right)$, we calculate distance moduli based on the CMB redshifts for all the CfA3 and OLD $\mathrm{SNe}$ Ia with $z_{\mathrm{CMB}} \geqslant 0.01$ and good MLCS2k2 $\left(R_{V}=1.7\right)$ fits for which we could also directly measure the peak $U B V$ magnitudes. We subtract off the distance moduli in the top panels of Figures 22-24 to show the absolute magnitudes before correction for host-galaxy extinction. In the bottom panels, we also subtract off the MLCS2k2-calculated host extinction to 


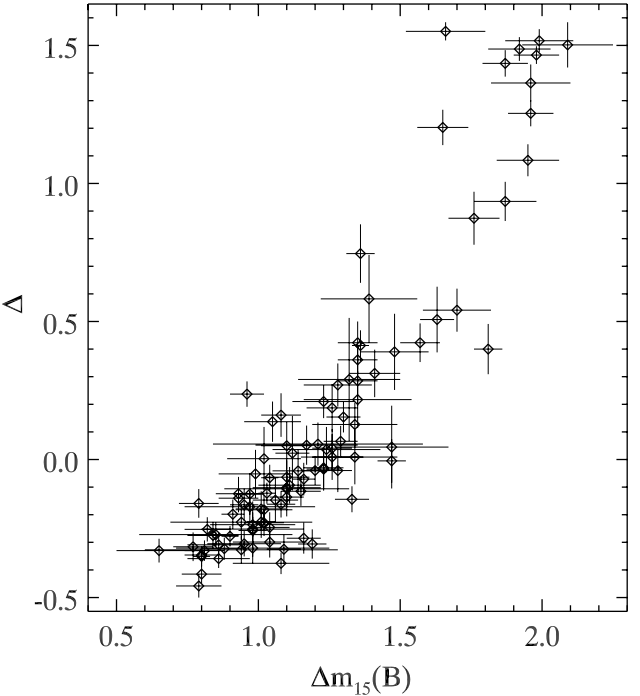

Figure 17. MLCS2k $2 \Delta$ vs. $\Delta m_{15}(B)$. Fairly good correlation between the two except at the largest values of $\Delta m_{15}(B)$, where many of the objects are $1991 \mathrm{bg}$ like.

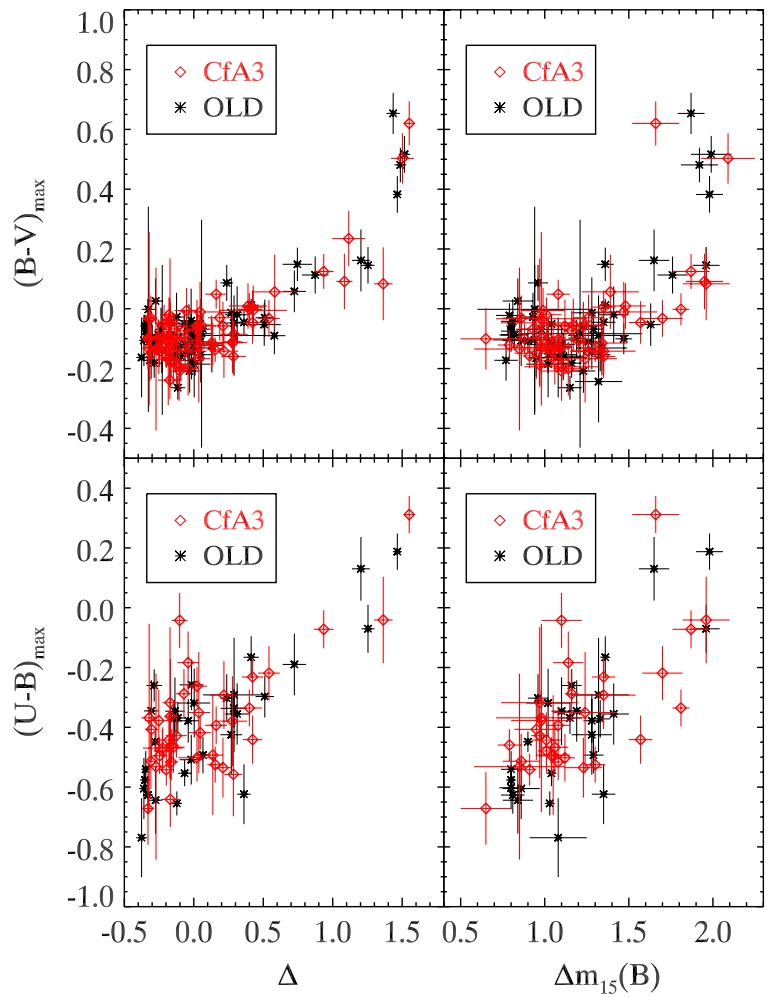

Figure 18. $B-V$ and $U-B$ peak colors, corrected for MLCS2k2-calculated reddening is shown for all well measured objects, with no cut on redshift. The upper-left panel shows an upward curving distribution that can be broken into three, somewhat arbitrary, groupings. The slow and normal decliners with $-0.4 \leqslant \Delta<0.7$ have a typical color of $(B-V)_{\max } \approx-0.1$, although the upward trend in color starts in the right portion of this group. The second group has a typical color of 0.1 with $-0.7<\Delta<1.4$ and includes objects similar to both 1992A and 1986G. This second group may be a "transitional" group (or "intersectional" if there are two underlying groups), both photometrically and spectroscopically, to the third group, consisting of $1991 \mathrm{bg}$-like SN Ia, with $(B-V)_{\max } \approx 0.5$ and $\Delta \approx 1.5$.

(A color version of this figure is available in the online journal.)

give a good estimate of the intrinsic absolute magnitudes of our sample.

The two most noticeable things are that the extinction correction does a good job of reducing the scatter and that $\Delta$ is

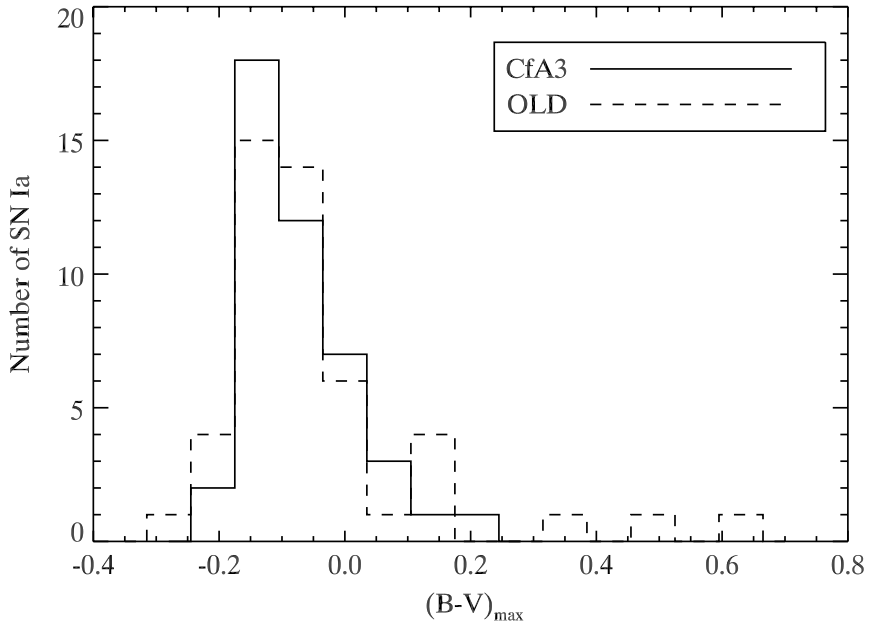

Figure 19. 44 CfA3 and 48 OLD SNe Ia with $z_{\mathrm{CMB}} \geqslant 0.01$ had reliable, direct $(B-V)_{\max }$ measurements. The two samples show excellent agreement-a KS test gives $87 \%$ probability that the two samples are drawn from the same distribution.

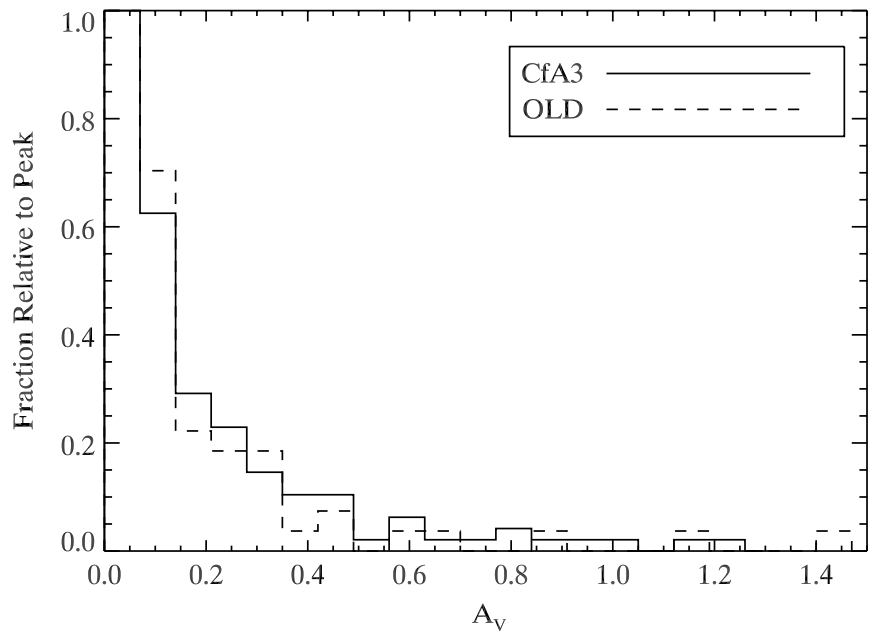

Figure 20. Histograms of the CfA 3 and OLD SN Ia $V$ extinction as calculated by MLCS2k2 $\left(R_{V}=1.7\right) .133$ CfA3 and 70 OLD, useful for cosmological measurements, with $z_{\mathrm{CMB}} \geqslant 0.01$ and good MLCS2k2 fits are included. The distributions are normalized to their respective peaks and good agreement is seen-a KS test gives $74 \%$ probability that the two samples are drawn from the same distribution.

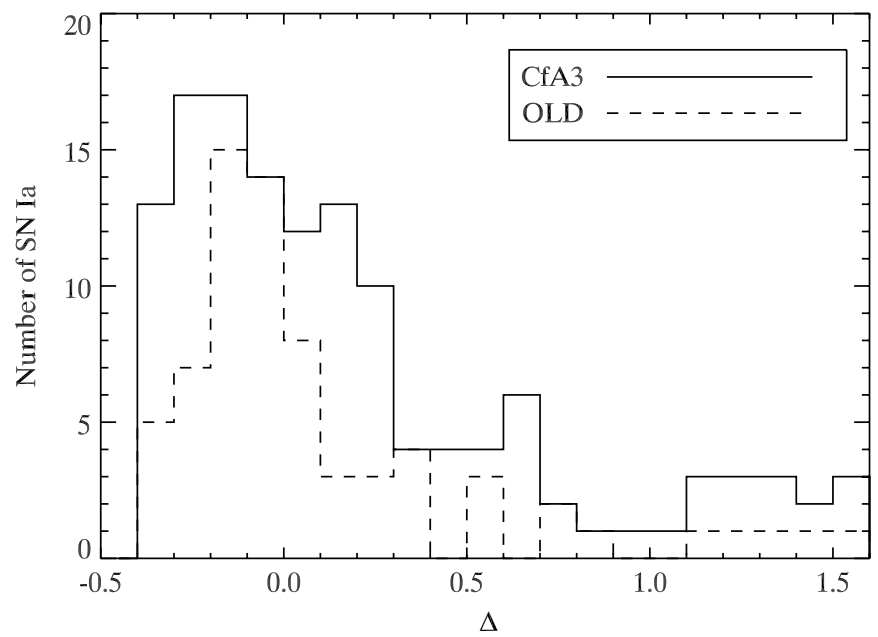

Figure 21. Histograms of the $133 \mathrm{CfA} 3$ and 70 OLD SN Ia values of $\Delta$, all at $z_{\mathrm{CMB}} \geqslant 0.01$. The CfA3 sample shows a wider distribution in $\Delta$, probably due to our prioritization of slow and fast decliners. 


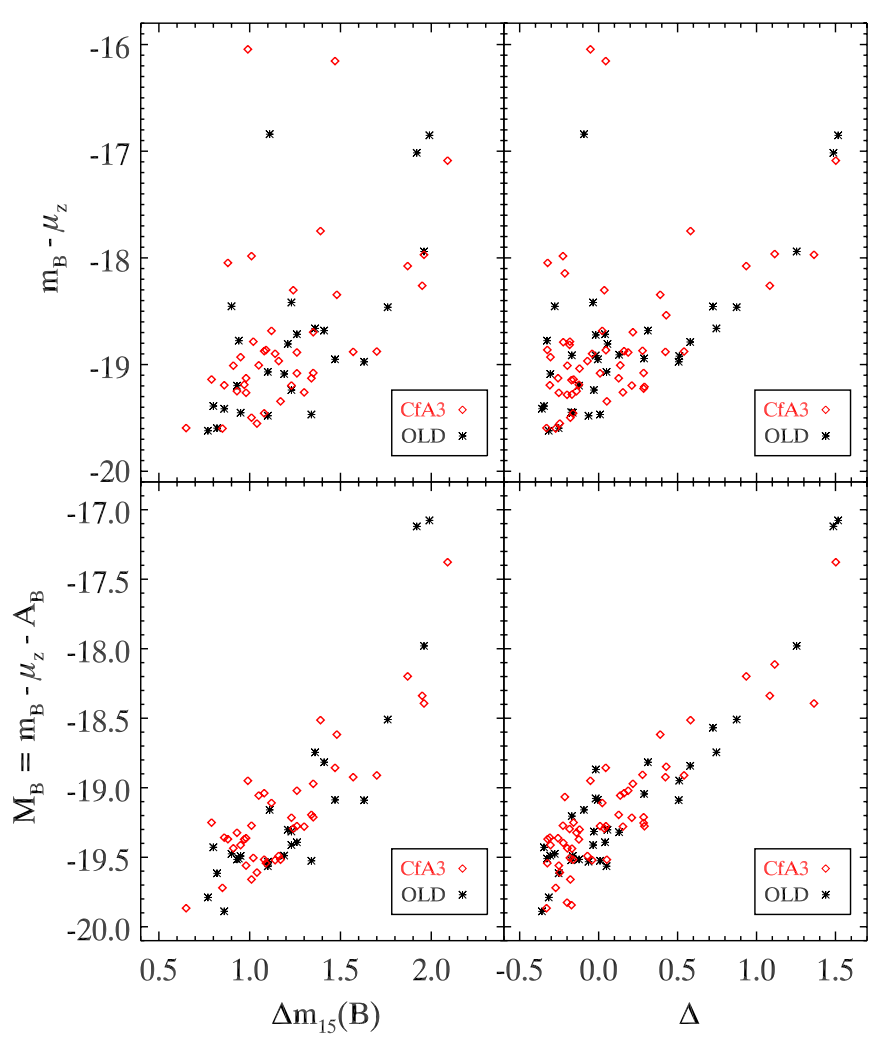

Figure 22. SN Ia absolute magnitude vs. $\Delta m_{15}(B)$ and $\Delta$. The top panels show SN Ia peak apparent magnitude in $B$, directly measured from the suitable light curves, after $K$-corrections and correction for MW extinction, minus the distance modulus $\left(\Omega_{M}=0.3, \Omega_{\Lambda}=0.7, h=0.7\right)$. All objects are at $z_{\mathrm{CMB}} \geqslant 0.01$ and error bars are omitted to not obscure the data points. The lower panels further subtract off the host-galaxy extinction, $A_{B}$, as calculated by MLCS2k2 $\left(R_{V}=1.7\right)$, giving a good estimate of SN Ia intrinsic absolute magnitude, $M_{B}$. This is plotted against $\Delta m_{15}(B)$ and $\Delta$. A linear trend is evident in both lower panels, except for the faintest objects which are all 1991bg-like. The relation between $M_{B}$ and $\Delta$ is tighter than between $M_{B}$ and $\Delta m_{15}(B)$. If objects below $z_{\mathrm{CMB}}=0.01$ were included then three more $1991 \mathrm{bg}$-like SNe Ia would be in the vicinity of $\left(\Delta=1.5, M_{B}=-17\right)$, but with higher uncertainty due to peculiar velocities.

(A color version of this figure is available in the online journal.)

more tightly correlated with intrinsic absolute magnitude than is $\Delta m_{15}(B)$. Part of this may be due to the larger uncertainties in our $\Delta m_{15}(B)$ measurements.

Focusing on the lower right panel of Figure 22, there appears to be a linear relation between $B$-band intrinsic absolute magnitude and $\Delta$, out to $\Delta \approx 1.2$. In $V$, the three faint and high- $\Delta$ objects are $1991 \mathrm{bg}$-like objects. We also note that if the $z=0.01$ cut were not made that there would be several more $1991 \mathrm{bg}$-like objects from our direct light-curve measurements with $\Delta \approx 1.5$ and $M_{B} \approx-17$, confirming the faint and separate nature of $1991 \mathrm{bg}$-like SNe Ia. In order to include these, MLCS2k2 uses a positive term in $\Delta$ but this comes at the expense of underestimating the luminosity of the SN Ia in the range $0.7<\Delta<1.2$ (see Figure 23 where the solid line shows the dependence of intrinsic absolute magnitude versus $\Delta$ for the MLCS2k2 model light curves). It may be better to avoid 1991bg-like objects altogether for use in the light-curve-shape/magnitude relation, or to at least treat them separately. Removing them can be accomplished by spectroscopic identification of $1991 \mathrm{bg}$-like features or by simply removing all objects above a certain $\Delta$ and/or intrinsic peak color.

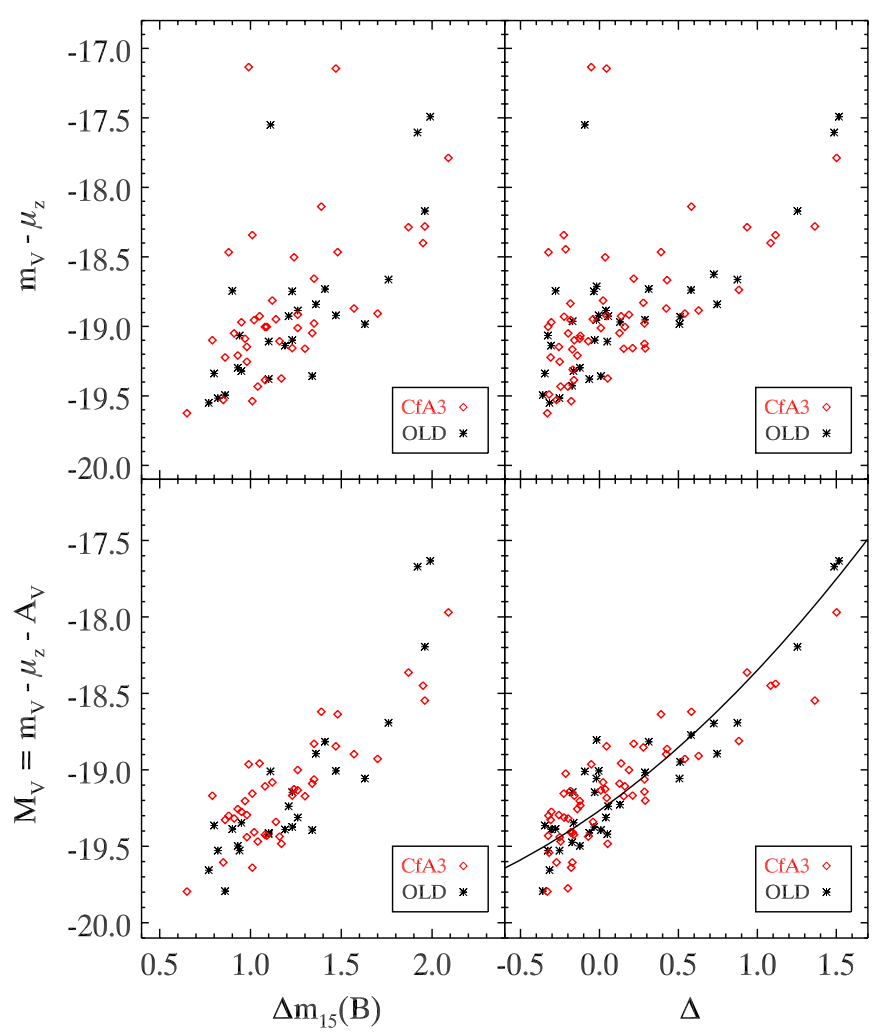

Figure 23. SN Ia $V$ absolute magnitude vs. $\Delta m_{15}(B)$ and $\Delta$. Same as in Figure 22 but for $V$. The solid line is the MLCS2k2 model intrinsic absolute magnitude, $M_{V}(\Delta)$, from Jha et al. (2007).

(A color version of this figure is available in the online journal.)

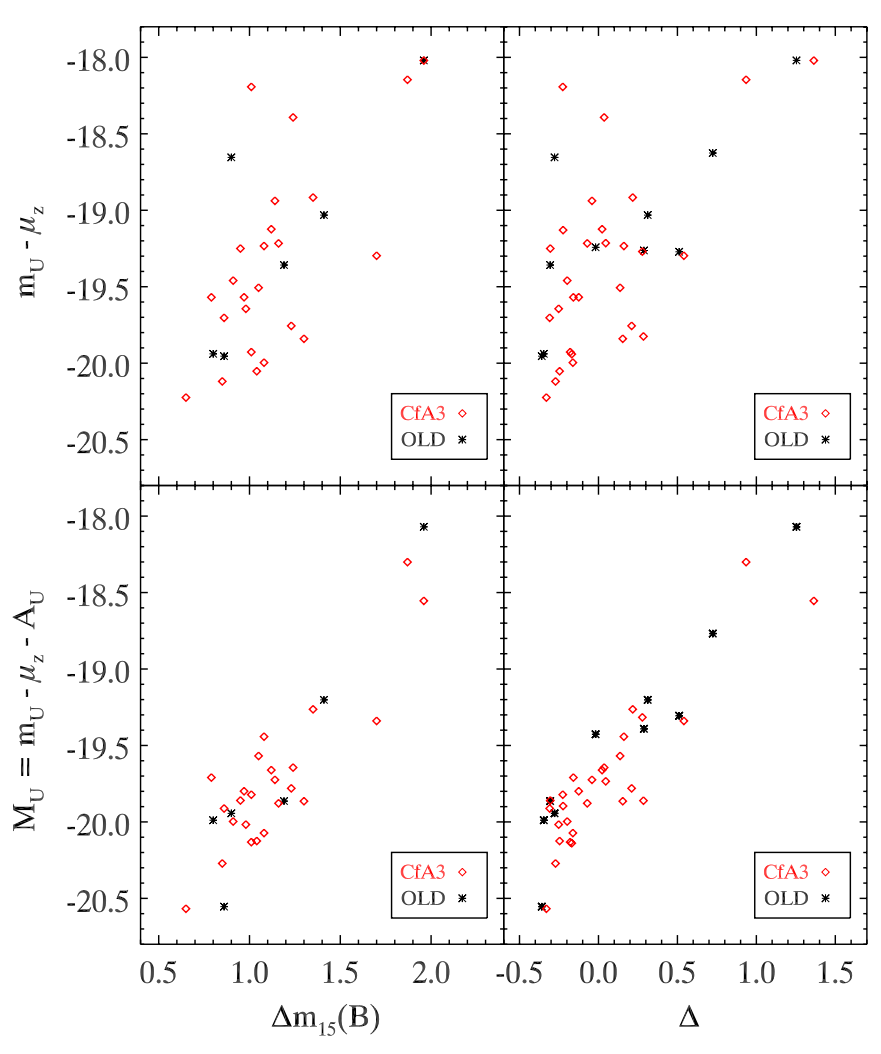

Figure 24. SN Ia $U$ absolute magnitude vs. $\Delta m_{15}(B)$ and $\Delta$. Same as in Figure 22 but for $U$. There is fairly good correlation between $M_{U}$ and light-curve shape. (A color version of this figure is available in the online journal.) 


\section{CONCLUSION}

The goal of our research was to produce a large sample of nearby SN Ia light curves that would better sample the whole range of SN Ia and serve to reduce the statistical and systematic uncertainties in dark energy calculations using $\mathrm{SNe}$ Ia.

We have presented 185 nearby CfA3 SN Ia light curves, adding a significant number of fast and slow decliners. We have shown that the photometry is internally consistent, and that it is externally consistent at roughly the same level as seen in other nearby SN Ia photometry. The intrinsic properties of $\mathrm{SNe}$ Ia have been explored in $U B V$, confirming previous studies. One of the most important findings is that $(B-V)_{\max }$ and the peak intrinsic magnitude in $B$ and $V$ do not correlate well with light-curve shape among the fast decliners (see Figures 18, 22, and 23). However, when the $1991 \mathrm{bg}$-like objects are removed, the remaining fast decliners still seem to be well correlated with color and intrinsic absolute magnitude. This suggests that 1991bg-like SN Ia should be excluded from light-curve/distance fitter training samples and from dark energy studies. We believe that this will improve the performance of fitters, like MLCS2k2, that have used them in their training samples.

The CfA3 sample is an important contribution to dark energy studies because it is the largest homogeneously reduced nearby sample, doubling the cosmologically useful sample. The addition of the CfA3 sample to the literature SN Ia and its effects on the dark energy calculations are explored in H09. H09 show that the statistical uncertainty in $w$ is reduced by a factor of $1.2-1.3$ by adding the CfA3 sample. The CfA3 sample lowers the statistical uncertainty on static dark energy measurements to the point where systematic uncertainties begin to dominate. The CfA3 sample can be used to lower systematic uncertainties in dark energy studies in two ways. First, either the complete sample or the CfA3-Keplercam subsample can be used as a stand-alone nearby sample that reduces or eliminates the uncertainties that arise from different reduction pipelines, filters, cameras, and comparison-star calibration among nearby SNe Ia. Second, it will be very useful for retraining light-curve fitters and making them more precise. The two CfA3 subsamples, CfA3Keplercam and CfA3-4Shooter, are the largest and secondlargest homogeneously observed and reduced nearby samples to date. A large fraction of the CfA3 objects have spectra. A few dozen CfA 3 objects also have near-infrared photometry and will help disentangle host-galaxy reddening from intrinsic SN Ia color. This will lead to a large decrease in the systematic uncertainty associated with host-galaxy dust absorption. Other large optical samples will soon be published too, with dozens of objects overlapping the CfA3 sample, providing a good opportunity to search for systematic errors and better combine data sets from different groups. We plan on publishing another 70-100 SN Ia light curves (CfA4 sample) when the hostgalaxy reference images are obtained and the comparison star calibration is completed. These have been observed and reduced in the same way as the CfA3-Keplercam subsample and when added together will number roughly 200.

Future nearby SN Ia studies should focus on reducing their statistical and systematic photometric uncertainties by obtaining higher S/N measurements and improved understanding of their passbands and SN Ia calibration. The goal of $1 \%$ SN Ia photometry should be pursued so that more precise SN Ia measurements can produce tighter limits on both static and especially dynamic models of dark energy. The largest hurdles to achieving $1 \%$ photometry are in understanding atmospheric transmission and instrumental passbands. Efforts along the lines of Stubbs \& Tonry (2006) should be pursued and improved. Use of calibrated photodiodes and monochromatic light allows for a precise determination of the system's transmission function. The pixel-by-pixel CCD response can be precisely determined by taking monochromatic flat fields at a sufficiently sampled range of wavelengths and measuring the intensity of this light with the calibrated photodiode. Additionally, improved treatment of host-galaxy contamination of SN light should be developed, through improved image-subtraction software and/ or improved galaxy+SN models that measure both without image subtraction.

We thank the staff at FLWO for their dedicated work in maintaining the $1.2 \mathrm{~m}$ telescope and instruments. This work has been supported, in part, by NSF grant AST0606772 to Harvard University. A.R. thanks the Goldberg Fellowship Program for its support.

Facility: FLWO: $1.2 \mathrm{~m}$

\section{REFERENCES}

Alard, C. 2000, A\&AS, 144, 363

Alard, C., \& Lupton, R. 1998, ApJ, 503, 325

Aldering, G., et al. 2002, Proc. SPIE, 4836, 61

Anupama, G. C., et al. 2005, A\&A, 429, 667

Becker, A. C., et al. 2004, ApJ, 611, 418

Benetti, S., et al. 2004, MNRAS, 348, 261

Bertin, E., et al. 2002, in Proc. ASP Conf. Ser. 281, Astronomical Data Analysis Software and Systems XI, ed. D. A. Bohlender, D. Durand, \& T. H. Handley (San Francisco, CA: ASP), 228

Bessell, M. S. 1990, PASP, 102, 1181

Conley, A., et al. 2007, ApJ, 664, L13

Dilday, B., et al. 2008, ApJ, 682, 262

Fabricant, D., et al. 1998, PASP, 110, 79

Frieman, J. A., et al. 2008, AJ, 135, 338

Garg, A., et al. 2007, AJ, 133, 403

Garnavich, P. M., et al. 2004, ApJ, 613, 1120

Goldhaber, G., et al. 2001, ApJ, 558, 359

Guy, J., et al. 2005, A\&A, 443, 781

Guy, J., et al. 2007, A\&A, 466, 11

Hamuy, M., et al. 1996a, AJ, 112, 2391

Hamuy, M., et al. 1996b, AJ, 112, 2408

Hamuy, M., et al. 2006, PASP, 118, 2

Haugbolle, T., et al. 2007, ApJ, 661, 650

Hicken, M., et al. 2007, ApJ, 669, L17

Hicken, M., et al. 2009, ApJ, in press

Jha, S., Riess, A. G., \& Kirshner, R. P. 2007, ApJ, 659, 122

Jha, S., et al. 2006, AJ, 131, 527

Kowal, C. T. 1968, AJ, 73, 1021

Kowalski, M., et al. 2008, ApJ, 686, 749

Krisciunas, K., et al. 2000, ApJ, 539, 658

Krisciunas, K., et al. 2001, AJ, 122, 1616

Krisciunas, K., et al. 2003, AJ, 125, 166

Krisciunas, K., et al. 2004a, AJ, 127, 1664

Krisciunas, K., et al. 2004b, AJ, 128, 3034

Krisciunas, K., et al. 2006, AJ, 131, 1639

Landolt, A. U. 1992, AJ, 104, 372

Leonard, D. C., et al. 2005, ApJ, 632, 450

Li, W., et al. 2006, ApJ, 641, 1060

Maeda, K., et al. 2009, MNRAS, 394, 239

Mandel, K., et al. 2009, ApJ, submitted

Matheson, T., et al. 2008, AJ, 135, 1598

Miknaitis, G., et al. 2007, ApJ, 666, 674

Monet, D., et al. 1998, USNO-A2.0 (11 CD-ROMs; Flagstaff, AZ: U.S. Naval Observatory)

Monet, D., et al. 2003, AJ, 125, 984

Neill, J. D., Hudson, M. J., \& Conley, A. 2007, ApJ, 661, L123

Norgaard-Nielsen, H. U., et al. 1989, Nature, 339, 523

Perlmutter, S., et al. 1999, ApJ, 517, 565 
Phillips, M. M. 1993, ApJ, 413, L105

Phillips, M. M., et al. 2007, PASP, 119, 360

Rest, A., et al. 2005, ApJ, 634, 1103

Riess, A. G., Press, W. H., \& Kirshner, R. P. 1995, ApJ, 445, L91

Riess, A. G., Press, W. H., \& Kirshner, R. P. 1996, ApJ, 473, 88

Riess, A. G., et al. 1998, AJ, 116, 1009

Riess, A. G., et al. 1999, AJ, 11, 707

Sako, M., et al. 2005, arXiv:astro-ph/0504455v2

Schechter, P. L., Mateo, M., \& Saha, A. 1993, PASP, 105, 1342

Schlegel, D. J., Finkbeiner, D. P., \& Davis, M. 1998, ApJ, 500, 525

Smith, J. A., et al. 2002, AJ, 123, 2121

Stanishev, V., et al. 2007, A\&A, 469, 645
Stritzinger, M., et al. 2002, AJ, 124, 2100

Stubbs, C. W., \& Tonry, J. L. 2006, ApJ, 646, 1436

Suntzeff, N. B. 2000, in Cosmic Explosions, Tenth Astrophysics Conf., ed. S. S., Holt \& W. W. Zhang (New York: AIP), 65

Tammann, G. A., \& Leibundgut, B. 1990, A\&A, 236, 9

Tody, D. 1993, in ASP Conf. Ser. 52, in Astronomical Data Analysis Software and Systems II, IRAF in the Nineties, ed. R. J. Hanisch, R. J. V. Brissenden, \& J. Barnes (San Francisco, CA: ASP), 173

van Dokkum, P. G. 2001, PASP, 113, 1420

Wang, X., et al. 2008, ApJ, 677, 1060

Wang, X., et al. 2009, ApJ, 697, 380

Wood-Vasey, W. M., et al. 2008, ApJ, 689, 377

Zacharias, N., et al. 2004, AJ, 127, 3043 